JOURNAL OF THE

AMERICAN MATHEMATICAL SOCIETY

Volume 22, Number 1, January 2009, Pages 233-286

S 0894-0347(08)00612-7

Article electronically published on September 3, 2008

\title{
CONSTRUCTIBLE SHEAVES AND THE FUKAYA CATEGORY
}

\author{
DAVID NADLER AND ERIC ZASLOW
}

\section{CONTEnts}

1. Introduction

1.1. Microlocal geometry 235

1.2. Summary 236

1.3. Mirror symmetry 239

2. $A_{\infty}$-categories 240

2.1. Preliminaries 240

2.2. $A_{\infty}$-modules 240

2.3. Triangulated $A_{\infty}$-categories $\quad 241$

2.4. Twisted complexes 241

2.5. Homological perturbation theory $\quad 242$

3. Analytic-geometric categories $\quad 242$

3.1. Basic definitions 243

3.2. Background results 243

4. Constructible sheaves 245

4.1. Standard objects 245

4.2. Standard triangles 245

4.3. Standard objects generate 246

4.4. Open submanifolds 247

4.5. Smooth boundaries 248

4.6. Morse theory 250

5. The Fukaya category 255

5.1. Basics of $T^{*} X \quad 256$

5.2. Lagrangians 258

5.3. Brane structures $\quad 261$

5.4. Definition of Fukaya category $\quad 263$

6. Embedding of standard objects $\quad 268$

6.1. Preliminaries 268

6.2. Variable dilation $\quad 269$

6.3. Separation $\quad 270$

6.4. Perturbations 272

6.5. Relation to Morse theory 275

7. Arbitrary standard objects 278

7.1. Submanifold category 279

7.2. Morse theory interpretation 281

Received by the editors October 5, 2006.

2000 Mathematics Subject Classification. Primary 53D40, 32S60.

(C)2008 American Mathematical Society Reverts to public domain 28 years from publication 
7.3. Identification with standard branes $\quad 282$

7.4. Other objects 284

Acknowledgments $\quad 284$

References $\quad 285$

\section{INTRODUCTION}

In this paper, we study the relationship between two natural invariants of a real analytic manifold $X$. The first is the Fukaya category of Lagrangian submanifolds of the cotangent bundle $T^{*} X$. The second is the derived category of constructible sheaves on $X$ itself. The two are naively related by the theory of linear differential equations - that is, the study of modules over the ring $\mathcal{D}_{X}$ of differential operators on $X$. On the one hand, Lagrangian cycles in $T^{*} X$ play a prominent role in the microlocal theory of $\mathcal{D}_{X}$-modules. On the other hand, in the complex setting, the Riemann-Hilbert correspondence identifies regular, holonomic $\mathcal{D}_{X}$-modules with constructible sheaves. In what follows, we give a very brief account of what we mean by the Fukaya category of $T^{*} X$ and the constructible derived category of $X$, and then state our main result.

Roughly speaking, the Fukaya category of a symplectic manifold is a category whose objects are Lagrangian submanifolds and whose morphisms and compositions are built out of the quantum intersection theory of Lagrangians. This is encoded by the moduli space of pseudoholomorphic maps from polygons with prescribed Lagrangian boundary conditions. Since $T^{*} X$ is noncompact, there are many choices to be made as to which Lagrangians to allow and how to obtain well-behaved moduli spaces of pseudoholomorphic maps. One approach is to insist that the Lagrangians are compact. With this assumption, the theory is no more difficult than that of a compact symplectic manifold. One perturbs the Lagrangians so that their intersections are transverse, and then convexity arguments guarantee compact moduli spaces.

Our version of the Fukaya category $F u k\left(T^{*} X\right)$ includes both compact and noncompact exact Lagrangians. We work with exact Lagrangians that have well-defined limits at infinity. To make this precise, we consider a compactification of $T^{*} X$ and assume that the closures of our Lagrangians are subanalytic subsets of the compactification. Two crucial geometric statements follow from this assumption. First, the boundaries of our Lagrangians are Legendrian subvarieties of the divisor at infinity. Second, for any metric on the fibers of $T^{*} X$, its restriction to one of our Lagrangians has no critical points near infinity. These facts allow us to make sense of "intersections at infinity" by restricting our perturbations to those which are normalized geodesic flow near infinity for carefully prescribed times. Given suitable further perturbations (which are available in intended applications), we then obtain compact moduli spaces of pseudoholomorphic maps. The resulting Fukaya category $F u k\left(T^{*} X\right)$ has many of the usual properties that one expects from both a topological and categorical perspective.

The second invariant of the real analytic manifold $X$ which we consider is the derived category $D_{c}(X)$ of constructible sheaves on $X$ itself. This is a triangulated category which encodes the topology of subanalytic subsets of $X$. To give a sense of the size of $D_{c}(X)$, its Grothendieck group is the group of constructible functions on 
$X$ - that is, functions which are constant along some subanalytic stratification, for example a triangulation. Examples of objects of $D_{c}(X)$ include closed submanifolds equipped with flat vector bundles. More generally, we have the so-called standard and costandard objects associated to a locally closed submanifold $Y \subset X$ equipped with a flat vector bundle $\mathcal{E}$. Informally, one may think of the standard object as the complex of singular cochains on $Y$ with values in $\mathcal{E}$, and the costandard as the complex of relative singular cochains on $(Y, \partial Y)$ with values in $\mathcal{E}$. A key observation is that morphisms between these objects are naturally the singular cohomology of certain subsets of $X$ with values in flat vector bundles.

One formulation of our main result is the following. As we outline below, it may be viewed as a categorification of the characteristic cycle construction.

Theorem 1.0.1. Let $X$ be a real analytic manifold. Then there is a canonical embedding of derived categories

$$
D_{c}(X) \hookrightarrow D F u k\left(T^{*} X\right) .
$$

The result reflects an underlying quasi-embedding of $\mathrm{dg}$ and $A_{\infty}$-categories. Further arguments show that this is in fact a quasi-equivalence [28].

The remainder of the Introduction is divided into several parts. In the section immediately following, we discuss motivations for our main result from the longdeveloping theory of microlocal geometry. In the section after that, we explain the general outline of the proof of our main result. Finally, we speculate on possible applications in the context of mirror symmetry.

1.1. Microlocal geometry. The main result of this paper has a natural place in the context of microlocal geometry. Broadly speaking, sheaf theory on a real analytic manifold $X$ may be thought of as a tool to understand local analytic and topological phenomena and how they assemble into global phenomena. Many aspects of the theory are best understood from a microlocal perspective, or in other words as local phenomona on the cotangent bundle $T^{*} X$. Here we collect a short account of some results from this subject that naturally point toward our main result. What we present is not intended to be an exhaustive overview of the subject. For that we refer the reader to the book of Kashiwara-Schapira [20. It contains many original results, presents a detailed development of the subject, and includes historical notes and a comprehensive bibliography.

Our main result may be viewed as a categorification of the characteristic cycle construction for real constructible sheaves introduced by Kashiwara [17]. (For foundational material on microlocal constructions such as the singular support, see Kashiwara-Schapira [19].) Given a constructible complex of sheaves $\mathcal{F}$ on $X$, its characteristic cycle $C C(\mathcal{F})$ is a conical Lagrangian cycle in $T^{*} X$ (with values in the pullback of the orientation sheaf of $X$ ) which encodes the singularities of the original complex. The multiplicity of $C C(\mathcal{F})$ at a given covector is the Euler characteristic of the local Morse groups of the complex with respect to the covector. If a covector is not in the support of $C C(\mathcal{F})$, it means that there is no obstruction to propagating local sections of $\mathcal{F}$ in the direction of the covector. So for example, the characteristic cycle of a flat vector bundle on $X$ is the zero section in $T^{*} X$ with multiplicity the dimension of the vector bundle. More generally, the characteristic cycle of a flat vector bundle on a closed submanifold is the conormal bundle to the submanifold with multiplicity the dimension of the vector bundle. 
As mentioned earlier, the Grothendieck group of the constructible derived category $D_{c}(X)$ is the space of constructible functions on $X$. The characteristic cycle construction descends to an isomorphism from constructible functions to the group of conical Lagrangian cycles in $T^{*} X$. From this vantage point, there are many results that might lead one to our main result. First, there is the index formula of Dubson [6] and Kashiwara [17. This states that given a constructible complex of sheaves $\mathcal{F}$, its Euler characteristic $\chi(X, \mathcal{F})$ is equal to the intersection of Lagrangian cycles $C C(\mathcal{F}) \cdot[d f]$ where $d f$ is the graph of a sufficiently generic function $f: X \rightarrow \mathbb{R}$. More generally, given two constructible complexes of sheaves $\mathcal{F}_{1}, \mathcal{F}_{2}$, a formula of MacPherson (see the introduction of [11, the lecture notes [13, and a Floer-theoretic interpretation [21, 22]) expresses the Euler characteristic of their tensor product in terms of the intersection of their characteristic cycles:

$$
\chi\left(X, \mathcal{F}_{1} \stackrel{L}{\otimes} \mathcal{F}_{2}\right)=C C\left(\mathcal{F}_{1}\right) \cdot C C\left(\mathcal{F}_{2}\right) .
$$

The most direct influence on our main result is the work of Ginsburg [11] (in the complex affine case) and Schmid-Vilonen 32 (in general) on the functoriality of the characteristic cycle construction. Thanks to their work, one knows how to calculate the characteristic cycle $C C\left(R i_{*} \mathcal{F}\right)$ of the direct image under an open embedding $i: U \hookrightarrow X$. (The functoriality of the characteristic cycle under the other standard operations is explained by Kashiwara-Schapira 20.) In the subanalytic context, given an open subset $i: U \hookrightarrow X$, one can always choose a defining function $m: X \rightarrow \mathbb{R}_{\geq 0}$ for the boundary $\partial U \subset X$. By definition, $m$ is a nonnegative function whose zero set is precisely $\partial U \subset X$. With such a function in hand, the formula for open embeddings is the limit of Lagrangian cycles

$$
C C\left(R i_{*} \mathcal{F}\right)=\lim _{\epsilon \rightarrow 0_{+}}\left(C C(\mathcal{F})+\epsilon \Gamma_{d \log m}\right),
$$

where $\Gamma_{d \log m} \subset T^{*} U$ is the graph of the differential and the sum is set-theoretic. The proof of our main result may be interpreted as a categorification of this formula. We explain this in the next section.

1.2. Summary. To relate the constructible derived category $D_{c}(X)$ to the Fukaya category $F u k\left(T^{*} X\right)$, we proceed in several steps, some topological and some categorical.

It is well-known that the usual notions of category theory are too restrictive a context for dealing with the geometry of moduli spaces of pseudoholomorphic maps. To be precise, $F u k\left(T^{*} X\right)$ is not a usual category but rather an $A_{\infty}$-category. Relations among compositions of morphisms are determined by the bubbling of pseudoholomorphic disks, and this is not associative but only homotopy associative. The $A_{\infty}$-category formalism is a means to organize these homotopies (and the homotopies between the homotopies, and so on). In particular, morphisms in an $A_{\infty}$-category are represented by chain complexes to provide some homotopic flexibility. When this is the only added wrinkle, so that compositions of such morphisms are in fact associative, one arrives at the special case of a differential graded (dg) category. To an $A_{\infty}$-category one can assign an ordinary (graded) category by taking the cohomology of its morphism complexes. This allows for the perspective that these notions only differ from that of an ordinary category by providing more homotopic flexibility. (We collect some of the basic notions of $A_{\infty}$-categories in Section 2 below.) 
The derived category $D_{c}(X)$ is the cohomology category of a dg category $\operatorname{Sh}(X)$ whose objects are constructible complexes of sheaves. The morphisms in $\operatorname{Sh}(X)$ are defined by starting with the naive definition of morphisms of complexes, and then passing to the dg quotient category where quasi-isomorphisms are invertible. Our first step in reaching $F u k\left(T^{*} X\right)$ is to observe that $S h(X)$ is generated by its full subcategory consisting of standard objects associated to open submanifolds. In the subanalytic context, given an open subset $U \subset X$, one can always choose a defining function $m: X \rightarrow \mathbb{R}_{\geq 0}$ for the complement $X \backslash U$. By definition, $m$ is a nonnegative function whose zero set is precisely $X \backslash U$. To keep track of the choice of such a function, we define a dg category $\operatorname{Open}(X)$ as follows. Its objects are pairs $(U, m)$, where $U \subset X$ is open and $m$ is as described. Its morphisms are given by complexes of relative de Rham forms and are naturally quasi-isomorphic with those for the corresponding standard objects of $S h(X)$. In the language of dg categories, one can say that $S h(X)$ is a triangulated envelope of $O p e n(X)$ and that $D_{c}(X)$ is the derived category of both $\operatorname{Sh}(X)$ and $\operatorname{Open}(X)$.

The aim of our remaining constructions is to embed the $A_{\infty}$-category $\operatorname{Open}(X)$ into the Fukaya $A_{\infty}$-category $F u k\left(T^{*} X\right)$. It is simple to say where this $A_{\infty}$-functor takes objects of $O p e n(X)$. To explain this, we introduce some notation in a slightly more general context. Given a submanifold $Y \subset X$ and a defining function $m$ : $X \rightarrow \mathbb{R}_{\geq 0}$ for the boundary $\partial Y \subset X$, set $f: X \backslash \partial Y \rightarrow \mathbb{R}$ to be the logarithm $f=\log m$, and define the standard Lagrangian $\left.L_{Y, f} \subset T^{*} X\right|_{Y} \subset T^{*} X$ to be the fiberwise sum

$$
L_{Y, f}=T_{Y}^{*} X+\left.\Gamma_{d f}\right|_{Y}
$$

where $\left.\Gamma_{d f} \subset T^{*} X\right|_{X \backslash \partial Y}$ denotes the graph of $d f$ and the sum is taken fiberwise in $\left.T^{*} X\right|_{Y}$. By construction, $L_{Y, f}$ depends only on the restriction of $m$ to $Y$. In particular, for an open subset $U \subset X$, we could also take $m$ to be a defining function for the complement $X \backslash U$. In this case, the definition simplifies so that $L_{U, f}$ is just the graph $\Gamma_{d f}$ over $U$.

Now given an object $(U, m)$ of $O p e n(X)$, where $U \subset X$ is open and $m: X \rightarrow \mathbb{R}_{\geq 0}$ is a defining function for $X \backslash U$, we send it to the standard Lagrangian $L_{U, f} \subset T^{*} X$, where $f: U \rightarrow \mathbb{R}$ is given by $f=\log m$. If $U$ is not all of $X$, this is a closed but noncompact Lagrangian submanifold of $T^{*} X$. To properly obtain an object of $F u k\left(T^{*} X\right)$, we must endow $L_{U, f}$ with a brane structure. This consists of a grading (or lifting of its squared phase) and relative pin structure. We check that standard Lagrangians carry canonical brane structures with respect to canonical background classes. We make $L_{U, f}$ an object of $F u k\left(T^{*} X\right)$ by equipping it with its canonical brane structure.

What is not immediately clear is what our $A_{\infty}$-functor should do with morphisms. To answer this, we first identify $\operatorname{Open}(X)$ with an $A_{\infty}$-category $\operatorname{Mor}(X)$ built out of the Morse theory of open subsets of $X$ equipped with defining functions for their complements. The construction of $\operatorname{Mor}(X)$ is a generalization of Fukaya's Morse $A_{\infty}$-category of a manifold. As with $\operatorname{Open}(X)$, the objects of $\operatorname{Mor}(X)$ are pairs $(U, m)$, where $U \subset X$ is open and $m: X \rightarrow \mathbb{R}_{\geq 0}$ is a defining function for $X \backslash U$. As usual, it is convenient to set $f=\log m$ as a function on $U$. For a finite collection of objects $\left(U_{i}, m_{i}\right)$ of $\operatorname{Mor}(X)$ indexed by $i \in \mathbb{Z} /(d+1) \mathbb{Z}$, the morphisms and composition maps among the objects encode the moduli spaces of maps from trivalent trees into $X$ that take edges to gradient lines of the functions $f_{j}-f_{i}$ with respect to some Riemannian metric on $X$. For example, the morphism complexes 
are generated by the critical points of Morse functions on certain open subsets, and the differentials are given by counting isolated gradient lines.

There are several delicate aspects to working out the details of this picture. As usual with such a construction, we must be sure that the functions $f_{i}$ and the Riemannian metric are sufficiently generic to ensure we have well-behaved moduli spaces. But in our situation, we must also be sure that the gradient vector fields of the differences $f_{i+1}-f_{i}$ are not wild at the boundaries of their domains $U_{i} \cap U_{i+1}$. To accomplish this, we employ techniques of stratification theory to move the boundaries and functions into a sufficiently transverse arrangement. Then there will be an open, convex space of Riemannian metrics such that the resulting moduli spaces are well-behaved. The upshot is that we obtain an $A_{\infty}$-structure on $\operatorname{Mor}(X)$ whose composition maps count so-called gradient trees for Morse functions on certain open subsets of $X$. Furthermore, an application of arguments of Kontsevich-Soibelman [25] from homological perturbation theory provides a quasiequivalence

$$
\operatorname{Open}(X) \simeq \operatorname{Mor}(X)
$$

Finally, we embed the Morse $A_{\infty}$-category $\operatorname{Mor}(X)$ into the Fukaya $A_{\infty}$-category $F u k\left(T^{*} X\right)$ as follows. Let $\left(U_{i}, m_{i}\right)$ be a collection of objects of $\operatorname{Mor}(X)$ indexed by $i \in \mathbb{Z} /(d+1) \mathbb{Z}$, and let $L_{U_{i}, f_{i}}$ be the corresponding collection of standard branes of $F u k\left(T^{*} X\right)$, where as usual $f_{i}=\log m_{i}$. After carefully perturbing the objects, we check that the moduli spaces of gradient trees for the former collection may be identified with the moduli spaces of pseudoholomorphic polygons for the latter. When all of the open sets $U_{i}$ are the entire manifold $X$, this is a theorem of FukayaOh [9]. These authors have identified the Morse $A_{\infty}$-category of the manifold $X$ and the Fukaya $A_{\infty}$-category of graphs in $T^{*} X$. To generalize this to arbitrary open sets, we employ the following strategy. First, using area bounds, we check that all pseudoholomophic maps with boundary on our standard branes in fact have boundary in a prescribed region. Next, we dilate our standard branes so that the theorem of Fukaya-Oh identifies the relevant moduli subspaces. Finally, we check that the homogeneity of the area bounds guarantees that no critical event occurs during the dilation. Thus we obtain an $A_{\infty}$-embedding

$$
\operatorname{Mor}(X) \hookrightarrow F u k\left(T^{*} X\right) .
$$

Putting together the above functors gives a quasi-embedding of the $A_{\infty}$-category $\operatorname{Sh}(X)$ of constructible complexes of sheaves on $X$ into the $A_{\infty}$-category of twisted complexes $T w F u k\left(T^{*} X\right)$ in the Fukaya category of $T^{*} X$. Taking the underlying cohomology categories gives an embedding of the corresponding derived categories.

For future applications, it is useful to know where the embedding takes other objects and morphisms. In particular, we would like to know not only where it takes standard sheaves on open submanifolds, but also standard sheaves on arbitrary submanifolds. One approach to this problem is to express standard sheaves on arbitrary submanifolds in terms of standard sheaves on open submanifolds, and then to check what the relevant distinguished triangles of constructible sheaves look like under the embedding. This requires identifying certain cones in the Fukaya category with symplectic surgeries. Rather than taking this route, we will instead show in the final section that we may explicitly extend the domain of the embedding to include standard sheaves on arbitrary submanifolds and morphisms between them. This has the added value that given constructible sheaves on a stratification, it obviates 
the need to further refine the stratification in order to construct the embedding: one may use the standard sheaves themselves as a generating set.

Consider the standard sheaf $R i_{*} \mathcal{L}_{Y}$ associated to a local system $\mathcal{L}_{Y}$ on an arbitrary submanifold $i: Y \hookrightarrow X$. Suppose that we are given a defining function $m: X \rightarrow \mathbb{R}_{\geq 0}$ for the boundary $\partial Y \subset X$. Recall that we define the standard Lagrangian $L_{Y, m} \subset T^{*} X$ to be the fiberwise sum

$$
L_{Y, f}=T_{Y}^{*} X+\Gamma_{d f},
$$

where $T^{*} X_{Y} \subset T^{*} X$ is the conormal bundle to $Y$ and $\Gamma_{d f} \subset T^{*} X$ is the graph of the differential of $f=\log m$. We write $L_{Y, f, \mathcal{L}_{Y}}$ for the corresponding standard object of $F u k\left(T^{*} X\right)$ given by $L_{Y, f}$ equipped with its canonical brane structure and the pullback of the flat vector bundle $\mathcal{L}_{Y} \otimes o r_{X} \otimes o r_{Y}^{-1}$, where $o r_{X}, o r_{Y}$ denote the orientation bundles of $X, Y$, respectively. The main consequence of the final section is the following.

Theorem 1.2.1. Under the embedding $D_{c}(X) \hookrightarrow D F u k\left(T^{*} X\right)$, the image of the standard sheaf $R i_{*} \mathcal{L}_{Y}$ is canonically isomorphic to the standard brane $L_{Y, f, \mathcal{L}_{Y}}$.

1.3. Mirror symmetry. The connection of this current work to mirror symmetry is somewhat speculative, though several appearances of constructible sheaves in the context of mirror symmetry deserve mention.

First, the announced results of Bondal and Bondal-Ruan [4] relate the derived categories of coherent sheaves on toric Fano varieties with the Fukaya-Seidel category on the Landau-Ginzburg side. Their method is to establish equivalences of both with the derived category of constructible sheaves on a torus with respect to a specific (non-Whitney) stratification determined by the superpotential. One can view the result of Bondal-Ruan from the perspective developed in this paper by identifying $\left(\mathbb{C}^{*}\right)^{n}$ with $T^{*}\left(\left(S^{1}\right)^{n}\right)$.

Second, Kapustin-Witten [16] place the geometric Langlands program in the context of topological quantum field theory. In particular, they relate the harmonic analysis of the geometric Langlands program to mirror symmetry by equating Hecke operators on $\mathcal{D}$-modules with 't Hooft operators acting on branes. In this setting, one may interpret the results of this paper as lending some mathematical evidence to this physical perspective. For example, according to Kapustin-Witten [16, given a generic eigen-brane for the 't Hooft operators, there is a corresponding regular, holonomic Hecke eigen- $\mathcal{D}$-module. One might hope to provide an explicit construction of the eigen- $\mathcal{D}$-module by first identifying the eigen-brane as the microlocalization of some constructible sheaf, and then applying the Riemann-Hilbert correspondence.

Third, braid group actions have been an active area of interest, especially in the context of branes in the cotangent bundle of flag varieties $\mathcal{B}$. In the case of coherent sheaves, braid group actions on $D_{\text {coh }}^{b}\left(T^{*} \mathcal{B}\right)$ have been studied by many authors (see for example Seidel-Thomas [34]). One may use the results of this paper to construct the corresponding actions in the symplectic context. Namely, under the embedding of this paper, the kernels giving the usual braid group action on the constructible derived category $D_{c}(\mathcal{B})$ (see for example Rouquier [31]) induce a corresponding action on $\operatorname{DFuk}\left(T^{*} \mathcal{B}\right)$.

Fourth, the work of Kontsevich-Soibelman [26] and Gross-Siebert [14] paints the large complex structure limit of a Calabi-Yau $n$-fold as a collapse into a real $n$-fold with integral affine structure and a Monge-Ampère metric. The complex $n$-fold is recovered from the limit manifold as a quotient of the tangent (or cotangent) bundle 
by the lattice of integer tangent vectors. It is intriguing to imagine a quotient construction creating a torus fibration from the cotangent bundle.

Finally, it would be interesting to understand whether our result is the local picture of a relationship that holds more generally for compact symplectic manifolds. One may consider modules over the deformation quantization as a global analogue of constructible sheaves. (See for example Kontsevich [24], Kashiwara 18, or Polesello-Schapira 30.) Clear comparisons can then be made between such modules and the Fukaya category. There is great interest in understanding more precisely how to interpolate between the local nature of the modules and the global nature of the Fukaya category.

\section{2. $A_{\infty}$-CATEGORIES}

Here we collect standard material concerning $A_{\infty}$-categories, dg categories, and triangulated categories. Our reference is Chapter 1 of Seidel's book [33].

2.1. Preliminaries. Our aim here is not to recall complete definitions, but only to establish notation.

Let $\mathcal{A}$ be a (not necessarily unital) $A_{\infty}$-category with a set of objects $O b \mathcal{A}$, a $\mathbb{Z}$-graded vector space of morphisms $\operatorname{hom}_{\mathcal{A}}\left(X_{0}, X_{1}\right)$, and composition maps

$$
\mu_{\mathcal{A}}^{d}: \operatorname{hom}_{\mathcal{A}}\left(X_{0}, X_{1}\right) \otimes \cdots \otimes \operatorname{hom}_{\mathcal{A}}\left(X_{d-1}, X_{d}\right) \rightarrow \operatorname{hom}_{\mathcal{A}}\left(X_{0}, X_{d}\right)[2-d] \text { for } d \geq 1 \text {. }
$$

A dg category is an $A_{\infty}$-category $\mathcal{A}$ whose higher composition maps $\mu_{\mathcal{A}}^{d}$ for $d \geq 3$ are equal to zero.

Let $H(\mathcal{A})$ denote the $\mathbb{Z}$-graded cohomological category of $\mathcal{A}$ with a set of objects $O b H(\mathcal{A})=O b \mathcal{A}$ and a $\mathbb{Z}$-graded vector space of morphisms

$$
\operatorname{hom}_{H(\mathcal{A})}\left(X_{0}, X_{1}\right)=H\left(\operatorname{hom}_{\mathcal{A}}\left(X_{0}, X_{1}\right), \mu_{\mathcal{A}}^{1}\right) .
$$

Let $H^{0}(\mathcal{A})$ denote the ungraded cohomological category with a set of objects $O b H^{0}(\mathcal{A})=O b \mathcal{A}$ and a vector space of morphisms

$$
\operatorname{hom}_{H^{0}(\mathcal{A})}\left(X_{0}, X_{1}\right)=H^{0}\left(\operatorname{hom}_{\mathcal{A}}\left(X_{0}, X_{1}\right), \mu_{\mathcal{A}}^{1}\right) .
$$

An $A_{\infty}$-category is said to be cohomologically unital or c-unital if $H(\mathcal{A})$ is unital.

Let $\mathcal{F}: \mathcal{A} \rightarrow \mathcal{B}$ be an $A_{\infty}$-functor between $A_{\infty}$-categories with map on objects $\mathcal{F}: O b \mathcal{A} \rightarrow O b \mathcal{B}$, and morphism maps

$\mathcal{F}^{d}: \operatorname{hom}_{\mathcal{A}}\left(X_{0}, X_{1}\right) \otimes \cdots \otimes \operatorname{hom}_{\mathcal{A}}\left(X_{d-1}, X_{d}\right) \rightarrow \operatorname{hom}_{\mathcal{B}}\left(\mathcal{F} X_{0}, \mathcal{F} X_{d}\right)[1-d]$, for $d \geq 1$.

An $A_{\infty}$-functor is said to be $c$-unital if $H(\mathcal{F})$ is unital.

Throughout what follows, we assume that all $A_{\infty}$-categories are c-unital, and all $A_{\infty}$-functors are c-unital. We say that an $A_{\infty}$-functor $\mathcal{F}$ is a quasi-equivalence if the induced functor $H(\mathcal{F})$ is an equivalence. We say that $\mathcal{F}$ is a quasi-embedding if $H(\mathcal{F})$ is full and faithful.

2.2. $A_{\infty}$-modules. Let $C h$ denote the dg category of chain complexes, considered as an $A_{\infty}$-category.

Given an $A_{\infty}$-category $\mathcal{A}$, an $A_{\infty}$-module over $\mathcal{A}$ is an $A_{\infty}$-functor $\mathcal{A}^{\text {opp }} \rightarrow C h$. Let $\bmod (\mathcal{A})$ denote the $A_{\infty}$-category of $A_{\infty}$-modules over $\mathcal{A}$.

The functor category $\bmod (\mathcal{A})$ inherits much of the structure of the target category $C h$. For example, $\bmod (\mathcal{A})$ is a $\operatorname{dg}$ category, and its cohomological category $H^{0}(\bmod (\mathcal{A}))$ is a triangulated category. In particular, we have the obvious shift functor $S$ on modules and the cohomological notion of exact triangle of modules. 
Note that the shift functor may be recovered by taking the cone of the zero morphism to the trivial module, or to the cone of the identity morphism of any module.

For any object $Y \in O b \mathcal{A}$, we have the $A_{\infty}$-module $\mathcal{Y}(X)=h_{\mathcal{A}}(X, Y)$ with $\mu_{\mathcal{Y}}^{d}=\mu_{\mathcal{A}}^{d}$. This provides an $A_{\infty}$-Yoneda embedding $\mathcal{J}: \mathcal{A} \rightarrow \bmod (\mathcal{A})$ which is cohomologically full and faithful. Since the ambient category $\bmod (\mathcal{A})$ is a $\operatorname{dg}$ category, the image $\mathcal{J}(\mathcal{A})$ of the Yoneda embedding is as well. Thus each $A_{\infty}$ category $\mathcal{A}$ is canonically quasi-equivalent to a dg category $\mathcal{J}(\mathcal{A})$.

2.3. Triangulated $A_{\infty}$-categories. Given an $A_{\infty}$-category $\mathcal{A}$, an exact triangle in $H(\mathcal{A})$ is defined to be any diagram in $H(A)$ which becomes isomorphic to an exact triangle of $H(\bmod (\mathcal{A}))$ under the Yoneda embedding. A shift $S X$ of an object $X$ is any object which becomes isomorphic to the shift in $H(\bmod (\mathcal{A}))$ under the Yoneda embedding.

A nonempty $A_{\infty}$-category $\mathcal{A}$ is said to be triangulated if the following hold:

(1) Every morphism in $H^{0}(\mathcal{A})$ can be completed to an exact triangle in $H(\mathcal{A})$. In particular, every object $X$ has a shift $S X$.

(2) For each object $X$, there is an object $\tilde{X}$ such that $S \tilde{X} \simeq X$ in $H^{0}(\mathcal{A})$.

If $\mathcal{A}$ is a triangulated $A_{\infty}$-category, then $H^{0}(\mathcal{A})$ is a triangulated category in the usual sense. Furthermore, if $\mathcal{F}: \mathcal{A} \rightarrow \mathcal{B}$ is an $A_{\infty}$-functor between triangulated $A_{\infty}$-categories, then $H^{0}(\mathcal{F})$ is an exact functor.

Let $\mathcal{A}$ be a full $A_{\infty}$-subcategory of a triangulated $A_{\infty}$-category $\mathcal{B}$. The triangulated $A_{\infty}$-subcategory of $\mathcal{B}$ generated by $\mathcal{A}$ is the smallest full subcategory $\overline{\mathcal{A}}$ that contains $\mathcal{A}$, is closed under cohomological isomorphism, and is itself triangulated.

A triangulated envelope of a nonempty $A_{\infty}$-category $\mathcal{A}$ is a pair $(\overline{\mathcal{A}}, \mathcal{F})$ consisting of a triangulated $A_{\infty}$-category $\overline{\mathcal{A}}$ and a cohomologically full and faithful functor $\mathcal{F}: \mathcal{A} \rightarrow \overline{\mathcal{A}}$ such that the objects in the image of $\mathcal{F}$ generate $\overline{\mathcal{A}}$. The triangulated category $H^{0}(\overline{\mathcal{A}})$ is independent of the choice of envelope up to exact equivalence. It is sometimes called the derived category of $\mathcal{A}$ and is denoted by $D(\mathcal{A})$, but we will sometimes reserve this to mean a localized version of $H^{0}(\overline{\mathcal{A}})$. Thus when we use the term derived category and the notation $D(\mathcal{A})$, we will be explicit about what is intended.

2.4. Twisted complexes. There are two standard constructions of a triangulated envelope: (i) the full subcategory of $\bmod (\mathcal{A})$ generated by the image of the Yoneda embedding, and (ii) the $A_{\infty}$-category of twisted complexes $T w \mathcal{A}$.

In this paper, we adopt the approach of twisted complexes. The explicit construction of $T w \mathcal{A}$ will play no role, only the following formal properties.

First, $T w \mathcal{A}$ is a triangulated $A_{\infty}$-category. There is a canonical $A_{\infty}$-functor $\iota: \mathcal{A} \rightarrow T w \mathcal{A}$ such that $\iota$ is injective on objects, on morphisms we have

$$
\operatorname{hom}_{\mathcal{A}}\left(X_{0}, X_{1}\right)=\operatorname{hom}_{T w \mathcal{A}}\left(\iota X_{0}, \iota X_{1}\right),
$$

and the composition maps $\mu_{\mathcal{A}}^{d}$ and $\mu_{T w \mathcal{A}}^{d}$ coincide for objects of $\mathcal{A}$ and their images under $\iota$. In short, we may identify $\mathcal{A}$ with its image under $\iota$.

We also have the following:

(1) If $\mathcal{A}$ is c-unital, then $T w \mathcal{A}$ and $\iota$ are as well.

(2) $T w \mathcal{A}$ is generated by $\mathcal{A}$.

Furthermore, any $A_{\infty}$-functor $\mathcal{F}: \mathcal{A} \rightarrow \mathcal{B}$ extends to an $A_{\infty}$-functor $T w \mathcal{F}$ : $T w \mathcal{A} \rightarrow T w \mathcal{B}$ satisfying the following:

(1) If $\mathcal{F}$ is c-unital, then $T w \mathcal{F}$ is as well. 
(2) If $\mathcal{F}$ is cohomologically full and faithful, then $T w \mathcal{F}$ is as well.

(3) If $\mathcal{F}$ is a quasi-equivalence, then $T w \mathcal{F}$ is as well.

2.5. Homological perturbation theory. Here we recall the general picture of homological perturbation theory as summarized by Seidel 33.

Let $\mathcal{B}$ be an $A_{\infty}$-category. Suppose that for each pair of objects $\left(X_{0}, X_{1}\right)$, we have a chain complex $\left(h_{\mathcal{A}}\left(X_{0}, X_{1}\right), \mu_{\mathcal{A}}^{1}\right)$, chain maps

$$
\mathcal{F}^{1}: \operatorname{hom}_{\mathcal{A}}\left(X_{0}, X_{1}\right) \rightarrow \operatorname{hom}_{\mathcal{B}}\left(X_{0}, X_{1}\right), \quad \mathcal{G}^{1}: \operatorname{hom}_{\mathcal{B}}\left(X_{0}, X_{1}\right) \rightarrow \operatorname{hom}_{\mathcal{A}}\left(X_{0}, X_{1}\right)
$$

of degree 0 , and a linear map

$$
T^{1}: \operatorname{hom}_{\mathcal{B}}\left(X_{0}, X_{1}\right) \rightarrow \operatorname{hom}_{\mathcal{B}}\left(X_{0}, X_{1}\right)
$$

of degree -1 such that

$$
\mathcal{F}^{1} \circ \mathcal{G}^{1}-i d=\mu_{B}^{1} T^{1}+T^{1} \mu_{\mathcal{B}}^{1} .
$$

In the preceding set-up, the subscript $\mathcal{A}$ is simply suggestive notation. The main statement of homological perturbation theory is that there is an explicit construction of an $A_{\infty}$-category $\mathcal{A}$ with objects $O b \mathcal{A}=O b \mathcal{B}$, and morphism complexes the given $\left(\operatorname{hom}_{\mathcal{A}}\left(X_{0}, X_{1}\right), \mu_{\mathcal{A}}^{1}\right)$. Furthermore, there are $A_{\infty}$-functors $\mathcal{F}: \mathcal{A} \rightarrow \mathcal{B}$, $\mathcal{G}: \mathcal{B} \rightarrow \mathcal{A}$ which are the identity on objects and have first-order terms the given $\mathcal{F}^{1}, \mathcal{G}^{1}$. Finally, there is a homotopy between $\mathcal{F} \circ \mathcal{G}$ and $i d_{\mathcal{B}}$ which starts with the given $T^{1}$.

We will use the special case of this construction when $\mathcal{G}^{1}$ is an idempotent $\pi^{1}$ and $\mathcal{F}^{1}$ is the inclusion $i^{1}$ of the image of $\pi^{1}$. In other words, for each pair of objects $\left(X_{0}, X_{1}\right)$, we have a chain map

$$
\pi^{1}: \operatorname{hom}_{\mathcal{B}}\left(X_{0}, X_{1}\right) \rightarrow \operatorname{hom}_{\mathcal{B}}\left(X_{0}, X_{1}\right)
$$

of degree 0 such that $\pi^{1} \circ \pi^{1}=\pi^{1}$, and a linear map

$$
T^{1}: \operatorname{hom}_{\mathcal{B}}\left(X_{0}, X_{1}\right) \rightarrow \operatorname{hom}_{\mathcal{B}}\left(X_{0}, X_{1}\right)
$$

of degree -1 such that

$$
i^{1} \circ \pi^{1}-i d=\mu_{B}^{1} T^{1}+T^{1} \mu_{\mathcal{B}}^{1} .
$$

In this case, if we take

$$
\operatorname{hom}_{\mathcal{A}}\left(X_{0}, X_{1}\right)=\pi^{1}\left(\operatorname{hom}_{\mathcal{B}}\left(X_{0}, X_{1}\right)\right),
$$

then the resulting $A_{\infty}$-functors $i: \mathcal{A} \rightarrow \mathcal{B}, \pi: \mathcal{B} \rightarrow \mathcal{A}$ are quasi-equivalences.

\section{AnALytic-GeOMETRIC CATEgORIES}

When working with sheaves on a manifold $X$, it is often useful if not indispensable to restrict to subsets of $X$ that have strong finiteness properties. In this section, we collect basic material from the theory of subanalytic sets that plays a role in what follows. All of the results and arguments that we use hold in the context of analytic-geometric categories. Since this seems to be a natural level of generality, we adopt it as our framework. What follows is a brief summary of relevant results from van den Dries-Miller [36. For a discussion of subanalytic sets alone, see BierstoneMilman [3]. The reader may prefer to ignore the generality of analytic-geometric categories and consider all discussions to take place in the subanalytic category.

Throughout what follows, all manifolds are assumed to be real analytic unless otherwise specified. 
3.1. Basic definitions. To give an analytic-geometric category $\mathcal{C}$ is to equip each manifold $M$ with a collection $\mathcal{C}(M)$ of subsets of $M$ satisfying the following properties:

(1) $\mathcal{C}(M)$ is a Boolean algebra of subsets with $M \in \mathcal{C}(M)$.

(2) If $A \in \mathcal{C}(M)$, then $A \times \mathbb{R} \in \mathcal{C}(M \times \mathbb{R})$.

(3) If $f: M \rightarrow N$ is a proper analytic map and $A \in \mathcal{C}(M)$, then $f(A) \in \mathcal{C}(N)$.

(4) If $A \subset M$, and $\left(U_{i}\right)_{i \in I}$ is an open covering of $M$, then $A \in \mathcal{C}(M)$ if and only if $A \cap U_{i} \in \mathcal{C}\left(U_{i}\right)$, for all $i \in I$.

(5) For every bounded set $A \in \mathcal{C}(\mathbb{R})$, the boundary $\partial A$ is finite.

Given the above data, one defines a category $\mathcal{C}$ as follows. An object is a pair $(A, M)$ with $M$ a manifold and $A \in \mathcal{C}(M)$. A morphism $(A, M) \rightarrow(B, N)$ is a continuous map $f: A \rightarrow B$ whose graph $\Gamma(F) \subset A \times B$ belongs to $\mathcal{C}(M \times$ $N)$. Objects of $\mathcal{C}$ are called $\mathcal{C}$-sets, and morphisms are called $\mathcal{C}$-maps. When the codomain of a map is $\mathbb{R}$, we refer to it as a function.

The basic example of an analytic-geometric category is the subanalytic category $\mathcal{C}_{a n}$ of subanalytic sets and continuous maps with subanalytic graphs. For any analytic-geometric category $\mathcal{C}$, the subanalytic subsets of any manifold $M$ belong to $\mathcal{C}(M)$.

3.2. Background results. Most of the fundamental results about subanalytic sets hold in any analytic-geometric category (although it is unknown whether the uniformization and rectilinearization properties of subanalytic sets have analogues). We limit our discussion here to include only those results which we use.

3.2.1. Derivatives. Given a manifold $M$, the tangent bundle $T M$ and cotangent bundle $T^{*} M$ are also manifolds. Given a $C^{1}$ submanifold $A \subset M$, let $T A \subset T M$ denote its tangent bundle and $T_{A}^{*} M \subset T^{*} M$ its conormal bundle.

Lemma 3.2.1. If $A \in \mathcal{C}(M)$ is a $C^{1}$ submanifold of $M$, then $T A \in \mathcal{C}(T M)$ and $T_{A}^{*} M \in \mathcal{C}\left(T^{*} M\right)$. If $f: M \rightarrow N$ is a $\mathcal{C}$-map of class $C^{1}$, its differential $T f: T M \rightarrow$ $T N$ is a $\mathcal{C}$-map.

3.2.2. Whitney stratifications. Let $X, Y$ be $C^{1}$ submanifolds of a manifold $M$, and let $x \in X$. The triple $(Y, X, x)$ is said to satisfy Whitney's condition if given any sequences of points $x_{i} \in X$ and $y_{i} \in Y$ each converging to $x$, such that in some local coordinate chart the secant lines $\ell_{i}=\overline{x_{i} y_{i}}$ converge to some line $\ell$ and the tangent planes $T_{y_{i}} Y$ converge to some plane $\tau$, we have $\ell \subset \tau$. The pair $(Y, X)$ is said to satisfy Whitney's condition if for all $x \in X$, the triples $(Y, X, x)$ satisfy the condition.

A $C^{p}$ stratification of a manifold $M$ consists of a locally finite collection $\mathcal{S}=\left\{S_{\alpha}\right\}$ of locally closed $C^{p}$ submanifolds $S_{\alpha} \subset M$ called strata satisfying

(1) (covering) $X=\bigcup_{\alpha} S_{\alpha}$,

(2) (pairwise disjoint) $S_{\alpha} \cap S_{\beta}=\emptyset$, for $\alpha \neq \beta$,

(3) (axiom of frontier) $\overline{S_{\alpha}} \cap S_{\beta} \neq \emptyset$ if and only if $S_{\beta} \subset \overline{S_{\alpha}}$.

A $C^{p}$ stratification $\mathcal{S}=\left\{S_{\alpha}\right\}$ of $M$ is called a $C^{p}$ Whitney stratification if $\left(S_{\alpha}, S_{\beta}\right)$ satisfies Whitney's condition for all $\alpha, \beta$.

A stratification $\mathcal{S}$ of $M$ is said to be compatible with a collection $\mathcal{A}$ of subsets of $M$ if $S \cap A \neq \emptyset$ implies $S \subset A$, for all $S \in \mathcal{S}, A \in \mathcal{A}$. 
Given a map $f: M \rightarrow N$ between manifolds, a $C^{p}$ Whitney stratification of $f$ is a pair $(\mathcal{S}, \mathcal{T})$ where $\mathcal{S}$ and $\mathcal{T}$ are $C^{p}$ Whitney stratifications of $M$ and $N$ respectively such that for each $S \in \mathcal{S}$, the map $\left.f\right|_{S}: S \rightarrow N$ is a $C^{p}$ submersion with $f(S) \in \mathcal{T}$.

Proposition 3.2.2. Let $X \in \mathcal{C}(M)$ be closed, and $p$ a positive integer.

(1) For every locally finite collection $\mathcal{A} \subset \mathcal{C}(M)$, there is a $C^{p}$ Whitney stratification $\mathcal{S} \subset \mathcal{C}(M)$ of $M$ which is compatible with $\mathcal{A}$ and has connected strata.

(2) Let $f: M \rightarrow N$ be a proper $\mathcal{C}$-map, and let $\mathcal{A} \subset \mathcal{C}(M), \mathcal{B} \subset \mathcal{C}(N)$ be locally finite collections. Then there is a $C^{p}$ Whitney stratification $(\mathcal{S}, \mathcal{T})$ of $f$ with connected strata such that $\mathcal{S} \subset \mathcal{C}(M)$ is compatible with $\mathcal{A}$ and $\mathcal{T} \subset \mathcal{C}(N)$ is compatible with $\mathcal{B}$.

Remark 3.2.3. In Proposition $3.2 .2(1)$, we may find a stratification $\mathcal{S} \subset \mathcal{C}(M)$ of $M$ such that each stratum $S \in \mathcal{S}$ is a $\left(C^{p}, \mathcal{C}\right)$ cell in $M$. In particular, each $S \in \mathcal{S}$ will be $C^{p}$ diffeomorphic to $\mathbb{R}^{d}$, where $d=\operatorname{dim} S$.

To a Whitney stratification $\mathcal{S}=\left\{S_{\alpha}\right\}$ of $M$, we associate the conical set $\Lambda_{\mathcal{S}} \subset$ $T^{*} M$ given by the union

$$
\Lambda_{\mathcal{S}}=\bigcup_{\alpha} T_{S_{\alpha}}^{*} M
$$

By Lemma 3.2.1 if $\mathcal{S} \subset \mathcal{C}(M)$, then $\Lambda_{\mathcal{S}} \in \mathcal{C}\left(T^{*} X\right)$. If a stratification $\mathcal{S}_{1}$ of $M$ is compatible with another $\mathcal{S}_{2}$, then $\Lambda_{\mathcal{S}_{2}} \subset \Lambda_{\mathcal{S}_{1}}$.

For a function $f: X \rightarrow \mathbb{R}$, we say that $x \in X$ is a $\Lambda_{\mathcal{S}}$-critical point of $f$ if we have $d f(x) \in \Lambda_{\mathcal{S}}$. We say that $r \in \mathbb{R}$ is a $\Lambda_{\mathcal{S}}$-critical value of $f$ if there is a $\Lambda_{\mathcal{S}}$-critical point $x \in X$ such that $r=f(x)$; otherwise we say that $r$ is a $\Lambda_{\mathcal{S}}$-regular value.

Lemma 3.2.4. If $f$ is a $\mathcal{C}$-map, then the $\Lambda_{\mathcal{S}}$-critical values of $f$ form a discrete subset of $\mathbb{R}$.

Proof. The $\Lambda_{\mathcal{S}}$-regular values are a dense open $\mathcal{C}$-subset of $\mathbb{R}$.

\subsubsection{Curve selection lemma.}

Proposition 3.2.5. If $A \in \mathcal{C}(M)$, and $x \in \bar{A} \backslash A$, then there is a $\mathcal{C}$-map $\gamma:[0,1) \rightarrow$ $M$ such that $\gamma(0,1) \subset A$ and $\gamma(0)=x$. Furthermore, if $p$ is a positive integer, $\gamma$ can be chosen to be injective and of class $C^{p}$.

3.2.4. Defining functions. For a function $m: M \rightarrow \mathbb{R}$, let $Z(m)=\{x \in M \mid m(x)=$ $0\}$. Given a subset $A \subset M$, we call any function $m: M \rightarrow \mathbb{R}_{\geq 0}$ with $Z(m)=A$ a defining function for $A$. The space of defining functions for $A$ is convex.

Proposition 3.2.6. If $A \in \mathcal{C}(M)$ is closed and $p$ is a positive integer, then there is a $\mathcal{C}$-map $m: M \rightarrow \mathbb{R}$ of class $C^{p}$ with $A=Z(m)$.

Using defining functions, one can construct bump functions as follows. For $A_{1}, A_{2} \in \mathcal{C}(M)$ disjoint and closed with defining functions $m_{1}, m_{2}$ respectively, define $g_{i}=m_{i}^{2} /\left(1+m_{i}^{2}\right)$ for $i=1,2$. Then the function $b=\left(g_{1}+g_{1} g_{2}\right) /\left(g_{1}+g_{2}\right)$ satisfies $Z(b)=A_{1}, Z(b-1)=A_{2}$, and $0 \leq b \leq 1$. The existence of bump functions implies the existence of partitions of unity and constructions which devolve from them. 
Remark 3.2.7. In simple situations, one can explicitly produce $C^{p}$ bump functions with compact support such as

$$
b(x)=\left\{\begin{array}{c}
\left(1-x^{2}\right)^{p+1}, \quad x^{2}<1, \\
0, \quad \text { otherwise }
\end{array}\right.
$$

\section{Constructible sheaves}

Let $X$ be a real analytic manifold. All subsets of $X$ are assumed to belong to some fixed analytic-geometric category unless otherwise specified.

Let $\mathbb{C}_{X}$ be the sheaf of locally-constant complex-valued functions on $X$. By a sheaf on $X$, we will always mean a sheaf of $\mathbb{C}_{X}$-modules. A sheaf $\mathcal{F}$ is said to be constructible if there exists a Whitney stratification of $X$ such that the restriction of $\mathcal{F}$ to each stratum is locally-constant and finitely-generated.

We define the localized triangulated dg category $S h(X)$ of complexes of sheaves with bounded constructible cohomology as follows. First, we have the naive triangulated dg category $S h_{\text {naive }}(X)$ whose objects are complexes of sheaves with bounded constructible cohomology and whose morphisms are the usual complexes of morphisms. Then, we take $S h(X)$ to be the dg quotient of $S h_{\text {naive }}(X)$ with respect to the subcategory $\mathcal{N}$ of acyclic objects [23]. As explained in [5], this can be achieved by simply adding a homotopy between zero and the identity to the endomorphism complex of each object of $\mathcal{N}$. The ungraded cohomological category $H^{0}(S h(X))$ is the usual bounded constructible derived category $D_{c}(X)$.

We have the six standard derived functors of Grothendieck, $f_{*}, f^{*}, f_{!}, f^{!}, \otimes$ and $\mathcal{H}$ om. We similarly have the Verdier duality functor $\mathcal{D}$. Note that we only consider derived functors, though the notation does not make this explicit. We also refer to objects of $S h(X)$ as sheaves, though they are proper complexes of sheaves.

4.1. Standard objects. The most accessible objects of $S h(X)$ are the so-called standard and costandard sheaves of submanifolds. To be precise, let $i: Y \hookrightarrow X$ be the inclusion of a submanifold (with its subspace topology) with closure $\bar{Y} \subset X$ and boundary $\partial Y=\bar{Y} \backslash Y \subset X$. Note that the boundary could be a singular subset. For a local system $\mathcal{L}_{Y}$ on $Y$, we call the sheaf $i_{*} \mathcal{L}_{Y}$ a standard object, and the sheaf $i_{!} \mathcal{L}_{Y}$ a costandard object. The terminology reflects the fact that Verdier duality intertwines the two extensions

$$
\mathcal{D}_{X}\left(i_{!} \mathcal{L}_{Y}\right) \simeq i_{*} \mathcal{D}_{Y}\left(\mathcal{L}_{Y}\right) .
$$

Suppose $U \subset X$ is an open set. The complex of sections of $i_{*} \mathcal{L}_{Y}$ over $U$ is quasiisomorphic to the complex of $\mathcal{L}_{Y \text {-valued singular cochains }}$

$$
\Gamma\left(U, i_{*} \mathcal{L}_{Y}\right) \simeq C^{*}\left(U \cap Y, \mathcal{L}_{Y}\right) .
$$

Similarly, the complex of sections of $i_{!} \mathbb{C}_{Y}$ over $U$ is quasi-isomorphic to the complex of $\mathcal{L}_{Y}$-valued relative singular cochains

$$
\Gamma\left(U, i_{!} \mathbb{C}_{Y}\right) \simeq C^{*}\left(U \cap \bar{Y}, U \cap \partial Y, \mathcal{L}_{Y}\right) .
$$

4.2. Standard triangles. Fix a subset $Z \subset X$. Let $i: U \hookrightarrow Z$ be the inclusion of an open set, and $j: Y=Z \backslash U \hookrightarrow Z$ the inclusion of its complement. Note that since $U$ is open and $Y$ is closed in $Z$, we have identities $i^{!} \simeq i^{*}$ and $j_{!} \simeq j_{*}$. For any sheaf $\mathcal{F}$ on $Z$, we have the standard distinguished triangles

$$
j_{*} j^{!} \mathcal{F} \rightarrow \mathcal{F} \rightarrow i_{*} i^{*} \mathcal{F} \stackrel{[1]}{\rightarrow}, \quad i_{*} i^{\prime} \mathcal{F} \rightarrow \mathcal{F} \rightarrow j_{*} j^{*} \mathcal{F} \stackrel{[1]}{\rightarrow} .
$$


For example, if we take $\mathcal{F}$ to be $\mathbb{C}_{Z}$ and take the cohomology of global sections, we obtain from these two the standard long exact sequences

$$
H^{*}(Z, U) \rightarrow H^{*}(Z) \rightarrow H^{*}(U) \stackrel{[1]}{\rightarrow}, \quad H^{*}(Z, Y) \rightarrow H^{*}(Z) \rightarrow H^{*}(Y) \stackrel{[1]}{\rightarrow} .
$$

We also have distinguished triangles associated to truncation functors. Let $\tau_{\leq \ell}$ be the functor which assigns to a complex $\mathcal{F}$ the truncated complex

$$
\cdots \rightarrow \mathcal{F}^{\ell-1} \rightarrow \operatorname{ker}\left(d_{\ell}\right) \rightarrow 0 \rightarrow \cdots .
$$

The natural map $\tau_{\leq \ell} \mathcal{F} \rightarrow \mathcal{F}$ induces an isomorphism on cohomology sheaves in degrees less than or equal to $\ell$. Let $\tau_{>\ell}$ be the functor which assigns to a complex $\mathcal{F}$ the truncated complex

$$
\cdots \rightarrow 0 \rightarrow \operatorname{im}\left(d_{\ell}\right) \rightarrow \mathcal{F}^{\ell+1} \rightarrow \cdots .
$$

The natural map $\mathcal{F} \rightarrow \tau_{>\ell} \mathcal{F}$ induces an isomorphism on cohomology sheaves in degrees greater than $\ell$. We have a distinguished triangle

$$
\tau_{\leq \ell} \mathcal{F} \rightarrow \mathcal{F} \rightarrow \tau_{>\ell} \mathcal{F} \stackrel{[1]}{\rightarrow} .
$$

\subsection{Standard objects generate.}

Proposition 4.3.1. Any object of $S h(X)$ is isomorphic to one obtained from shifts of standard objects by iteratively forming cones. The same is true for costandard objects.

Proof. Let $\mathcal{F}$ be an object of $S h(X)$. Fix a stratification $\mathcal{S}$ of $X$ such that the cohomology sheaves of $\mathcal{F}$ are constructible with respect to $\mathcal{S}$.

We prove the first assertion (the second is similar, or follows by Verdier duality). The proof is an induction on the strata, beginning with the open strata. Let $i_{k}: \mathcal{S}_{k} \rightarrow X$ be the inclusion of the union of the strata of dimension equal to $k$, and let $j_{<k}: \mathcal{S}_{<k} \rightarrow X$ be the inclusion of the union of the strata of dimension less than $k$.

Suppose $X$ has dimension equal to $n$. Then for the sheaf $\mathcal{F}$, we have a distinguished triangle

$$
j_{<n *} j_{<n}^{!} \mathcal{F} \rightarrow \mathcal{F} \rightarrow i_{n *} i_{n}^{*} \mathcal{F} \stackrel{[1]}{\rightarrow} .
$$

Using truncation functors, we may express the sheaf $\mathcal{F}_{n}=i_{n *} i_{n}^{*} \mathcal{F}$ by iteratively forming cones of shifted standard objects associated to the strata $\mathcal{S}_{n}$. By construction, the sheaf $\mathcal{F}_{<n}=j_{<n *} j_{<n}^{!} \mathcal{F}$ is supported on $\mathcal{S}_{<n}$.

Next we have the distinguished triangle

$$
j_{<n-1 *} j_{<n-1}^{!} \mathcal{F}_{<n} \rightarrow \mathcal{F}_{<n} \rightarrow i_{n-1 *} i_{n-1}^{*} \mathcal{F}_{<n} \stackrel{[1]}{\rightarrow} .
$$

Again, using truncation functors, we may express the sheaf $\mathcal{F}_{n-1}=i_{n-1 *} i_{n-1}^{*} \mathcal{F}_{<n}$ by iteratively forming cones of shifted standard objects associated to the strata $\mathcal{S}_{n-1}$. By construction, the sheaf $\mathcal{F}_{<n-1}=j_{<n-1 *} j_{<n-1} \mathcal{F}_{<n}$ is supported on $\mathcal{S}_{<n-1}$.

And so on. In the end, we see that $\mathcal{F}$ may be expressed by iteratively forming cones of shifted standard objects.

We have the following strengthening of the proposition.

Proposition 4.3.2. Any object of $S h(X)$ is isomorphic to one obtained from shifts of constant standard objects $i_{*} \mathbb{C}_{U}$ for open submanifolds $i: U \hookrightarrow X$ by iteratively forming cones. The same is true for constant costandard objects $i_{!} \mathbb{C}_{U}$. 
Proof. Let $\mathcal{F}$ be an object of $S h(X)$. Choose a stratification $\mathcal{T}$ of $X$ such that the cohomology sheaves of $\mathcal{F}$ are constructible with respect to $\mathcal{T}$, and the strata of $\mathcal{T}$ are cells (see Remark 3.2.3). By the previous proposition, and since the strata are cells, we need to show that for a stratum $j: T \rightarrow X$, we can realize the standard object $j_{*} \mathbb{C}_{T}$.

Let $\operatorname{Star}(T)$ be the union of all the strata of $\mathcal{T}$ which contain $T$ in their closures, and let $s: \operatorname{Star}(T) \rightarrow X$ denote its inclusion. Let $\operatorname{Star}^{\prime}(T)$ be the complement of $T$ in $\operatorname{Star}(T)$, and let $s^{\prime}: \operatorname{Star}^{\prime}(T) \rightarrow X$ denote its inclusion. Both $\operatorname{Star}(T)$ and $\operatorname{Star}^{\prime}(T)$ are open submanifolds. We have the distinguished triangle

$$
j_{*} j^{\prime} \mathbb{C}_{S \operatorname{Star}(T)} \rightarrow s_{*} \mathbb{C}_{S \operatorname{Star}(T)} \rightarrow s_{*}^{\prime} \mathbb{C}_{S \operatorname{Star}(T)} \stackrel{[1]}{\rightarrow} .
$$

Since $T \hookrightarrow \operatorname{Star}(T)$ is the inclusion of an orientable submanifold, $j^{!} \mathbb{C}_{S \operatorname{Star}(T)}$ is isomorphic to a shift of $\mathbb{C}_{T}$, and the assertion is proved.

4.4. Open submanifolds. From here on, we focus on standard objects rather than costandard objects, though there is no reason to prefer one over the other. Furthermore, we work with standard objects for open submanifolds. By Proposition 4.3.2, such objects generate the entire category $S h(X)$. Thus it suffices to work with them in proving our main theorem. While this will simplify many constructions, there is a price to pay. First, we will lose concrete touch with other objects and only understand what is happening with them in an abstract sense this has implications for applications of our main result. Second, there are contexts in which the arguments of Proposition 4.3.2 are not really acceptable - often we are presented with a fixed stratification and would prefer not to subdivide it further. To remedy both of these points, we have included a discussion in Section 7 explaining how to generalize our arguments to deal with all standard objects, not only those for open submanifolds. In what follows, we also consider standard objects with trivial coefficients and leave the case of arbitrary local systems to the reader. This is a purely expositional choice, and the reader will have no trouble extending our arguments. In any case, technically speaking, Proposition 4.3 .2 also obviates the need to consider arbitrary local systems.

For an open subset $U \subset X$, let $\Omega^{k}(U)$ denote the space of differential $k$-forms on $U$, and let $(\Omega(U), d)$ denote the de Rham complex. Define the support of a $k$-form $\omega \in \Omega^{k}(U)$ to be the smallest closed subset $\operatorname{supp}(\omega) \subset X$ such that

$$
\left.\omega\right|_{U \cap(X \backslash \operatorname{supp}(\omega))}=0 .
$$

Now consider a pair $(U, V)$, where $V \subset U \subset X$ such that $U \backslash V$ is open. Let $(\Omega(U, V), d)$ denote the relative de Rham complex of differential forms on $U \backslash V$ whose support lies in $X \backslash V$. The complex $(\Omega(U, V), d)$ calculates the relative cohomology $H^{*}(U, V)$.

Recall that for a subset $A \subset X$, we call any function $m: X \rightarrow \mathbb{R}_{\geq 0}$ with zero set $Z(m)=A$ a defining function for $A$. We define a dg category $O p e n(X)$ as follows. The objects of $\operatorname{Open}(X)$ are pairs $\mathfrak{U}=(U, m)$, where $U \subset X$ is an open set and $m: X \rightarrow \mathbb{R}_{>0}$ is a defining function for the complement $X \backslash U \mathbb{1}$ The complex of morphisms from an object $\mathfrak{U}_{0}=\left(U_{0}, m_{0}\right)$ to an object $\mathfrak{U}_{1}=\left(U_{1}, m_{1}\right)$ is the relative

\footnotetext{
${ }^{1}$ In some contexts, it might be more natural to assume that $m$ is a defining function for the boundary $\partial U \subset X$. Our requirement that $m$ vanishes on all of $X \backslash U$ plays no role outside of reducing future notation.
} 
de Rham complex

$$
\operatorname{hom}_{O \operatorname{pen}(X)}\left(\mathfrak{U}_{0}, \mathfrak{U}_{1}\right)=\left(\Omega\left(\bar{U}_{0} \cap U_{1}, \partial U_{0} \cap U_{1}\right), d\right) .
$$

Note the obvious fact that the morphisms are independent of the defining functions. Given a third object $\mathfrak{U}_{2}=\left(U_{2}, m_{2}\right)$, the composition of morphisms is the wedge product of forms

$$
\Omega\left(\bar{U}_{0} \cap U_{1}, \partial U_{0} \cap U_{1}\right) \otimes \Omega\left(\bar{U}_{1} \cap U_{2}, \partial U_{1} \cap U_{2}\right) \rightarrow \Omega\left(\bar{U}_{0} \cap U_{2}, \partial U_{0} \cap U_{2}\right) .
$$

To see this is well defined, note that the support of any such wedge product lies in $\bar{U}_{1}$, and thus since

$\left(\partial U_{0} \cap U_{2}\right) \cap \bar{U}_{1}=\left(\partial U_{0} \cap U_{2} \cap U_{1}\right) \cup\left(\partial U_{0} \cap U_{2} \cap \partial U_{1}\right) \subset\left(\partial U_{0} \cap U_{1}\right) \cup\left(U_{2} \cap \partial U_{1}\right)$, the support is disjoint from $\partial U_{0} \cap U_{2}$.

For an open subset $i: U \hookrightarrow X$, recall that $i_{*} \mathbb{C}_{U}$ denotes the standard extension of the constant sheaf on $U$.

Lemma 4.4.1. For open subsets $i_{0}: U_{0} \hookrightarrow X, i_{1}: U_{1} \hookrightarrow X$, we have a canonical quasi-isomorphism

$$
\operatorname{hom}_{S h(X)}\left(i_{0 *} \mathbb{C}_{U_{0}}, i_{1 *} \mathbb{C}_{U_{1}}\right) \simeq\left(\Omega\left(\bar{U}_{0} \cap U_{1}, \partial U_{0} \cap U_{1}\right), d\right) .
$$

The composition of morphisms coincides with the wedge product of differential forms.

Proof. By standard identities, we have canonical quasi-isomorphisms

$$
\begin{aligned}
\operatorname{hom}_{S h(X)}\left(i_{0 *} \mathbb{C}_{U_{0}}, i_{1 *} \mathbb{C}_{U_{1}}\right) & \simeq \Gamma\left(X, \mathcal{H o m}\left(i_{0 *} \mathbb{C}_{U_{0}}, i_{1 *} \mathbb{C}_{U_{1}}\right)\right) \\
& \simeq \Gamma\left(X, i_{1 *} \mathcal{H o m}\left(i_{1}^{*} i_{0 *} \mathbb{C}_{U_{0}}, \mathbb{C}_{U_{1}}\right)\right) \\
& \simeq \Gamma\left(X, i_{1 *} \mathcal{H o m}\left(\omega_{U_{1}}, i_{1}^{!} i_{0 !} \omega_{U_{0}}\right)\right) \\
& \simeq \Gamma\left(X, i_{1 *} i_{1}^{*} i_{0 !} \mathbb{C}_{U_{0}}\right) .
\end{aligned}
$$

Here we have written $\omega_{U_{0}}, \omega_{U_{1}}$ for the dualizing complexes. By de Rham's theorem, we also have a canonical quasi-isomorphism

$$
\Gamma\left(X, i_{1 *} i_{1}^{*} i_{0 !} \mathbb{C}_{U_{0}}\right) \simeq\left(\Omega\left(\bar{U}_{0} \cap U_{1}, \partial U_{0} \cap U_{1}\right), d\right) .
$$

We leave it to the reader to check the last assertion.

By the preceding lemma, we may define a dg functor $P: \operatorname{Open}(X) \rightarrow \operatorname{Sh}(X)$ by sending an object $\mathfrak{U}=(U, m)$ to the standard sheaf $i_{*} \mathbb{C}_{U}$, where $i: U \hookrightarrow X$ is the inclusion. By the preceding lemma and Proposition 4.3.2, the induced dg functor on twisted complexes $T w P: T w O p e n(X) \rightarrow S h(X)$ is a quasi-equivalence.

4.5. Smooth boundaries. In what follows, we explain how to calculate morphisms in $\operatorname{Open}(X)$ using open sets with smooth transverse boundaries. To do this, we will need to make choices of perturbation data. It will be clear that the choices range over a contractible set, and that they can be made compatibly for any finite collection of objects.

Recall that the complex of morphisms from an object $\mathfrak{U}_{0}=\left(U_{0}, m_{0}\right)$ to an object $\mathfrak{U}_{1}=\left(U_{1}, m_{1}\right)$ is the relative de Rham complex

$$
\operatorname{hom}_{O p e n(X)}\left(\mathfrak{U}_{0}, \mathfrak{U}_{1}\right)=\left(\Omega\left(\bar{U}_{0} \cap U_{1}, \partial U_{0} \cap U_{1}\right), d\right) .
$$

Our reinterpretation of this will be a complex not only quasi-isomorphic to it but in fact isomorphic to it.

First, fix a Whitney stratification $\mathcal{S}_{0}$ of $X$ compatible with the boundary $\partial U_{0} \subset$ $X$, and let $\Lambda_{\mathcal{S}_{0}} \subset T^{*} X$ be the conical conormal set associated to $\mathcal{S}_{0}$. By Lemma 3.2.4, 
there is $\bar{\eta}_{1}>0$ such that there are no $\Lambda_{0}$-critical values of $m_{1}$ in the open interval $\left(0, \bar{\eta}_{1}\right)$.

Lemma 4.5.1. For $\eta_{1} \in\left(0, \bar{\eta}_{1}\right)$, there is a compatible collection of identifications

$$
\left(\bar{U}_{0} \cap X_{m_{1}>\eta_{1}}, \partial U_{0} \cap X_{m_{1}>\eta_{1}}\right) \simeq\left(\bar{U}_{0} \cap U_{1}, \partial U_{0} \cap U_{1}\right)
$$

which are the identity on $\bar{U}_{0} \cap X_{m_{1} \geq \bar{\eta}_{1}}$.

Proof. By construction, there are no $\Lambda_{\mathcal{S}_{0}}$-critical points of the map

$$
m_{1}: X_{0<m_{1}<\bar{\eta}_{1}} \rightarrow\left(0, \bar{\eta}_{1}\right) .
$$

For $\eta_{1} \in\left(0, \bar{\eta}_{1}\right)$, we may construct a compatible collection of diffeomorphisms

$$
\left(0, \bar{\eta}_{1}\right) \rightarrow\left(\eta_{1}, \bar{\eta}_{1}\right)
$$

by integrating an appropriate collection of vector fields. Thus by the Thom isotopy lemma, we may lift these diffeomorphisms to obtain identifications

$$
\bar{U}_{0} \cap X_{m_{1}>\eta_{1}} \simeq \bar{U}_{0} \cap U_{1} .
$$

Since $\mathcal{S}_{0}$ is compatible with $\partial U_{0}$, the constructed identifications respect the pairs.

Next, choose $\eta_{1} \in\left(0, \bar{\eta}_{1}\right)$, fix the Whitney stratification $\mathcal{S}_{\eta_{1}}$ of $X$ given by the hypersurface $X_{m_{1}=\eta_{1}}$ and its complement, and let $\Lambda_{\mathcal{S}_{\eta_{1}}} \subset T^{*} X$ be the conical conormal set associated to $\mathcal{S}_{\eta_{1}}$. By Lemma 3.2.4, there is $\bar{\eta}_{0}>0$ such that there are no $\Lambda_{\mathcal{S}_{\eta_{1}}}$-critical values of $m_{0}$ in the open interval $\left(0, \bar{\eta}_{0}\right)$.

Lemma 4.5.2. For $\eta_{0} \in\left(0, \bar{\eta}_{0}\right)$, there is a compatible collection of identifications

$$
\left(X_{m_{0}>\eta_{0}} \cap X_{m_{1} \geq \eta_{1}}, X_{m_{0}>\eta_{0}} \cap X_{m_{1}=\eta_{1}}\right) \simeq\left(X_{m_{0}>0} \cap X_{m_{1} \geq \eta_{1}}, X_{m_{0}>0} \cap X_{m_{1}=\eta_{1}}\right)
$$

which are the identity on $X_{m_{0} \geq \bar{\eta}_{0}} \cap X_{m_{1} \geq \eta_{1}}$.

Proof. The argument is similar to that of the previous lemma. By construction, there are no $\Lambda_{\mathcal{S}_{\eta_{1}}}$-critical points of the map

$$
m_{0}: X_{0<m_{0}<\bar{\eta}_{0}} \rightarrow\left(0, \bar{\eta}_{0}\right) .
$$

For $\eta_{0} \in\left(0, \bar{\eta}_{0}\right)$, we may construct a compatible collection of diffeomorphisms

$$
\left(0, \bar{\eta}_{0}\right) \rightarrow\left(\eta_{1}, \bar{\eta}_{0}\right)
$$

by integrating an appropriate collection of vector fields. Thus by the Thom isotopy lemma, we may lift these diffeomorphisms to obtain identifications

$$
X_{m_{0}>0} \cap X_{m_{1} \geq \eta_{1}} \simeq X_{m_{0}>\eta_{0}} \cap X_{m_{1} \geq \eta_{1}} .
$$

Since $\mathcal{S}_{\eta_{1}}$ is compatible with $X_{m_{1}=\eta_{1}}$, the constructed identifications respect the pairs.

Putting together the two previous lemmas, we obtain a compatible collection of identifications

$$
U_{0} \cap U_{1} \simeq X_{m_{0}>\eta_{0}} \cap X_{m_{1}>\eta_{1}} .
$$

Now we have the crucial observation: for every open set $N_{0} \subset X$ containing $\partial U_{0} \cap$ $X_{m_{1} \geq \eta_{1}}$, there exists $\eta_{0}^{\prime}>0$ such that $N_{0}$ contains the set $X_{m_{0}<\eta_{0}^{\prime}} \cap X_{m_{1} \geq \eta_{1}}$. Thus by construction, the above identifications induce a compatible collection of isomorphisms of complexes

$$
\left(\Omega\left(\bar{U}_{0} \cap U_{1}, \partial U_{0} \cap U_{1}\right), d\right) \simeq\left(\Omega\left(X_{m_{0} \geq \eta_{0}} \cap X_{m_{1}>\eta_{1}}, X_{m_{0}=\eta_{0}} \cap X_{m_{1}>\eta_{1}}\right), d\right) .
$$


In what follows, we will use this reinterpretation of morphisms of $O p e n(X)$.

4.6. Morse theory. In the previous discussion, we have constructed a dg functor

$$
P: \operatorname{Open}(X) \rightarrow \operatorname{Sh}(X)
$$

such that $P$ identified $S h(X)$ as a triangulated envelope of $O p e n(X)$. In this section, using Morse theory we define an $A_{\infty}$-category $\operatorname{Mor}(X)$ and an $A_{\infty}$-functor

$$
M: \operatorname{Open}(X) \rightarrow \operatorname{Mor}(X)
$$

which is a quasi-equivalence.

In this section, to simplify the exposition we assume here that $X$ is oriented, and leave the general case to the reader.

4.6.1. Manifolds with corners. We begin by recalling some standard material from Morse theory. We first discuss some general facts for an arbitrary open subset $W \subset X$, then specialize to the case where the closure $\bar{W} \subset X$ is a manifold with corners.

Let $W \subset X$ be an open subset. Let $f: W \rightarrow \mathbb{R}$ be a function which extends to a small neighborhood of the closure $\bar{W} \subset X$ such that all critical points of the extension are nondegenerate and lie in $W$. Let $C r(f) \subset W$ denote the set of critical points, and let $i(x)$ denote the index of $x \in C r(f)$. Our convention is that a local minimum has index 0 , and a local maximum has index $\operatorname{dim} X$.

Fix a Riemannian metric $g$ on $X$, and let $\nabla f$ denote the gradient vector field. Let $\tilde{W} \subset W \times \mathbb{R}$ be a maximal domain for the the gradient flow $\psi_{t}: \tilde{W} \rightarrow W$. For each $w \in W$, the fiber of $\tilde{W}$ above $w$ is an open (possibly unbounded) interval. For each $x \in C r(f)$, define the stable and unstable manifolds

$$
W_{x}^{-}=\left\{w \in W \mid \lim _{t \rightarrow+\infty} \psi_{t}(w)=x\right\}, \quad W_{x}^{+}=\left\{w \in W \mid \lim _{t \rightarrow-\infty} \psi_{t}(w)=x\right\} .
$$

Implicit in the definition is that for $w \in W$ to lie in a stable or unstable manifold, the fiber of $\tilde{W}$ above $w$ contains the appropriate half-line. The stable manifold $W_{x}^{-}$and unstable manifold $W_{x}^{+}$are diffeomorphic to balls of dimension $i(x)$ and $\operatorname{dim} X-i(x)$ respectively. An orientation $\omega_{x}^{-}$for the stable manifold $W_{x}^{-}$and an orientation $\omega_{x}^{+}$for the unstable manifold $W_{x}^{+}$are said to be compatible if at $x$ the composite orientation $\omega_{x}^{-} \wedge \omega_{x}^{+}$coincides with the ambient orientation of $X$.

Now we specialize to the case when the closure $\bar{W} \subset X$ is a manifold with corners. To be precise, consider the quadrant

$$
\bar{Q}=\left\{\left(x_{1}, \ldots, x_{n}\right) \in \mathbb{R}^{n} \mid x_{1} \geq 0, x_{2} \geq 0\right\} .
$$

We assume that for each $w \in \bar{W} \subset X$, there is an open neighborhood $N(w) \subset X$, an open set $U \subset \mathbb{R}^{n}$, and a $C^{1}$ diffeomorphism $\psi: N(w) \rightarrow U$ such that

$$
\psi(\bar{W} \cap N(w))=\bar{Q} \cap U .
$$

Furthermore, we assume that there are two smooth transverse hypersurfaces $H_{0}, H_{1}$ $\subset X$ such that $\partial W \subset H_{0} \cup H_{1}$.

We will need the following notion of when a function $f$ on a manifold with corners $\bar{W} \subset X$ and a Riemannian metric $g$ on $X$ are compatible.

Definition 4.6.1. A pair $(f, g)$, where $f$ is a function on a neighborhood of $\bar{W}$ and $g$ is a Riemannian metric on $X$, is said to be directed if $(f, g)$ is Morse-Smale, and the gradient vector field $\nabla f$ is inward pointing along $H_{0}$ and outward pointing along $H_{1}$. 
With the above set-up, if we have a directed pair $(f, g)$, then for each $x \in C r(f)$, the closures of the stable and unstable manifolds satisfy

$$
\bar{W}_{x}^{-} \cap H_{1}=\emptyset, \quad \bar{W}_{x}^{+} \cap H_{0}=\emptyset .
$$

4.6.2. Morse moduli spaces. We next recall the moduli space of gradient trees from Fukaya-Oh [9].

A based metric ribbon tree is a quadruple $\left(T, i, v_{0}, \lambda\right)$ of the following data. First, $T$ is a finite tree with $d+1$ end vertices and no vertex containing exactly two edges. Second, $i: T \rightarrow D \subset \mathbb{R}^{2}$ is an embedding of $T$ in the closed unit disk such that the $d+1$ end vertices are precisely the intersection $i(T) \cap \partial D$. Third, $v_{0}$ is a distinguished end vertex of $T$. We refer to $v_{0}$ as the root vertex and the other $d$ end vertices as the leaf vertices. An edge $e \subset T$ is called an interior edge if $e$ does not contain an end vertex; otherwise $e$ is called an exterior edge. Finally, $\lambda$ is a function which assigns to every interior edge $e_{i n} \subset T$ a positive length $\lambda\left(e_{i n}\right) \in \mathbb{R}_{+}$. Two based metric ribbon trees are to be considered equivalent if there is an isotopy of the closed disk which identifies all of the data.

Fix the orientation of the edges of $T$ such that all arrows point in the direction of minimal paths from the leaf vertices to the root vertex. The left and right sides of an edge refer to the components of the complement $D \backslash T$ which are respectively to the left and right of the edge with respect to the orientation of the edge. Label the $d+1$ components of the complement $D \backslash T$ with elements of $\mathbb{Z} /(d+1) \mathbb{Z}$ in counterclockwise order starting with 0 for the component to the left of the edge terminating at the root vertex $v_{0}$. For an edge $e \subset T$, let $\ell(e)$ and $r(e)$ denote the labelings of the left and right sides of $e$.

For $i \in \mathbb{Z} /(d+1) \mathbb{Z}$, let $U_{i} \subset X$ be an open subset with boundary $\partial U_{i} \subset X$ a smooth hypersurface. Suppose that the boundaries $\partial U_{i}$ form a transverse collection of hypersurfaces. Let $f_{i}: U_{i} \rightarrow \mathbb{R}$ be a function which extends to a small neighborhood of the closure $\bar{U}_{i} \subset X$. The difference $f_{i+1}-f_{i}$ is a function on the intersection $U_{i} \cap U_{i+1}$ which extends to a small neighborhood of the closure $\bar{U}_{i} \cap U_{i+1} \subset X$. Suppose the critical points of the extension of $f_{i+1}-f_{i}$ are nondegenerate and lie in $U_{i} \cap U_{i+1}$. Suppose that we have chosen a Riemannian metric $g$ on $X$ such that each pair $\left(f_{i+1}-f_{i}, g\right)$ is directed. In other words, each gradient vector field $\nabla f_{i+1}-\nabla f_{i}$ points inward along the hypersurface $\partial U_{i+1}$ and outward along the hypersurface $\partial U_{i}$.

With this set-up, a gradient tree is a pair $\left(\left(T, i, v_{0}, \lambda\right), \tau\right)$ consisting of a metric ribbon tree $\left(T, i, v_{0}, \lambda\right)$ and a continuous map $\tau: T \rightarrow X$ such that the following holds:

(1) For each end vertex $v \in T$ and the unique exterior edge $e_{e x t} \in T$ containing it, we have

$$
\tau(v) \in C r\left(U_{r\left(e_{e x t}\right)} \cap U_{\ell\left(e_{e x t}\right)}, f_{r\left(e_{e x t}\right)}-f_{\ell\left(e_{e x t}\right)}\right) .
$$

(2) For each interior edge $e_{i n} \subset T$, after making the identification $e_{i n} \simeq$ $\left[0, \lambda\left(e_{i n}\right)\right]$, we have

$$
\left.\tau^{\prime}\right|_{e_{i n}}=-\nabla\left(f_{\ell\left(e_{i n}\right)}-f_{r\left(e_{i n}\right)}\right) .
$$

(3) For each exterior edge $e_{\text {ext }} \subset T$, after making the identification $e_{\text {ext }} \simeq$ $(-\infty, 0]$, we have

$$
\left.\tau^{\prime}\right|_{e_{e x t}}=-\nabla\left(f_{\ell\left(e_{e x t}\right)}-f_{r\left(e_{e x t}\right)}\right) .
$$


Note that a single gradient tree alone contains the information of the images of the vertices and the oriented gradient lines which are the images of the edges.

For each $i \in \mathbb{Z} /(d+1) \mathbb{Z}$, fix a critical point $x_{i} \in C r\left(U_{i} \cap U_{i+1}, f_{i+1}-f_{i}\right)$. After a small perturbation of the data, the moduli space of gradient trees

$$
\mathcal{M}\left(T ; f_{0}, \ldots, f_{d} ; x_{0}, \ldots, x_{d}\right)
$$

with specified critical points is a manifold of dimension

$$
\sum_{i \in \mathbb{Z} /(d+1) \mathbb{Z}} i\left(x_{i}\right)-d \operatorname{dim} X+d-2 .
$$

Orientations of the stable manifolds of the differences $f_{i+1}-f_{i}$ induce a canonical orientation of the moduli space. For example, for $d=1$, a single edge is the only based ribbon metric tree, and the moduli space $\mathcal{M}\left(T ; f_{0}, f_{1} ; x_{0}, x_{1}\right)$ is the space of trajectories from $x_{0}$ to $x_{1}$ with orientations.

4.6.3. Morse $A_{\infty}$-category. Following Fukaya-Oh [9], we define an $A_{\infty}$-category $\operatorname{Mor}(X)$ as follows. As with $\operatorname{Open}(X)$, the objects of $\operatorname{Mor}(X)$ are pairs $\mathfrak{U}=(U, m)$, where $U \subset X$ is an open set and $m: X \rightarrow \mathbb{R}_{\geq 0}$ is a defining function for the complement $X \backslash U$. To this data, we associate the function $f: U \rightarrow \mathbb{R}$ defined by $f=\log m$.

To define the morphisms from an object $\mathfrak{U}_{0}=\left(U_{0}, m_{0}\right)$ to an object $\mathfrak{U}_{1}=$ $\left(U_{1}, m_{1}\right)$, we will associate to them a directed pair and assign its Morse complex. To ensure that we may find a directed pair, we must refine the procedure summarized in Section 4.5

First, fix a Whitney stratification $\mathcal{S}_{0}$ of $X$ compatible with the boundary $\partial U_{0} \subset$ $X$, and let $\Lambda_{\mathcal{S}_{0}} \subset T^{*} X$ be the conical conormal set associated to $\mathcal{S}_{0}$. By Lemma3.2.4, there is $\bar{\eta}_{1}>0$ such that there are no $\Lambda_{0}$-critical values of $m_{1}$ in the open interval $\left(0, \bar{\eta}_{1}\right)$.

Next, choose $\eta_{1} \in\left(0, \bar{\eta}_{1}\right)$, fix the Whitney stratification $\mathcal{S}_{\eta_{1}}$ of $X$ given by the hypersurface $X_{m_{1}=\eta_{1}}$ and its complement, and let $\Lambda_{\mathcal{S}_{\eta_{1}}} \subset T^{*} X$ be the conical conormal set associated to $\mathcal{S}_{\eta_{1}}$. By Lemma 3.2.4, there is $\bar{\eta}_{0}>0$ such that there are no $\Lambda_{\mathcal{S}_{\eta_{1}}}$-critical values of $m_{0}$ in the open interval $\left(0, \bar{\eta}_{0}\right)$.

Now consider the critical points of the function $m_{0} \times m_{1}: X \rightarrow \mathbb{R}^{2}$. By definition, critical points of $m_{0} \times m_{1}$ are points $x \in X$ such that $d m_{0}(x)$ and $d m_{1}(x)$ are colinear. Note that for points of $U_{0} \cap U_{1}$, this is equivalent to $d f_{0}$ and $d f_{1}$ being colinear. By construction, there are no critical values of $m_{0} \times m_{1}$ in the interval $\left(0, \bar{\eta}_{0}\right) \times\left\{\eta_{1}\right\} \subset \mathbb{R}^{2}$. In other words, all critical points of $m_{0} \times m_{1}$ which lie on the hypersurface $X_{m_{1}=\eta_{1}}$ lie in the compact region $X_{m_{0} \geq \bar{\eta}_{0}, m_{1}=\eta_{1}}$. Thus we may choose $\bar{\epsilon}_{0}>0$ such that for any $\epsilon_{0} \in\left(0, \bar{\epsilon}_{0}\right)$ and any point $x \in X_{m_{1}=\eta_{1}}$, where $d m_{0}$ and $d m_{1}$ are colinear, we have

$$
\epsilon_{0}\left|\frac{d f_{0}(x)}{d f_{1}(x)}\right|<1 .
$$

Here the fraction notation reflects the fact that the two covectors are colinear and so differ by a scalar. Note in particular that $d f_{1}(x) \neq 0$ on $X_{m_{1}=\eta_{1}}$. This bound will guarantee that we may choose a Riemannian metric on $X$ such that the gradient $\nabla f_{1}-\epsilon_{0} \nabla f_{0}$ is inward pointing along $X_{m_{1}=\eta_{1}}$.

Next, we need to choose $\eta_{0}>0$ small enough so that we may choose a Riemannian metric on $X$ such that the gradient $\nabla f_{1}-\epsilon_{0} \nabla f_{0}$ is outward pointing along $X_{m_{0}=\eta_{0}}$. Note that the values of $d f_{1}$ along the compact hypersurfaces $X_{m_{1}=\eta_{1}}$ are 
bounded. Furthermore, the values of $d f_{0}$ along the compact hypersurface $X_{m_{0}=\eta_{0}}$ tend uniformly to infinity as $\eta_{0}$ tends to zero. Thus for any $\eta_{0}>0$ small enough and any point $x \in X_{m_{0}=\eta_{0}}$ where $d m_{0}$ and $d m_{1}$ are colinear, we have

$$
\left|\frac{d f_{1}(x)}{\epsilon_{0} d f_{0}(x)}\right|<1 \text {. }
$$

In conclusion, for a sequence of sufficiently small choices $\eta_{1}>0$, then $\epsilon_{0}>0$, and then $\eta_{0}>0$, we have the following result.

Lemma 4.6.2. There is a Riemannian metric $g$ on $X$ such that $\left(f_{1}-\epsilon_{0} f_{0}, g\right)$ is a directed pair on the manifold with corners $X_{m_{0} \geq \eta_{0}, m_{1} \geq \eta_{1}}$. The set of such metrics is open and convex.

Proof. The construction of the metric can be done locally using the bounds of the above procedure. The conditions on the metric are open and convex.

Finally, we choose small perturbations of our functions and metric, and define the space of morphisms to be the graded vector space generated by critical points

$$
\operatorname{hom}_{M o r(X)}\left(\mathfrak{U}_{0}, \mathfrak{U}_{1}\right)=\mathbb{C}\left\{C r\left(X_{m_{0}>\eta_{0}} \cap X_{m_{1}>\eta_{1}}, f_{1}-\epsilon_{0} f_{0}\right)\right\} .
$$

The differential counts the oriented number of points in the moduli spaces of gradient trees

$$
m_{M o r(X)}^{1}\left(x_{0}\right)=\sum_{T} \sum_{x_{1}} \operatorname{deg} \mathcal{M}\left(T ; \epsilon_{0} f_{0}, f_{1} ; x_{0}, x_{1}\right) \cdot x_{1}
$$

with $T$ the interval $[-1,1]$.

Lemma 4.6.3. We have that $m_{M o r(X)}^{1}$ is well-defined and satisfies $\left(m_{M o r(X)}^{1}\right)^{2}=$ 0 .

Proof. Fix critical points $x_{0}, x_{1}$. Both assertions are implied by the claim that any sequence of gradient trajectories

$$
\tau_{n}:[-1,1] \rightarrow X_{m_{0}>\eta_{0}, m_{1}>\eta_{1}}
$$

in the moduli space $\mathcal{M}\left([-1,1] ; \epsilon_{0} f_{0}, f_{1} ; x_{0}, x_{1}\right)$ has a subsequence which converges to a map

$$
\tau_{\infty}:[-1,1] \rightarrow X_{m_{0}>\eta_{0}, m_{1}>\eta_{1}} .
$$

The claim with $\operatorname{deg} x_{1}=\operatorname{deg} x_{0}+1$ proves the first assertion. The claim with $\operatorname{deg} x_{1}=\operatorname{deg} x_{0}+2$ implies that the boundary of the moduli space in this case is precisely the usual moduli space of broken trajectories which calculates $\left(m_{M \operatorname{or}(X)}^{1}\right)^{2}$. This proves the second assertion.

To prove the claim, observe that the image set $\tau_{n}([-1,1])$ can never approach the boundary

$$
\partial X_{m_{0} \geq \eta_{0}, m_{1} \geq \eta_{1}}=\left(X_{m_{0}=\eta_{0}} \cap X_{m_{1} \geq \eta_{1}}\right) \cup\left(X_{m_{1} \geq \eta_{0}} \cap X_{m_{1}=\eta_{1}}\right) .
$$

This follows from our assumptions on the behavior of the gradient vector field of $f_{1}-\epsilon f_{0}$ along the boundary (inward and outward pointing).

By construction, the morphism complex $\left(\operatorname{hom}_{M o r(X)}\left(\mathfrak{U}_{0}, \mathfrak{U}_{1}\right), \mu_{M o r(X)}^{1}\right)$ calculates the relative cohomology

$$
H^{*}\left(X_{m_{0} \geq \eta_{0}} \cap X_{m_{1}>\eta_{1}}, X_{m_{0}=\eta_{0}} \cap X_{m_{1}>\eta_{1}}\right) .
$$


To define the higher compositions, for a finite collection of objects we must follow the above procedure sequentially. What we need is summarized in the following definition.

Definition 4.6.4. Consider a collection of pairs $\left(U_{i}, f_{i}\right)$, where $U_{i} \subset X$ is an open subset with boundary $\partial U_{i}$ a smooth hypersurface and $f_{i}: U_{i} \rightarrow \mathbb{R}$ is a function indexed by $i \in \mathbb{Z} /(d+1) \mathbb{Z}$. The collection is said to be transverse if there is a Riemannian metric $g$ on $X$ such that the following holds. For $i \in \mathbb{Z} /(d+1) \mathbb{Z}$, the hypersurfaces $\partial U_{i}$ and $\partial U_{i+1}$ are transverse, and $\left(f_{i+1}-f_{i}, g\right)$ is a directed pair on $U_{i} \cap U_{i+1}$.

In Section 6] we will carefully explain in the context of the Fukaya category of $T^{*} X$ how to arrive at such a collection. The procedure described there is modestly more complicated, but strictly contains what is needed here. Therefore we will not pursue further details, but only mention the following salient points.

Given a collection of objects indexed by $i \in \mathbb{Z} /(d+1) \mathbb{Z}$, for any sequence of sufficiently small choices $\epsilon_{i}>0$ and $\eta_{i}>0$, one may arrange for the perturbed boundaries $X_{m_{i}=\eta_{i}}$ to form a transverse collection. Furthermore, one may sequentially obtain bounds on the differentials $d f_{i}$ along the perturbed boundaries. Together this allows one to find dilations and a Riemannian metric on $X$ such that all dilated pairs are directed. After performing small perturbations, the higher composition maps count the oriented number of points in the moduli spaces of gradient trees

$$
m_{M o r(X)}^{d}\left(x_{0}, \ldots, x_{d-1}\right)=\sum_{T} \sum_{x_{d}} \operatorname{deg} \mathcal{M}\left(T ; \epsilon_{0} f_{0}, \ldots, \epsilon_{d-1} f_{d-1}, f_{d} ; x_{0}, \ldots, x_{d}\right) \cdot x_{d} .
$$

In the following section, we will apply homological perturbation theory to verify the following.

Proposition 4.6.5. The maps $\mu_{M o r(X)}^{d}$ are well defined and satisfy the $A_{\infty}$-quadratic composition rule.

In conclusion, it is worth commenting about the choices involved in the construction of $\operatorname{Mor}(X)$. For a collection of objects indexed by $i \in \mathbb{Z} /(d+1) \mathbb{Z}$, there are the small choices of constants $\eta_{i}>0$ to obtain smooth boundaries and $\epsilon_{i}>0$ to dilate functions. These may be organized into a contractible "fringed set" as discussed in Section 5.2. In addition, there is the choice of a Riemannian metric to obtain directed pairs. While not a perturbation in any sense, such metrics form a convex set. Finally, there are the small perturbations of the functions and metric. This is no different from the standard context.

4.6.4. From differential forms to Morse theory. Following Kontsevich-Soibelman [25, here we apply the formalism of homological perturbation theory to prove Proposition 4.6.5 and obtain an $A_{\infty}$-functor

$$
M: \operatorname{Open}(X) \rightarrow \operatorname{Mor}(X)
$$

which is a quasi-equivalence. We will apply the formalism in the special case of an idempotent. The construction of the idempotent and the homotopy is completely analogous to that of Kontsevich-Soibelman: one composes the limit operators of Harvey-Lawson [15] with a smoothing operator. To explain this, we return to the general context of a submanifold with corners $\bar{W} \subset X$ and boundary hypersurfaces $H_{0}, H_{1} \subset \partial W$ with which we began this section. 
Let $D^{\prime}\left(W, H_{0}\right)$ denote the space of currents dual to the space of differential forms $\Omega\left(W, H_{1}\right)$. There are two simple ways to obtain elements of $D^{\prime}\left(W, H_{0}\right)$. First, we have the inclusion

$$
i: \Omega\left(W, H_{0}\right) \rightarrow D^{\prime}\left(W, H_{0}\right)
$$

defined by taking the wedge product of forms and integrating. Second, any oriented closed submanifold $V \subset W$ satisfying $\bar{V} \cap H_{0}=\emptyset$ defines an element

$$
[V] \in D^{\prime}\left(W, H_{0}\right)
$$

by integration. In particular, for each $x \in C r(f)$, the unstable manifold $W_{x}^{+}$with a given orientation defines a current $\left[W_{x}^{+}\right] \in D^{\prime}\left(W, H_{0}\right)$. Similarly, the stable manifold $W_{x}^{-}$with a given orientation defines a current $\left[W_{x}^{-}\right] \in D^{\prime}\left(W, H_{1}\right)$.

In this context, the main result of Harvey-Lawson takes the following form. Define the linear operator $p: \Omega\left(W, H_{0}\right) \rightarrow D^{\prime}\left(W, H_{0}\right)$ by the kernel

$$
\mathcal{P}=\sum_{x \in C r(f)}\left[W_{x}^{-}\right] \times\left[W_{x}^{+}\right],
$$

where the stable and unstable manifolds are given compatible orientations. Define the homotopy operator $h: \Omega\left(W, H_{0}\right) \rightarrow D^{\prime}\left(W, H_{0}\right)$ by the kernel

$$
\mathcal{H}=\bigcup_{0 \leq t<+\infty}\left[\Gamma_{\psi_{t}}\right]
$$

where $\Gamma_{\psi_{t}} \subset X \times X$ denotes the graph of the gradient flow $\psi_{t}$. Then we have the equation of operators

$$
p-i=d h+h d \text {. }
$$

Now to obtain an honest idempotent $\pi$ and homotopy operator $T$ on $\Omega\left(W, H_{0}\right)$, we need only compose with a smoothing operator $D^{\prime}\left(W, H_{0}\right) \rightarrow \Omega\left(W, H_{0}\right)$. The details of this are no different from the case considered by Kontsevich-Soibelman.

By applying the formalism of homological perturbation theory and recognizing that it coincides with counting gradient trees, we see that Proposition 4.6.5 must hold and obtain an $A_{\infty}$-functor

$$
M: \operatorname{Open}(X) \rightarrow \operatorname{Mor}(X)
$$

which is a quasi-equivalence.

\section{The Fukaya CATEgory}

The Fukaya $A_{\infty}$-category $F u k(M)$ of a symplectic manifold $M$ is a quantization of the Lagrangian intersection theory of $M$. Roughly speaking, its objects are Lagrangian submanifolds and its morphisms are generated by intersection points of the Lagrangians. Its composition maps are defined by choosing a compatible almost complex structure and by counting holomorphic polygons with boundary lying on the Lagrangians 2 For example, for intersection points $p_{0} \in L_{0} \cap L_{1}$ and $p_{1} \in L_{1} \cap L_{2}$, the coefficient of $p_{2} \in L_{2} \cap L_{0}$ in the product $p_{0} \cdot p_{1}$ is the number of holomorphic maps to $M$ from a disk with three marked boundary points mapping to the intersection points and with the arcs between them mapping to the Lagrangians.

To this coarse description there are many details, refinements, and specializations for various settings. In this paper, we will use a composite picture of the treatments from Eliashberg-Givental-Hofer [7, Fukaya-Oh-Ohta-Ono [10], and Seidel [33. Our

\footnotetext{
${ }^{2}$ Properly, we should say "pseudoholomorphic," but we omit the prefix throughout.
} 
symplectic manifold is the cotangent bundle $M=T^{*} X$ of a compact real analytic manifold $X$. We equip $T^{*} X$ with the exact symplectic form $\omega=d \theta$, where $\theta$ is the canonical one-form

$$
\left.\theta(v)\right|_{(x, \xi)}=\xi\left(\pi_{*} v\right)
$$

with $\pi: T^{*} X \rightarrow X$ the standard projection. For any choice of Riemannian metric on $X$, the associated Levi-Civita connection provides a compatible almost complex structure on $T^{*} X$, along with a canonical Riemannian (Sasaki) metric on $T^{*} X$. We will also consider a mild variation of these structures as described in Section 5.1 .3 below.

In what follows, we focus on the aspects of our situation which deviate from what is by now standard in the subject. All of these differences stem from the fact that we will allow closed but noncompact Lagrangians.

We often use the following notation: given a space $Y$, a function $g: Y \rightarrow \mathbb{R}$, and $r \in \mathbb{R}$, we write $Y_{g=r}$ for the subset $\{y \in Y \mid g(y)=r\}$, and similarly for inequalities.

\subsection{Basics of $T^{*} X$.}

5.1.1. Compactification. Consider the bundle $J_{\geq 0}^{1}(X)=T^{*} X \times \mathbb{R}_{\geq 0}$ of 1-jets of nonnegative functions on $X$, and let $J_{\geq 0}^{1}(X)^{\prime}=J_{\geq 0}^{1}(X) \backslash(X \times\{0\})$ be the complement of the zero section. The multiplicative group $\mathbb{R}_{+}$acts freely on $J_{\geq 0}^{1}(X)^{\prime}$ by dilations. The quotient

$$
\bar{T}^{*} X=J_{\geq 0}^{1}(X)^{\prime} / \mathbb{R}_{+},
$$

equipped with the obvious projection $\bar{\pi}: \bar{T}^{*} X \rightarrow X$, provides a relative compactification of $\pi: T^{*} X \rightarrow X$. We have the canonical inclusion $T^{*} X \hookrightarrow \bar{T}^{*} X$ which sends a covector $\xi$ to the class of 1 -jets $[\xi, 1]$, and we refer to this inclusion implicitly whenever we consider $T^{*} X$ as a subset of $\bar{T}^{*} X$. The divisor at infinity

$$
T^{\infty} X=\bar{T}^{*} X \backslash T^{*} X
$$

consists of the class of 1 -jets of the form $[\xi, 0]$ with $\xi$ a nonzero covector.

Let $\mathcal{O}(-1)$ denote the tautological $\mathbb{R}_{+}$-principal bundle $J_{>0}^{1}(X)^{\prime} \rightarrow \bar{T}^{*} X$ with fiber $\mathbb{R}_{+} \cdot(\xi, r)$ at the point $[\xi, r]$. The restriction of $\mathcal{O}(-1)$ to the open subset $T^{*} X$ is canonically trivialized by the section $[\xi, r] \mapsto(\xi / r, 1)$. Let $\mathcal{O}(-1)_{\infty}$ denote the restriction of $\mathcal{O}(-1)$ to the divisor at infinity $T^{\infty} X$. A choice of trivialization of $\mathcal{O}(-1)_{\infty}$ is equivalent to a choice of (co-)sphere subbundle $S^{*} X \subset T^{*} X$ and provides a canonical identification

$$
T^{\infty} X \simeq S^{*} X .
$$

In fact, it is always possible to trivialize $\mathcal{O}(-1)$ itself over all of $\bar{T}^{*} X$. For example, if we choose a Riemannian metric on $X$, then we have the section

$$
[\xi, r] \mapsto(\hat{\xi}, \hat{r}) \text {, where }|\hat{\xi}|^{2}+\hat{r}^{2}=1 .
$$

This identifies $\bar{T}^{*} X$ with the closed unit disk bundle and $T^{\infty} X$ with its unit sphere bundle. Note that such a trivialization cannot be made equal to the canonical trivialization of $\mathcal{O}(-1)$ over the open subset $T^{*} X$.

By working with a spherical compactification rather than a projective compactification, we pay the price for dealing with a manifold with boundary. But we choose this approach because we will encounter objects on $T^{\infty} X$ which are not invariant under the antipodal involution. 
The pull-back of $\theta$ to $J_{\geq 0}^{1}(X)$ descends to a one-form $\bar{\theta}$ on $\bar{T}^{*} X$ with values in the $\mathbb{R}$-line bundle $L(1)$ associated to the $\mathbb{R}_{+}$-principal bundle dual to $\mathcal{O}(-1)$. The restriction $\theta^{\infty}=\left.\bar{\theta}\right|_{T^{\infty} X}$ is an $L(1)$-valued contact form on $T^{\infty} X$. The canonical trivialization of $\mathcal{O}(-1)$ over the open subset $T^{*} X$ identifies the restriction $\left.\bar{\theta}\right|_{T^{*} X}$ with the original one-form $\theta$. By choosing a trivialization of $\mathcal{O}(-1)_{\infty}$, we may consider $\theta^{\infty}$ as an honest contact form. Equivalently, by choosing a sphere bundle $S^{*} X \subset T^{*} X$, we may identify $\theta^{\infty}$ with the restriction of $\theta$ to $S^{*} X$. If we do not fix such identifications, we still have a well-defined contact structure $\operatorname{ker}\left(\theta^{\infty}\right) \subset T T^{\infty} X$ and a well-defined notion of positive normal direction. This positive direction is an example of a structure on $T^{\infty} X$ which is not invariant under the antipodal map.

5.1.2. Geodesic flow. Given a function $H: T^{*} X \rightarrow \mathbb{R}$, we have the Hamiltonian vector field $v_{H}$ defined by

$$
d H(v)=\omega\left(v, v_{H}\right) .
$$

When possible, integrating $v_{H}$ provides a Hamiltonian isotopy $\varphi_{H, t}: T^{*} X \rightarrow T^{*} X$.

A Riemannian metric on $X$ defines a Riemannian (Sasaki) metric on $T^{*} X$, and thus an identification $T^{*}\left(T^{*} X\right) \simeq T\left(T^{*} X\right)$. The canonical one-form $\theta$ on $T^{*} X$ corresponds to the geodesic vector field $v_{\theta}$. On the complement of the zero section $T^{*} X \backslash X$, we have the normalized geodesic vector field $\hat{v}_{\theta}=v_{\theta} /\left|v_{\theta}\right|$. It is the Hamiltonian vector field for the length function $H: T^{*} X \backslash X \rightarrow \mathbb{R}$ given by $H(x, \xi)=|\xi|$. We write $\gamma_{t}: T^{*} X \backslash X \rightarrow T^{*} X \backslash X$ for the normalized geodesic flow for time $t$ associated to $\hat{v}_{\theta}$. By definition, if we identify a covector $(x, \xi) \in T^{*} X$ with a vector $(x, v) \in T X$, then we have the identity

$$
\gamma_{t}(x, v)=\exp _{x, t}(\hat{v})_{*}(v),
$$

where $\hat{v}=v /|v|$, the map $\exp _{x, t}: T_{x} X \rightarrow X$ denotes the exponential flow from the point $x$ for time $t$, and the asterisk subscript indicates the derivative (pushforward). Since $v_{\theta}$ grows at infinity, its flow does not have a well-defined limit. But $\hat{v}_{\theta}$ extends to give a Reeb flow on the contact manifold at infinity $T^{\infty} X$.

A function $H: T^{*} X \rightarrow \mathbb{R}$ is said to be controlled if there is a compact set $K \subset T^{*} X$ such that outside of $K$ we have $H(x, \xi)=|\xi|$. The corresponding Hamiltonian isotopy $\varphi_{H, t}: T^{*} X \rightarrow T^{*} X$ equals the normalized geodesic flow $\gamma_{t}$ outside of $K$. Note that for any controlled function $H$, the vector field $v_{H}$ may be integrated to a Hamiltonian isotopy $\varphi_{H, t}$, for all times $t$. Note as well that Hamiltonian flow by $|\xi|$ depends on the metric on $X$, but is independent of any choice of metric on $T^{*} X$.

5.1.3. Almost complex structures. To better control holomorphic disks in $T^{*} X$, it is useful to introduce an almost complex structure $J_{c o n}$ which near infinity is invariant under dilations.

Recall that a Riemannian metric on $X$ provides a canonical splitting

$$
T\left(T^{*} X\right) \simeq T_{b} \oplus T_{f},
$$

where $T_{b}$ denotes the horizontal base directions and $T_{f}$ the vertical fiber directions, along with a canonical isomorphism $j_{0}: T_{b} \stackrel{\sim}{\rightarrow} T_{f}$ of vector bundles. Thus we can define a compatible almost complex structure $J_{S a s}$ by the matrix

$$
J_{\text {Sas }}=\left(\begin{array}{cc}
0 & j_{0}^{-1} \\
-j_{0} & 0
\end{array}\right) \text {. }
$$


We refer to $J_{S a s}$ as the Sasaki almost complex structure, since by construction, the Sasaki metric is given by $g_{\text {Sas }}(U, V)=\omega\left(U, J_{\text {Sas }} V\right)$.

Fix a positive function $w: T^{*} X \rightarrow \mathbb{R}_{>0}$, and define a new compatible almost complex structure $J_{w}$ by the matrix

$$
J_{w}=\left(\begin{array}{cc}
0 & w^{-1} j_{0}^{-1} \\
-w j_{0} & 0
\end{array}\right) .
$$

Fix a local orthonormal frame $\left\{b_{i}, f_{i}\right\}_{i=1}^{\operatorname{dim} X}$ for $T_{b} \oplus T_{f}$ with respect to the Sasaki metric. Then with respect to the new metric $g_{w}(U, V)=\omega\left(U, J_{w} V\right)$, the lengths of the Sasaki frame take the form

$$
\left|b_{i}\right|_{g_{w}}=w^{1 / 2}, \quad\left|f_{i}\right|_{g_{w}}=w^{-1 / 2} .
$$

For concreteness, we specialize the construction by making a specific choice of the function $w$. Namely, fix positive constants $r_{0}, r_{1}>0$, and a bump function $b: \mathbb{R} \rightarrow \mathbb{R}$ such that $b(r)=0$ for $r<r_{0}$ and $b(r)=1$ for $r>r_{1}$. Fix a constant $\beta \in \mathbb{R}$, and set

$$
w(x, \xi)=|\xi|^{\beta b(|\xi|)},
$$

where as usual $|\xi|$ denotes the length of a covector with respect to the original metric on $X$. In particular, when $\beta=0$, we recover the original Sasaki almost complex structure $J_{\text {Sas }}$ and Sasaki metric $g_{\text {Sas }}$.

In what follows, we will restrict our attention to the choice $\beta=1$ and denote the almost complex structure by $J_{c o n}$ and corresponding metric by $g_{c o n}$. We will refer to $J_{c o n}$ as the conical almost complex structure, since near infinity $J_{c o n}$ is invariant under dilations and the lengths of our Sasaki frame take the form

$$
\left|b_{i}\right|_{g_{\text {con }}}=|\xi|^{1 / 2}, \quad\left|f_{i}\right|_{g_{c o n}}=|\xi|^{-1 / 2} .
$$

Thus near infinity, we have replaced the Sasaki geometry with a cone over the unit (co-)sphere bundle $S^{*} X$. To be precise, if $d s^{2}$ is the restriction of the Sasaki metric to $S^{*} X$, and for clarity we write $r$ for the length $|\xi|$, then near infinity we have

$$
g_{\text {con }}=r^{-1} d r^{2}+r d s^{2} \text {. }
$$

(Substituting $\tilde{r}=r^{1 / 2}$, one sees the familiar presentation of the metric of a cone.) It is straightforward to check that $g_{c o n}$ is complete and that the normalized geodesic flow $\gamma_{t}$ is an isometry.

We will confirm in Section 5.4 .3 that the almost complex structure $J_{\text {con }}$ provides compact moduli spaces of holomorphic disks in the circumstances under consideration. One can view the metric $g_{\text {con }}$ as being compatible with the compactification $\bar{T}^{*} X$ in the sense that near infinity, it treats base and angular fiber directions on equal footing: near infinity, the metrics on the level sets of $|\xi|$ are simply scaled by the factor $|\xi|^{1 / 2}$.

5.2. Lagrangians. Fix an analytic-geometric category $\mathcal{C}$.

Lemma 5.2.1. For any $\mathcal{C}$-subset $\bar{V} \subset \bar{T}^{*} X$, there exists $r>0$ such that $|\xi|$ has no critical points on $\bar{V} \cap T^{*} X$ for $|\xi| \geq r$.

Proof. The critical values of $1 /|\xi|$ are a discrete $\mathcal{C}$-subset of $\mathbb{R}$.

Lemma 5.2.2. Let $W$ be a compact space, and let $V \subset T^{*} X$ be a subset such that $|\xi|$ has no critical points on $V$ for $|\xi| \geq r$. Then any map $W \rightarrow V$ is homotopic in $V$ to a map $W \rightarrow V_{|\xi|<r}$. 
Proof. By the Thom isotopy lemma, we may use the gradient of $|\xi|$ to flow the image of the map $W \rightarrow V$.

A subset $V \subset T^{*} X$ is said to be conical if it is invariant under the action of $\mathbb{R}_{+}$ by fiberwise dilations.

Lemma 5.2.3. If $V$ is a conical $\omega$-isotropic subset of $T^{*} X$, then $\bar{V} \cap T^{\infty} X$ is a $\theta^{\infty}$-isotropic subset of $T^{\infty} X$.

Proof. The one-form $\theta$ may be obtained from the symplectic form $\omega$ by contracting with the Liouville vector field $v_{\theta}$. The action of $\mathbb{R}_{+}$by dilations is generated by $v_{\theta}$.

As long as we assume that $\bar{V} \subset \bar{T}^{*} X$ is a $\mathcal{C}$-subset, we have the following very general result.

Lemma 5.2.4. If $\bar{V} \subset \bar{T}^{*} X$ is a $\mathcal{C}$-subset such that $\bar{V} \cap T^{*} X$ is an $\omega$-isotropic subset of $T^{*} X$, then $\bar{V} \cap T^{\infty} X$ is a $\theta^{\infty}$-isotropic subset of $T^{\infty} X$.

Proof. Let $\mathcal{N}^{\infty} X$ be the family with general fiber $\bar{T}^{*} X$ and special fiber the normal cone $N^{\infty} X$ of $\bar{T}^{*} X$ along the divisor $T^{\infty} X$. Let $\bar{C} \subset N^{\infty} X$ be the limit of $\bar{V}$ under specialization in the family $\mathcal{N}^{\infty} X$. (See [20], pp. 185-187, for an exposition of the normal cone and the specialization of subsets.) By construction, $\bar{C}$ is a conical subset satisfying $\bar{C} \cap T^{\infty} X=\bar{V} \cap T^{\infty} X$.

We claim that $\bar{C}$ is $\omega$-isotropic. (The normal cone $N^{\infty} X$ inherits a well-defined $\omega$-isotropic distribution.) If we can show this, then we are done by the previous lemma. To see this, choose a Whitney stratification of $\mathcal{N}^{\infty} X$ compatible with $\bar{C}$; this is possible since $\bar{V}$ is a $\mathcal{C}$-subset. Then the Whitney condition and the fact that being $\omega$-isotropic is a closed condition together imply the assertion: the tangent spaces of the limit $\bar{C}$ are contained in the limits of the $\omega$-isotropic tangent spaces of $\bar{V}$.

We will need to separate $\theta^{\infty}$-isotropic subsets of $T^{\infty} X$ using the normalized geodesic flow (Reeb flow). To organize this, we use a variant of the notion of a fringed set from [12]. To define what a fringed set $R_{d+1} \subset \mathbb{R}_{+}^{d+1}$ is, we proceed inductively. A fringed set $R_{1} \subset \mathbb{R}_{+}$is any interval of the form $(0, r)$ for some $r>0$. A fringed set $R_{d+1} \subset \mathbb{R}_{+}^{d+1}$ is a subset satisfying the following:

(1) $R_{d+1}$ is open in $\mathbb{R}_{+}^{d+1}$.

(2) Under the projection $\pi: \mathbb{R}^{d+1} \rightarrow \mathbb{R}^{d}$, the image $\pi\left(R_{d+1}\right)$ is a fringed set.

(3) If $\left(r_{1}, \ldots, r_{d}, r_{d+1}\right) \in R_{d+1}$, then $\left(r_{1}, \ldots, r_{d}, r_{d+1}^{\prime}\right) \in R_{d+1}$ for all $0<$ $r_{d+1}^{\prime}<r_{d+1}$.

It is easy to check that fringed sets as defined here are contractible.

Lemma 5.2.5. For $i=0, \ldots, d$, let $V_{i}^{\infty} \subset T^{\infty} X$ be $\theta^{\infty}$-isotropic compact subsets. Then there is a fringed set $R_{d+1} \subset \mathbb{R}^{d+1}$ such that for $\left(\delta_{0}, \ldots, \delta_{d}\right) \in R_{d+1}$, the normalized geodesic flow (Reeb flow) separates the subsets:

$$
\gamma_{\delta_{i}}\left(V_{i}^{\infty}\right) \cap \gamma_{\delta_{j}}\left(V_{j}^{\infty}\right)=\emptyset \text {, for } i \neq j .
$$

Proof. We prove the assertion by induction. For $d=0$, there is nothing to prove. Suppose we know the assertion for $d-1$ and seek to establish it for $d$. For 
$\left(\delta_{0}, \ldots, \delta_{d-1}\right) \in R_{d}$, consider the $\theta^{\infty}$-isotropic subset

$$
V_{<d}^{\infty}=\bigcup_{i<d} \gamma_{\delta_{i}}\left(V_{i}^{\infty}\right)
$$

It suffices to show that there is $\delta_{d}=\delta_{d}\left(\delta_{0}, \ldots, \delta_{d-1}\right)>0$ such that for all $0<\delta_{d}^{\prime}<$ $\delta_{d}$, we have

$$
\gamma_{\delta_{d}^{\prime}}\left(V_{d}^{\infty}\right) \cap V_{<d}^{\infty}=\emptyset .
$$

Suppose this were not true. Then by the curve selection lemma (Proposition 3.2.5), there is a $C^{1} \operatorname{map} \alpha:[0,1) \rightarrow V_{d}^{\infty}$ such that for all $t \in(0,1)$,

$$
\gamma_{t}(\alpha(t)) \in \gamma_{t}\left(V_{d}^{\infty}\right) \cap V_{<d}^{\infty} .
$$

In particular, $\gamma_{t}(\alpha(t))$ lies in the $\theta^{\infty}$-isotropic subset $V_{<d}^{\infty}$. But we calculate

$$
\left.\frac{d}{d t} \gamma_{t}(\alpha(t))\right|_{t=0}=\gamma_{0}^{\prime}(\alpha(0))+\left(\gamma_{0}\right)_{*} \alpha^{\prime}(0) .
$$

Since $\alpha(t)$ lies in the $\theta^{\infty}$-isotropic subset $V_{d}^{\infty}, \alpha^{\prime}(t)$ is in the kernel of $\theta^{\infty}$ and we arrive at the conclusion

$$
\theta^{\infty}\left(\gamma^{\prime}(\alpha(0))\right)=0
$$

But this quantity is nonzero since $\gamma^{\prime}$ is the Reeb vector field on $T^{\infty} X$.

5.2.1. Exact Lagrangians. A Lagrangian $i: L \hookrightarrow T^{*} X$ is said to be exact if the restriction $i^{*} \theta$ is an exact differential form.

Lemma 5.2.6. Let $L \subset T^{*} X$ be a Lagrangian, $\Sigma$ a compact Riemann surface with boundary $\partial \Sigma$, and $u:(\Sigma, \partial \Sigma) \rightarrow\left(T^{*} X, L\right)$ a differentiable map. Let $A(u)=\int_{\Sigma} u^{*} \omega$ denote its symplectic area. Then we have

(1) $A(u)$ depends only on the homotopy class in $L$ of $\left.u\right|_{\partial \Sigma}$.

(2) $A\left(u_{\epsilon}\right)=\epsilon A(u)$, where $u_{\epsilon}:(\Sigma, \partial \Sigma) \rightarrow\left(T^{*} X, \epsilon L\right)$ is the composition of $u$ with the dilation $(x, \xi) \mapsto(x, \epsilon \xi)$.

(3) If $L$ is exact, $u$ is constant.

(4) If $u$ is holomorphic, then Area $(u)=A(u)$.

Proof. For the first assertion, note that if $\gamma_{1}$ and $\gamma_{2}$ are homotopic loops in $L$ and $S \subset L$ satisfies $\partial S=\left[\gamma_{1}\right]-\left[\gamma_{2}\right]$, then $\oint_{\gamma_{1}} \theta-\oint_{\gamma_{2}} \theta=\int_{S} d \theta=\int_{S} \omega=0$, since $S \subset L$ is $\omega$-isotropic. To prove the second, for $p=(x, \xi) \in T^{*} X$, let $\epsilon p$ denote the point $(x, \epsilon \xi)$ and note that $\left.\theta\right|_{\epsilon p}(\epsilon v)=\left.\epsilon \theta\right|_{p} v$. The third claim follows from exactness: $\int_{\Sigma} u^{*} \omega=\oint_{\partial \Sigma} u^{*} \theta=\oint_{\partial \Sigma} u^{*}(d \psi)=0$. The fourth statement expresses the fact that when $J$ is a compatible almost complex structure, $J$-holomorphic maps are calibrations for $\omega$.

5.2.2. Standard Lagrangians. Let $Y \subset X$ be a submanifold. The conormal bundle $T_{Y}^{*} X \subset T^{*} X$ is homotopic to its zero section $Y$, and thus is an exact Lagrangian, since $\theta$ is identically zero on the zero section $X$.

Given a defining function $m: X \rightarrow \mathbb{R}_{\geq 0}$ for the boundary $\partial Y \subset X$, we define $f: X \backslash \partial Y \rightarrow \mathbb{R}$ by $f=\log m$ and define the standard Lagrangian $\left.L_{Y, f} \subset T^{*} X\right|_{Y} \subset$ $T^{*} X$ to be the fiberwise sum

$$
L_{Y, f}=T_{Y}^{*} X+\left.\Gamma_{d f}\right|_{Y},
$$

where $\left.\Gamma_{d f} \subset T^{*} X\right|_{X \backslash \partial Y}$ denotes the graph of $d f$ and the sum is taken fiberwise in $\left.T^{*} X\right|_{Y}$. By construction, $L_{Y, f}$ depends only on the restriction of $m$ to $Y$ : if two functions agree on $Y$, then over $Y$ their differentials differ by a section of the 
conormal $T_{Y}^{*} X$. For this reason, in the sequel we will often refer to $m$ and $f$ as functions on $Y$. Note that if $Y$ is an open submanifold, we could equivalently take $m$ to be a defining function for the complement $X \backslash Y$.

Lemma 5.2.7. $L_{Y, f}$ is canonically Hamiltonian isotopic to $T_{Y}^{*} X$. In particular, $L_{Y, f}$ is exact.

Proof. To avert potential confusion, it is worth pointing out that $T_{Y}^{*} X$ is not necessarily closed in $T^{*} X$, and we will move $L_{Y, f}$ through a family of Lagrangian submanifolds which are not necessarily closed. In the subset $\left.T^{*} X\right|_{Y} \subset T^{*} X$, consider the function $H=f \circ \pi$ and the associated Hamiltonian flow $\varphi_{H, t}$. One checks that $\varphi_{H, t}$ applied to the Lagrangian $L_{Y, f}$ takes it to its dilation $(1-t) \cdot L_{Y, f}$. In particular, when $t=1$, one arrives at the conormal Lagrangian $T_{Y}^{*} X$.

5.3. Brane structures. In order to define a Fukaya category of a symplectic manifold $M$, one needs a grading on the Lagrangian intersections and orientations of the relevant moduli spaces of holomorphic disks. (Alternatively, one could be satisfied with an ungraded version of the Fukaya category with characteristic 2 coefficients.) Topological obstructions to gradings come from the bicanonical bundle of $M$ and the Maslov class of Lagrangians. Orientation of the moduli spaces requires a relative pin structure on the Lagrangians, so that their second Stiefel-Whitney classes must be restrictions of a (common) class on $M \sqrt[3]{3}$ In this section, we show that all obstructions to these structures vanish for $M=T^{*} X$ and the Lagrangians of interest. In what follows, we always work with the canonical exact symplectic structure on $T^{*} X$, and the compatible almost complex structure induced by a Riemannian metric on $X$.

5.3.1. Bicanonical line. The almost complex structure on $T^{*} X$ allows us to define the holomorphic canonical bundle

$$
\kappa=\left(\wedge^{\operatorname{dim} X} T^{h o l} T^{*} X\right)^{-1} .
$$

In order to compare the squared phase of Lagrangian subspaces at different points of $T^{*} X$, we need a homotopy class of trivializations of the bicanonical bundle $\kappa^{\otimes 2}$.

Proposition 5.3.1. The bicanonical bundle $\kappa^{\otimes 2}$ of $T^{*} X$ is canonically trivial.

Proof. Since the zero section $X$ is a deformation retract of $T^{*} X$, it suffices to see that $\left.\kappa^{\otimes 2}\right|_{X}$ is canonically trivial. At the zero section, $T T^{*} X$ has a canonical splitting into vertical and horizontal spaces,

$$
\left.T T^{*} X\right|_{X}=\left.\left.T^{*} X \oplus T X\right|_{X} \cong T X \otimes \mathbb{C}\right|_{X},
$$

where we have identified the cotangent bundle with the tangent bundle using the metric, and identified the normal directions with the imaginary directions using the compatible almost complex structure. As a result,

$$
\left.T^{h o l}\left(T^{*} X\right)\right|_{X} \cong T X \otimes \mathbb{C},
$$

and we see

$$
\left.\kappa\right|_{X} \simeq \pi^{*}\left(\text { or }_{X}\right) \otimes \mathbb{C}
$$

\footnotetext{
${ }^{3}$ Some authors have proven orientability under more restrictive conditions. In [33], Lagrangians are assumed to be pin, while in 10 they are taken to be oriented and relatively spin. Our more general condition follows Wehrheim and Woodward's work in progress. We note that the Lagrangians of interest for us will be canonically pin when $X$ is pin, and canonically oriented and relatively spin when $X$ is oriented.
} 
where $\operatorname{or}_{X}$ is the orientation line bundle. Thus $\kappa$ is trivializable if and only if $X$ is orientable, and $\kappa^{\otimes 2}$ is canonically trivial for any $X$.

Remark 5.3.2. In general, trivializations of a complex line bundle over a space $X$ form a torsor over the group of maps $X \rightarrow \mathbb{C}^{*}$. Homotopy classes of trivializations form a torsor over the group $H^{1}(X, \mathbb{Z})$.

5.3.2. Grading. Let $\eta^{2}$ be the canonical trivialization of $\kappa^{\otimes 2}$, and let $\mathcal{L}_{a g_{T^{*} X}} \rightarrow$ $T^{*} X$ be the bundle of Lagrangian planes. We have the squared phase map

$$
\begin{aligned}
& \alpha: \mathcal{L} a g_{T^{*} X} \rightarrow U(1), \\
& \alpha(\mathcal{L})=\eta\left(\wedge^{\operatorname{dim} X} \mathcal{L}\right)^{2} /\left|\eta\left(\wedge^{\operatorname{dim} X} \mathcal{L}\right)\right|^{2} .
\end{aligned}
$$

For a Lagrangian $L \subset T^{*} X$ and a point $x \in L$, we obtain a map $\alpha: L \rightarrow U(1)$ by setting $\alpha(x)=\alpha\left(T_{x} L\right)$. The Maslov class $\mu(L) \in H^{1}(L)$ is the obstruction class

$$
\mu=\alpha^{*}(d t),
$$

where $d t$ is the standard one-form on $U(1)$. Thus $\alpha$ has a lift to a map $\widetilde{\alpha}: L \rightarrow \mathbb{R}$ if and only if $\mu=0$. Such a lift is called a grading of the Lagrangian.

Remark 5.3.3. Choices of gradings of a Lagrangian $L$ form a torsor over the group $H^{0}(L, \mathbb{Z})$.

Next we check that our standard Lagrangians have canonical gradings. Recall that to a submanifold $Y \subset X$ and a defining function $m: X \rightarrow \mathbb{R}_{\geq 0}$ for the boundary $\partial Y \subset X$, we have the standard Lagrangian

$$
L_{Y, f}=T_{Y}^{*} X+\Gamma_{d f} \subset T^{*} X,
$$

where $f: Y \rightarrow \mathbb{R}$ is given by $f=\log m$.

Proposition 5.3.4. The Maslov class $\mu\left(L_{Y, f}\right) \in H^{1}\left(L_{Y, f}\right)$ vanishes. In fact, there is a canonical grading of $L_{Y, f}$.

Proof. Since $L_{Y, f}$ is canonically Hamiltonian isotopic to $T_{Y}^{*} X$, it suffices to check the assertions for $T_{Y}^{*} X$. Furthermore, since $T_{Y}^{*} X$ is a vector bundle over $Y$, it suffices to check the assertions along $Y$. Let $\left\{e_{j}\right\}_{j=1}^{\operatorname{dim} X}$ be an orthonormal frame field for $X$ along $Y$ extending an orthonormal frame field $\left\{e_{j}\right\}_{j=1}^{\operatorname{dim} Y}$ for $Y$. Note that the zero section, and in particular the frame field $\left\{e_{j}\right\}_{j=1}^{\operatorname{dim} X}$, has a constant squared phase equal to the identity of $U(1)$. Thus we can equip it with the canonical constant grading given by 0 in $\mathbb{R}$. Then the frame field along $Y$ given by $\left\{e_{j}\right\}_{j=1}^{\operatorname{dim}} Y \cup$ $\left\{J e_{j}\right\}_{j=\operatorname{dim} Y+1}^{n}$ has a constant squared phase $(-1)^{\operatorname{codim}_{X} Y}$ in $U(1)$. Thus we can equip it with the canonical constant grading given by $-\left(\operatorname{codim}_{Y} X\right) \pi$ in $\mathbb{R}$.

We will see later that with the canonical grading on $L_{Y, f}$, the Fukaya morphism complex $\operatorname{hom}_{F u k\left(T^{*} X\right)}\left(L_{X}, L_{Y, f}\right)$ has cohomology equal to the cohomology $H^{*}(Y)$ with its usual grading. Here we have written $L_{X}$ for the zero section $T_{X}^{*} X$ with its canonical grading.

5.3.3. Relative pin structure. Recall first that the group $\operatorname{Pin}^{+}(n)$ is the double cover of $O(n)$ with center $\mathbb{Z} / 2 \mathbb{Z} \times \mathbb{Z} / 2 \mathbb{Z}[4$ A pin structure on a Riemannian manifold $L$ is a lift of the structure group of $T L$ to $\operatorname{Pin}^{+}(n)$. The obstruction to a pin

\footnotetext{
${ }^{4}$ There is another double cover $\operatorname{Pin}^{-}(n)$ with center $\mathbb{Z} / 4 \mathbb{Z}$.
} 
structure is the second Stiefel-Whitney class $w_{2}(L) \in H^{2}(L, \mathbb{Z} / 2 \mathbb{Z})$, and choices of pin structures form a torsor over the group $H^{1}(L, \mathbb{Z} / 2 \mathbb{Z})$.

A relative pin structure on a submanifold $L \hookrightarrow M$ with background class $[w] \in$ $H^{2}(M, \mathbb{Z} / 2 \mathbb{Z})$ can be defined as follows. Fix a Čech cocycle $w$ representing $[w]$, and let $\left.w\right|_{L}$ be its restriction to $L$. Then a pin structure on $L$ relative to $[w]$ can be defined to be an $\left.w\right|_{L}$-twisted pin structure on $T L$. Concretely, this can be represented by a $\operatorname{Pin}^{+}(n)$-valued Čech 1 -cochain on $L$ whose coboundary is $\left.w\right|_{L}$. Such structures are canonically independent of the choice of Čech representatives.

Remark 5.3.5. For a given background class $[w]$, choices of relative pin structures on $L$ form a torsor over the group $H^{1}(L, \mathbb{Z} / 2 \mathbb{Z})$.

We check that our standard Lagrangians have canonical relative pin structures with respect to a canonical universal background class. Recall that to a submanifold $Y \subset X$, and a defining function $m: X \rightarrow \mathbb{R}_{>0}$ for the boundary $\partial Y \subset X$, we have the standard Lagrangian

$$
L_{Y, f}=T_{Y}^{*} X+\Gamma_{d f} \subset T^{*} X,
$$

where $f: Y \rightarrow \mathbb{R}$ is given by $f=\log m$.

Proposition 5.3.6. The second Stiefel-Whitney class $w_{2}\left(L_{Y, f}\right) \in H^{2}\left(L_{Y, f}, \mathbb{Z} / 2 \mathbb{Z}\right)$ is the restriction of $\pi^{*}\left(w_{2}(X)\right)$. In fact, there is a canonical relative pin structure on $L_{Y, f}$ with background class $\pi^{*}\left(w_{2}(X)\right)$.

Proof. Since there is a canonical homotopy class of isotopies between $L_{Y, f} \hookrightarrow T^{*} X$ and $T_{Y}^{*} X \hookrightarrow T^{*} X$, it suffices to check the assertion for the latter. The metric provides a canonical isomorphism between the restriction $\left.T T_{Y}^{*} X\right|_{Y}$ and the restriction $\left.T X\right|_{Y}$. By functoriality, we have the desired relative pin structure.

5.3.4. Definition of brane structures. Finally, we have the definition of a brane structure on a Lagrangian.

Definition 5.3.7 (33]). A brane structure $b$ on a Lagrangian submanifold $L \subset$ $T^{*} X$ is a pair $b=(\widetilde{\alpha}, P)$, where $\widetilde{\alpha}: L \rightarrow \mathbb{R}$ is a lift of the squared phase map and $P$ is a relative pin structure on $L$.

We have seen in the above discussion that our standard Lagrangians come equipped with canonical brane structures. We refer to a standard Lagrangian equipped with its canonical brane structure as a standard brane.

5.4. Definition of Fukaya category. In this section, we define the Fukaya $A_{\infty}$ category $F u k\left(T^{*} X\right)$. General foundations are taken largely from 33, and we restrict the discussion here to issues arising from noncompact Lagrangians. We assume $X$ is a compact, Riemannian, real analytic manifold, and equip $T^{*} X$ with its canonical exact symplectic structure. Throughout what follows, we fix an analyticgeometric category $\mathcal{C}$ and assume all subsets are $\mathcal{C}$-subsets unless otherwise noted.

5.4.1. Objects. Fix once and for all the canonical trivialization $\eta^{2}$ of the bicanonical bundle $\kappa^{2}$ and the background relative pin class $\pi^{*}\left(w_{2}(X)\right)$. All brane structures will be with reference to these fixed structures. The following should be considered a preliminary definition until we discuss perturbations. 
Definition 5.4.1. Objects of $F u k\left(T^{*} X\right)$ are quadruples $(L, \mathcal{E}, b, \Phi)$, where $L \subset$ $T^{*} X$ is an exact Lagrangian submanifold such that $\bar{L} \subset \bar{T}^{*} X$ is a $\mathcal{C}$-subset, $\mathcal{E} \rightarrow L$ is a vector bundle with flat connection, $b=(\widetilde{\alpha}, P)$ is a brane structure on $L$, and $\Phi$ is a collection of perturbations to be explained below.

When circumstances are clear, we often refer to an object of $F u k\left(T^{*} X\right)$ by its corresponding support Lagrangian. Given a submanifold $Y \subset X$ equipped with a local system $\mathcal{L}_{Y}$, we refer to a standard Lagrangian $L_{Y, f}$ equipped with the flat bundle $\mathcal{E}=\pi^{*}\left(\mathcal{L}_{Y} \otimes o r_{X} \otimes o r_{Y}^{-1}\right)$, and its canonical brane structure $b$ as a standard object.

We have defined the objects from the point of view of the compactified cotangent bundle $\bar{T}^{*} X$ in order to give a cleaner definition of the Lagrangians of interest. Requiring $\bar{L}$ to be a $\mathcal{C}$-subset of $\bar{T}^{*} X$ excludes various types of behavior near infinity $T^{\infty} X$. For example, with our definition, we cannot have infinitely many intersection points (as might occur for a helix on $T^{*} S^{1}$ ). Although we rule this out from the beginning, certain theories of the Fukaya category on $T^{*} X$ allow such behavior. Note that we use $\bar{T}^{*} X$ as a topological compactification, but not as a symplectic compactification. From the point of view of constructing moduli spaces (see below), our Lagrangians are noncompact.

5.4.2. Morphisms. To define the morphisms between two objects, we need to choose Hamiltonian isotopies to move their underlying Lagrangians so that they do not intersect at infinity, and have transverse intersections in finite space. As usual, the intersections will depend on the choice of isotopies, but in a homotopically manageable way. First, we explain a broad class of isotopies which provide a consistent topological form for the intersections of our Lagrangians. In the next section, we assume the existence of a more restricted class of isotopies which guarantee that we may use moduli spaces of holomorphic disks to define composition maps.

Recall that a Hamiltonian function $H: T^{*} X \rightarrow \mathbb{R}$ is said to be controlled if there is a compact set $K \subset T^{*} X$ such that outside of $K$ we have $H(x, \xi)=|\xi|$. The corresponding Hamiltonian isotopy $\varphi_{H, t}: T^{*} X \rightarrow T^{*} X$ equals the normalized geodesic flow $\gamma_{t}$ outside of $K$. By Lemma 5.2.5 for Lagrangians $L_{0}, L_{1} \subset T^{*} X$, we may choose controlled Hamiltonian functions $H_{0}, H_{1}$ and a fringed set $R \subset \mathbb{R}^{2}$ such that for $\left(\delta_{0}, \delta_{1}\right) \in R$, there is $r>0$ such that

$$
\varphi_{H_{0}, \delta_{0}}\left(\bar{L}_{0}\right) \cap \varphi_{H_{1}, \delta_{1}}\left(\bar{L}_{1}\right) \subset T^{*} X_{|\xi|<r},
$$

and the intersection is transverse. Suppose that we consider objects of $F u k\left(T^{*} X\right)$ to come equipped with such data and that the brane structures and bundles are transported via the perturbations. Then we may make the following definition.

Definition 5.4.2. For objects $L_{0}, L_{1}$ of $F u k\left(T^{*} X\right)$, the space of morphisms is defined to be

$$
\operatorname{hom}_{F u k\left(T^{*} X\right)}\left(L_{0}, L_{1}\right)=\bigoplus_{p \in \varphi_{H_{0}, \delta_{0}}\left(L_{0}\right) \cap \varphi_{H_{1}, \delta_{1}}\left(L_{1}\right)} \operatorname{Hom}\left(\left.\mathcal{E}_{0}\right|_{p},\left.\mathcal{E}_{1}\right|_{p}\right)[-\operatorname{deg}(p)] .
$$

The integer $\operatorname{deg}(p)$ denotes the Maslov grading, or index, of the linear Lagrangian subspaces at the intersection; see Sections 11e-11g of [33].

The differential on the complex of morphisms will be defined in Section 5.4.4 below along with all of the higher composition maps. 
5.4.3. Holomorphic disks. The composition maps of the Fukaya $A_{\infty}$-category are defined by counting points (with orientations) in appropriate moduli spaces of holomorphic maps with respect to a compatible almost complex structure. To ensure that the moduli spaces are well behaved, one must consider further perturbations, as described by Seidel [33. One must choose Floer perturbation data consisting of a time-dependent Hamiltonian function and almost complex structure deformation. One must also choose perturbation data on the Riemann surfaces to be mapped.

At the same time, we must check that the moduli spaces are compact. This is delicate due to the fact that our Lagrangians are not necessarily compact. For the case of closed Riemann surfaces and no Lagrangians, if we consider surfaces intersecting a fixed compact set and with area less than a uniform upper bound, then tameness of the target in the sense of [35, 2] ensures compact moduli spaces. It is a standard fact that $T^{*} X$, with its canonical symplectic form and the Sasaki almost complex structure $J_{\text {Sas }}$ associated to a Riemannian metric on $X$, is tame. To deal with the boundary case with standard Lagrangians as boundary, it will be useful to consider the conical almost complex structure $J_{c o n}$ introduced in Section 5.1.3. Recall that near infinity, the conical metric $g_{c o n}$ presents $T^{*} X$ as a cone over the unit (co-)sphere bundle $S^{*} X$. It is straightforward to calculate explicitly that such a metric has sectional curvature bounded from above and injectivity radius bounded away from zero. Thus $T^{*} X$ equipped with the conical almost complex structure $J_{\text {con }}$ is tame.

For the Lagrangian boundary case, one typically imposes additional conditions on the Lagrangian submanifolds themselves. The fact that our Lagrangians are exact means that none of the complications from the bubbling of spheres will be present, but compactness still must be ensured. To achieve compact moduli spaces, there are many possible strategies involving assumptions adapted to different situations. The situation for standard branes is robust: one can successfully apply a diverse host of techniques including convexity statements, dilation arguments, and energy bounds. Thus in the context of this paper, our choice of a specific uniform definition of $F u k\left(T^{*} X\right)$ is largely aesthetic. As with our use of Lagrangians lying in some analytic-geometric category, we have decided upon the approach described below since it is particularly easy to work with in applications to mirror symmetry and representation theory. We will apply the sufficient tameness conditions for compact moduli spaces derived in [35, 2]. They require that (1) there exists $\rho_{L}>0$ such that for every $x \in L$, the set of points $y \in L$ with $d(x, y) \leq \rho_{L}$ is contractible, and that (2) there exists $C_{L}$ giving a two-point condition $d_{L}(x, y) \leq C_{L} d(x, y)$ whenever $x, y \in L$ with $d(x, y)<\rho_{L}$.

As the following example shows, standard Lagrangians do not necessarily satisfy the above conditions with respect to the Sasaki metric $g_{\text {Sas }}$.

Example 5.4.3. Consider any smooth curve $C \subset \mathbb{R}^{2}$ and its conormal bundle $T_{C}^{*} \mathbb{R}^{2} \subset T^{*} \mathbb{R}^{2}$. If $C$ has an inflection point, then $T_{C}^{*} \mathbb{R}^{2}$ is not tame with respect to the Sasaki metric $g_{\text {Sas }}$. Note that having an inflection point is a generic circumstance.

The reason for the above phenomenon is that the Sasaki metric $g_{\text {Sas }}$ is very asymmetric: vertical directions along co-sphere bundles grow with the radius, while horizontal directions remain a fixed length. We have introduced the conical metric $g_{c o n}$ to remedy the situation. It is straightforward to check that for any compact submanifold $Y \subset X$, the conormal bundle $T_{Y}^{*} X \subset T^{*} X$ is tame with respect to 
the conical metric $g_{\text {con }}$ (near infinity, the conormal is nothing more than a smooth subcone of the ambient cone). While this fact is crucial, it is not the end of the story. As the following example shows, standard Lagrangians associated to submanifolds with singular boundaries are not necessarily tame with respect to $g_{c o n}$.

Example 5.4.4. Fix a point $p \in \mathbb{R}^{2}$ and a smooth closed curve $c:[0, \infty) \rightarrow \mathbb{R}^{2}$ with $c(0)=p$. Consider the complement $U=\mathbb{R}^{2} \backslash c$ and a standard Lagrangian $L_{U} \subset T^{*} \mathbb{R}^{2}$ associated to $U$. If $c$ has nonvanishing curvature in a neighborhood of $p$, then $L_{U}$ will not be tame with respect to the conical metric $g_{c o n}$. Note that nonvanishing curvature is a generic circumstance.

Now to ensure that we have compact moduli spaces, we will give ourselves some added flexibility and assume that our Lagrangains come equipped with perturbations $\Phi$ making them tame near infinity with respect to the conical metric $g_{\text {con }}$. For our intended applications, this assumption is easily verified and poses no further restrictions. (Furthermore, if one is willing to work with immersed but not necessarily embedded Lagrangians, one could expect that there is no obstruction to finding such perturbations. Since the foundations of the Fukaya category of immersed Lagrangians are still not available in the literature, we will not pursue this direction and insist for now that the perturbations exist.)

To make this precise, for a brane $L$ to define an object of $F u k\left(T^{*} X\right)$, we require the existence of the following further perturbation data. First, by a perturbation of $L$, we mean a one-parameter family of branes $L_{t} \subset T^{*} X \times \mathbb{R}$ such that $L_{0}=L$ and for $|\xi|>r>0$ sufficiently large, the product map $L_{t} \rightarrow \mathbb{R} \times(r, \infty)$ given by the parameter $t$ and the length $|\xi|$ is a proper submersion. (This guarantees that the family of branes has constant topology near infinity.) Now fix a defining function $n: \bar{T}^{*} X \rightarrow \mathbb{R}_{\geq 0}$ for the closure $\bar{L} \subset \bar{T}^{*} X$, and for $\varepsilon>0$, let $N_{\varepsilon}(\bar{L}) \subset \bar{T}^{*} X$ be the open neighborhood $\bar{T}^{*} X_{n<\varepsilon}$. Given a brane $L$, we require the existence of a smooth perturbation $L_{t}$ such that for all $\varepsilon>0$, the brane $L_{\varepsilon}$ is tame with respect to the conical metric $g_{\text {con }}$ and lies in $N_{\varepsilon}(L)$. It is worth pointing out that we do not insist that the family $L_{t}$ is uniformly tame with respect to $t$. (In fact, if the original brane $L$ is not tame, then of course it is impossible to find a uniformly tame perturbation.) Similarly, we do not insist that the family $L_{t}$ extends to infinity. Neither circumstance causes any harm.

Lemma 5.4.5. Standard Lagrangians admit perturbations to Lagrangians tame with respect to the conical metric $g_{\text {con }}$.

Proof. We will provide a concrete perturbation for standard branes. The interested reader will note that the underlying construction is quite general and could be applied to many branes. We will move our brane to a new brane $L \subset T^{*} X$ such that in a neighborhood of infinity, its closure $\bar{L} \subset \bar{T}^{*} X$ is diffeomorphic to the product $L^{\infty} \times(r, \infty]$ of the boundary $L^{\infty} \subset T^{\infty} X$ with an interval. Moreover, near infinity the new brane $L$ will be uniformly close to the cone over its boundary $L^{\infty}$. Since cones over compact submanifolds are tame with respect to the conical metric $g_{c o n}$, this will immediately imply that $L$ is also tame. In fact, though it is unnecessary, we could go one step further and move $L$ so that near infinity it is equal to the cone over its boundary $L^{\infty}$.

With these general remarks in mind, let us turn to the case at hand of standard branes. Fix a submanifold $Y \subset X$ and a defining function $m: X \rightarrow \mathbb{R}_{\geq 0}$ for the boundary $\partial Y \subset X$, and consider the standard Lagrangian $L_{Y, f}=\Gamma_{d f} \mid Y^{+}+T_{Y}^{*} X \subset$ 
$\left.T^{*} X\right|_{Y} \subset T^{*} X$ where as usual $f=\log m$. Our first step will be to move $L_{Y, f}$ to a standard Lagrangian associated to a submanifold with smooth boundary. For small $\eta>0$, choose an increasing function $b_{\eta}: \mathbb{R} \rightarrow \mathbb{R}$ satisfying $b_{\eta}(s)=s$ for $s \geq \eta$ and $b_{\eta}(s)=0$ for $s \leq 0$. Consider the composition $m_{\eta}=b \circ m$ and the submanifold $Y_{\eta}=Y_{m_{\eta}>0} \subset Y$. Then for all sufficiently small $\eta>0$, the boundary $\partial Y_{\eta} \subset X$ is a smooth submanifold. Our first step is to perturb $L_{Y, f}$ to the standard Lagrangian $L_{Y_{\eta}, f_{\eta}}=\Gamma_{d f_{\eta}}\left|Y_{\eta}+T_{Y_{\eta}}^{*} X \subset T^{*} X\right|_{Y_{\eta}} \subset T^{*} X$ where we have set $f_{\eta}=\log m_{\eta}$.

Let us assess what we have accomplished so far. If $Y$ is open, then we claim that $L_{Y_{\eta}, f_{\eta}}$ is tame. To see this, observe that $\partial Y_{\eta}$ is a smooth hypersurface with normal coordinate $m$. Thus one can check directly in local coordinates that the conormal bundle $T_{\partial Y_{\eta}}^{*} X$ uniformly approximates $L_{Y_{\eta}, f_{\eta}}$ near infinity. If $Y$ is not open, then we must add a second step to our perturbation. In this case, observe that $\bar{Y}_{\eta}$ is a smooth submanifold with boundary $\partial Y_{\eta}$ with normal coordinate $m$. Thus one can check directly in local coordinates that in a neighborhood of infinity, the closure $\bar{L}_{Y_{\eta}, f_{\eta}} \subset \bar{T}^{*} X$ is homeomorphic to the product $L_{Y_{\eta}, f_{\eta}}^{\infty} \times(r, \infty]$ of the boundary $L_{Y_{\eta}, f_{\eta}}^{\infty} \subset T^{\infty} X$ with an interval. Moreover, the boundary $L_{Y_{\eta}, f_{\eta}}^{\infty}$ is a piecewise smooth Legendrian (if $Y$ is closed, then it is in fact smooth; if $Y$ is not closed, it is a union of two submanifolds glued along their boundaries). Therefore we can perturb $L_{Y_{\eta}, f_{\eta}}^{\infty}$ to a smooth nearby Legendrian $\mathcal{L}^{\infty}$, and hence perturb $L_{Y_{\eta}, f_{\eta}}$ to a nearby Lagrangian which near infinity is uniformly approximated by the cone over $\mathcal{L}^{\infty}$

Before continuing, let us make a couple of remarks. First, a comment about the application of the above perturbations in the next section: given a brane $L$ admitting such a perturbation, it follows that its image under a controlled Hamiltonian isotopy does so as well. Namely, we can simply conjugate the perturbation by the isotopy since normalized geodesic flow is an isometry of the conical metric. It is in this form that we will use the discussion of this section. Second, although it is not necessary for the aim of this paper, it is worth pointing out that one could allow the flexibility of any compatible almost complex structure as long as some $C^{0}$-bounds were obtained. What we have described is a simple concrete framework to deal with standard branes and objects which arise in our intended applications.

5.4.4. Composition maps. Now we are ready to define the composition maps of the Fukaya $A_{\infty}$-category $\mathrm{Fuk}\left(T^{*} X\right)$.

Let $L_{0}, \ldots, L_{d}$ be a finite collection of objects of $F u k\left(T^{*} X\right)$. By Lemma 5.2.5, we may choose controlled Hamiltonian functions $H_{i}: T^{*} X \rightarrow \mathbb{R}$, for $i=0, \ldots, d$, and a fringed set $R \subset \mathbb{R}^{d+1}$ such that for $\left(\delta_{0}, \ldots, \delta_{d}\right) \in R$, there is $r>0$ such that

$$
\varphi_{H_{i}, \delta_{i}}\left(\bar{L}_{i}\right) \cap \varphi_{H_{j}, \delta_{j}}\left(\bar{L}_{j}\right) \subset T^{*} X_{|\xi|<r} \text {, for } i \neq j,
$$

and the intersections are transverse. Then as discussed in the previous section, by assumption, we may apply further small perturbations so that the resulting branes are tame with respect to the conical almost complex structure.

We consider objects of $F u k\left(T^{*} X\right)$ to come equipped with such data. Then by the results of [35], the moduli spaces of holomorphic maps from a disk with a fixed number of marked boundary points taken to intersection points and boundary arcs taken to individual branes is compact. In fact, by [35, we have an explicit diameter bound on the image of any such holomorphic map. From this, one can verify that our perturbation framework can be handled by standard techniques: 
the fringed set at infinity adds only a contractible space of data to the usual Floer theory perturbations with compact support, and one checks that the remaining noncompact perturbations lead to canonical isomorphisms. (See 28, for a discussion of setting up the relevant continuation maps.) In summary, the usual methods of organizing the perturbation data to obtain a well-defined $A_{\infty}$-category apply (we refer the reader to the careful account in 33, Chapters 9 and 12). For example, for a compactly supported time-dependent Hamiltonian $H_{t}$, moduli spaces with moving boundary conditions provide isomorphisms between the identity functor and the family of functors $\varphi_{H_{t}, T}$. (See 28, for a discussion of which noncompactly supported Hamiltonian isotopies provide isomorphisms.)

With the preceding in hand, we define the $A_{\infty}$-composition maps of $F u k\left(T^{*} X\right)$ as usual by their structure constants: they count the signed number of holomorphic maps from a disk with $d+1$ marked boundary points with the appropriate boundary conditions.

Definition 5.4.6. For $L_{0}, \ldots, L_{d}$ objects of $F u k\left(T^{*} X\right)$, one defines

$$
\begin{aligned}
m_{F u k\left(T^{*} X\right)}^{d}: & \operatorname{hom}_{F u k\left(T^{*} X\right)}\left(L_{0}, L_{1}\right) \otimes \cdots \otimes \operatorname{hom}_{F u k\left(T^{*} X\right)}\left(L_{d-1}, L_{d}\right) \\
& \rightarrow \operatorname{hom}_{F u k\left(T^{*} X\right)}\left(L_{0}, L_{d}\right)
\end{aligned}
$$

as follows. Consider elements $p_{i} \in \operatorname{hom}_{F u k\left(T^{*} X\right)}\left(L_{i}, L_{i+1}\right)$, for $i=0, \ldots, d-1$, and $p_{d} \in \operatorname{hom}_{F u k\left(T^{*} X\right)}\left(L_{0}, L_{d}\right)$. Then the coefficient of $p_{d}$ in $m_{F u k\left(T^{*} X\right)}^{d}\left(p_{0}, \ldots, p_{d-1}\right)$ is the signed sum over holomorphic maps from a disk with $d+1$ counterclockwise cyclically ordered marked points mapping to $p_{i}$ and corresponding boundary arcs mapping to $L_{i+1}$. Each map contributes according to the holonomy of its boundary, where adjacent components $L_{i}$ and $L_{i+1}$ are glued with $p_{i}$.

By Lemma 5.2.6 there is no bubbling of spheres in the Fukaya category of an exact symplectic manifold. As a result, the Novikov coefficient rings employed to account for all possible areas of maps are unnecessary, and we content ourselves with simply counting the maps, with no weighting by areas. This simplifies the isomorphism of different perturbation data, since we need not keep track of the changes in the areas of disks as the intersection points move.

\section{EMBEDding OF STANDARD OBJECTS}

In this section we will construct an embedding of the Morse $A_{\infty}$-category $\operatorname{Mor}(X)$ into the Fukaya $A_{\infty}$-category $F u k\left(T^{*} X\right)$. The embedding relies on rather delicate and detailed perturbations of a collection of standard Lagrangians. After the necessary preparations, we will be able to understand the moduli spaces of holomorphic polygons bounding our perturbed Lagrangians in terms of Morse theory via the theorem of Fukaya and Oh $[9]$.

6.1. Preliminaries. Here we recall some of our conventions and notation concerning the geometry of the cotangent bundle $\pi: T^{*} X \rightarrow X$. Throughout what follows, we identify $X$ with the zero section in $T^{*} X$. We fix a Riemannian metric on $X$, and write $d_{X}(x, y)$ for the distance between points $x, y \in X$.

Under the metric identification $T\left(T^{*} X\right) \simeq T(T X)$, the canonical one-form $\theta$ on $T^{*} X$ corresponds to the geodesic vector field $v_{\theta}$. On the complement of the zero section $T^{*} X \backslash X$, we have the normalized geodesic vector field $\hat{v}_{\theta}=v_{\theta} /\left|v_{\theta}\right|$. It is the Hamiltonian vector field for the length function $H: T^{*} X \backslash X \rightarrow \mathbb{R}$ given by 
$H(x, \xi)=|\xi|$. We write $\gamma_{t}: T^{*} X \backslash X \rightarrow T^{*} X \backslash X$ for the normalized geodesic flow for time $t$ associated to $\hat{v}_{\theta}$. By definition, if we identify a covector $(x, \xi) \in T^{*} X$ with a vector $(x, v) \in T X$, then we have the identity

$$
\gamma_{t}(x, v)=\exp _{x, t}(\hat{v})_{*}(v),
$$

where $\hat{v}=v /|v|$, the map $\exp _{x, t}: T_{x} X \rightarrow X$ denotes the exponential flow from the point $x$ for time $t$, and the asterisk subscript indicates the derivative (push-forward). Note that for $t$ sufficiently small - for example, less than half the injectivity radius of $X$ - we have $d_{X}\left(\pi(x, \xi), \pi\left(\gamma_{t}(x, \xi)\right)=t\right.$.

Given a stratification $\mathcal{S}=\left\{S_{\alpha}\right\}$ of $X$, we define the associated conical Lagrangian $\Lambda_{\mathcal{S}} \subset T^{*} X$ to be the union of conormals

$$
\Lambda_{\mathcal{S}}=\bigcup_{\alpha} T_{S_{\alpha}}^{*} X .
$$

Given a second stratification $\mathcal{S}^{\prime}$ refining $\mathcal{S}$, note the corresponding inclusion $\Lambda_{\mathcal{S}} \subset$ $\Lambda_{\mathcal{S}^{\prime}}$

Some usual notation: given a space $Y$, a function $g: Y \rightarrow \mathbb{R}$, and $r \in \mathbb{R}$, we write $Y_{g=r}$ for the subset $\{y \in Y \mid g(y)=r\}$, and similarly for inequalities.

6.2. Variable dilation. We will need to dilate a standard Lagrangian so that it is as close as we would like to its associated conical Lagrangian. To achieve this in a controlled fashion, we must consider two regions: (1) a neighborhood of infinity where the Lagrangian is already close to its associated conical Lagrangian, and (2) a compact region where dilation of the standard Lagrangian is a Hamiltonian isotopy.

Let $U \subset X$ be an open submanifold, with closure $\bar{U} \subset X$ and boundary $\partial U=$ $\bar{U} \backslash U$. Fix a defining function $m: X \rightarrow \mathbb{R}_{>0}$ for the closed subset $X \backslash U$, and let $f: U \rightarrow \mathbb{R}$ be the function $f=\log m$. Let $L \subset T^{*} X$ be the standard Lagrangian given by the differential of $f$.

Lemma 6.2.1. For any $\ell>0$, there is $\eta>0$ such that $L_{m<\eta} \subset L_{|\xi|>\ell}$. For any $\eta>0$, there is $\ell>0$ such that $L_{m>\eta} \subset L_{|\xi|<\ell}$.

Proof. Immediate from the definitions.

Choose a stratification of $X$ which refines the boundary $\partial U \subset X$, and let $\Lambda \subset$ $T^{*} X$ denote the associated conical Lagrangian. Choose a defining function $n$ : $\bar{T}^{*} X \rightarrow \mathbb{R}_{\geq 0}$ for the closure $\bar{\Lambda}$, and for any $\varepsilon \geq 0$, let $N_{\varepsilon}(\Lambda) \subset T^{*} X$ denote the open neighborhood $T^{*} X_{n<\varepsilon}$ of $\Lambda$.

Lemma 6.2.2. For any $\varepsilon>0$, there is $\ell>0$ such that

$$
L_{|\xi| \geq \ell} \subset N_{\varepsilon}(\Lambda)
$$

Proof. Recall that for a subset $Y \subset T^{*} X$, we write $Y^{\infty}$ for the intersection of the closure $\bar{Y} \subset \bar{T}^{*} X$ with the divisor at infinity $T^{\infty} X \subset \bar{T}^{*} X$. The assertion follows immediately from the inclusion $L^{\infty} \subset \Lambda^{\infty}$.

Fix positive numbers $a<b \in(0, \infty)$, and choose an increasing function $d_{a, b}$ : $\mathbb{R} \rightarrow \mathbb{R}$ satisfying the following:

$$
d_{a, b}(r)=\left\{\begin{array}{cl}
r, & \text { for } r \geq \log b \\
\log \sqrt{a b}, & \text { for } r \leq \log a .
\end{array}\right.
$$


In order to dilate the Lagrangian $L$, we consider the Hamiltonian flow $\varphi_{D_{a, b}, t}$ : $T^{*} X \rightarrow T^{*} X$ generated by the function $D_{a, b}: T^{*} X \rightarrow \mathbb{R}$ defined by

$$
D_{a, b}(x, \xi)=\left\{\begin{array}{cl}
-d_{a, b}(\log m(x)), & \text { for }(x, \xi) \text { with } m(x) \neq 0 \\
-\log \sqrt{a b}, & \text { otherwise. }
\end{array}\right.
$$

The motion of the Lagrangian $L$ under the flow $\varphi_{D_{a, b}, t}$ is given by the variable dilation

$$
\varphi_{D_{a, b}, t}(L)=\left(1-t d_{a, b}^{\prime}(\log m(x))\right) \cdot L .
$$

In particular, the Lagrangian $\varphi_{D_{a, b}, t}(L)$ continues to be a graph over $U$, and coincides with $L$ over $m \leq a$ and with $(1-t) \cdot L$ over $m \geq b$.

Lemma 6.2.3. For any $\varepsilon>0$, there is $b>0$ and $\delta>0$ such that for all $a^{\prime}, b^{\prime} \in$ $(0, b), a^{\prime}<b^{\prime}$, and $\delta^{\prime}>\delta$, we have

$$
\varphi_{D_{a^{\prime}, b^{\prime}}, \delta^{\prime}}(L) \subset N_{\varepsilon}(\Lambda) .
$$

Proof. By the previous lemmas, we may choose $b>0$ so that

$$
L_{m \leq b} \subset N_{\varepsilon}(\Lambda)
$$

Since $\Lambda$ is conical, $\varphi_{D_{a, b}, t}$ preserves $N_{\varepsilon}(\Lambda)$. Thus we need only choose $\delta>0$ so that

$$
\varphi_{D_{a, b}, \delta}\left(L_{m \geq b}\right) \subset N_{\varepsilon}(\Lambda) .
$$

But $L_{m \geq b}$ is a compact set, and $\Lambda$ contains the zero section of $T^{*} X$.

6.3. Separation. Here we discuss how to perturb a standard Lagrangian near infinity. Namely, we show that near infinity we may separate it from a conical Lagrangian without disturbing its structure elsewhere.

Let $U \subset X$ be an open submanifold with closure $\bar{U} \subset X$ and boundary $\partial U=$ $\bar{U} \backslash U$. Fix a defining function $m: X \rightarrow \mathbb{R}_{\geq 0}$ for $X \backslash U$, and let $f: U \rightarrow \mathbb{R}$ be the function $f=\log m$. Let $L \subset T^{*} X$ be the standard Lagrangian given by the graph of $d f$.

Let $\mathcal{S}=\left\{S_{\alpha}\right\}$ be any stratification of $X$, and let $\Lambda_{\mathcal{S}} \subset T^{*} X$ be the corresponding conical Lagrangian

$$
\Lambda_{\mathcal{S}}=\bigcup_{\alpha} T_{S_{\alpha}}^{*} X
$$

Note that we do not assume that $\mathcal{S}$ has any relation to $U$ or its boundary $\partial U$.

We say that $x \in X$ is a $\Lambda_{\mathcal{S}}$-critical point of $m$ if we have $d m(x) \in \Lambda_{\mathcal{S}}$. Note that for $x \in U$ this is the same as $d f(x) \in \Lambda_{\mathcal{S}}$ since $d f=d m / m$ and $\Lambda_{\mathcal{S}}$ is conical. We say that $r \in \mathbb{R}$ is a $\Lambda_{\mathcal{S}}$-critical value of $m$ if there is a $\Lambda_{\mathcal{S}}$-critical point $x \in X$ such that $r=m(x)$.

Lemma 6.3.1. There is $\eta>0$ so that there are no $\Lambda_{\mathcal{S}}$-critical values of $m: X \rightarrow$ $\mathbb{R}_{\geq 0}$ in the interval $(0, \eta]$.

Proof. The $\Lambda_{\mathcal{S}}$-critical values of $m$ form a discrete subset of $\mathbb{R}$.

The following strengthening of Lemma 5.2.5 will simplify our perturbations, as we can choose the parameter $\eta>0$ to be independent of sufficiently small $\delta>0$.

Lemma 6.3.2. There exist $\eta>0$ and $\delta>0$ such that for all $\delta^{\prime} \in(0, \delta]$, the normalized geodesic flow satisfies

$$
\gamma_{\delta^{\prime}}\left(\bar{L}_{m \leq \eta}\right) \cap \bar{\Lambda}_{\mathcal{S}}=\emptyset .
$$


Proof. We prove the assertion by contradiction. So suppose it were false.

First, recall that for all sufficiently small $\delta>0$, we have seen in Lemma 5.2 .5 that

$$
\gamma_{\delta}\left(L^{\infty}\right) \cap \Lambda_{\mathcal{S}}^{\infty}=\emptyset .
$$

Thus by the previous lemma, if the assertion were false, then by the curve selection lemma there exists a $\delta>0$ and a subanalytic curve

$$
\ell(t)=(x(t), \xi(t)):[0, \delta) \rightarrow L
$$

such that $m(x(t)) \rightarrow 0$ as $t \rightarrow 0$, and (after a possible reparametrization) we have

$$
\gamma_{t}(\ell(t)) \in T_{S_{\alpha}}^{*} X \text {, for all } t \in(0, \delta),
$$

for some fixed stratum $S_{\alpha}$ (we may fix $\alpha$ since there are finitely many strata).

Let $\kappa(t)=(y(t), \zeta(t)) \in \Lambda_{\mathcal{S}}$ denote the image curve $\gamma_{t}(\ell(t))$. Again by definition, if we identify the covector $(x(t), \xi(t)) \in T^{*} X$ with a vector $(x(t), v(t)) \in T X$, then we have

$$
\zeta(t)(w)=\left\langle\exp _{x(t), t} \hat{v}(t), w\right\rangle=0, \text { for all } t \in(0, \delta) \text {, and } w \in T_{y(t)} S_{\alpha} .
$$

Let $x^{\prime}(t)$ denote the tangent vector to the curve $x(t)$. Since $m(x(t)) \rightarrow 0^{+}$as $t \rightarrow 0^{+}$, we have the inequality

$\xi(t)\left(x^{\prime}(t)\right)=\left\langle v(t), x^{\prime}(t)\right\rangle=\left\langle\nabla f(x(t)), x^{\prime}(t)\right\rangle=\frac{d}{d t} f(x(t))=\frac{1}{m(x(t))} \frac{d}{d t} m(x(t))>0$

for $t$ sufficiently small.

On the other hand, observe that $d_{X}(x(t), y(t))=t$, so that

$$
\frac{d}{d t} d_{X}(x(t), y(t))=1 \text {. }
$$

But in general, consider $x, y \in X$ connected by a geodesic with tangent vector $v_{x}$ at $x$ and $v_{y}$ at $y$. Then for any curves $x(t), y(t)$ in $X$, with $x\left(t_{0}\right)=x, y\left(t_{0}\right)=y$, we have

$$
\left.\frac{d}{d t} d_{X}(x(t), y(t))\right|_{t=t_{0}}=\left\langle y^{\prime}\left(t_{0}\right), v_{y}\right\rangle-\left\langle x^{\prime}\left(t_{0}\right), v_{x}\right\rangle .
$$

In the case at hand, $v_{x}=\hat{v}(t)$ and $v_{y}$ is its image under $\exp _{x(t), t}$. But we have seen that $\left\langle y^{\prime}\left(t_{0}\right), v_{q}\right\rangle=0$, since $y^{\prime}\left(t_{0}\right) \in T_{S_{\alpha}}^{*} X$, and also that $\left\langle x^{\prime}\left(t_{0}\right), v_{x}\right\rangle>0$. Thus we have

$$
\left.\frac{d}{d t} d_{X}(x(t), y(t))\right|_{t=t_{0}}<0
$$

and we have arrived at a contradiction.

Fix positive numbers $k<\ell \in(0, \infty)$, and choose an increasing function $g_{k, \ell}$ : $\mathbb{R} \rightarrow \mathbb{R}$ satisfying the following

$$
g_{k, \ell}(r)=\left\{\begin{array}{cl}
r, & \text { for } r \geq \ell, \\
(k+\ell) / 2, & \text { for } r \leq k .
\end{array}\right.
$$

Consider the Hamiltonian flow $\varphi_{G_{k, \ell}, t}: T^{*} X \rightarrow T^{*} X$ generated by the function $G_{k, \ell}: T^{*} X \rightarrow \mathbb{R}$ defined by

$$
G_{k, \ell}(x, \xi)=g_{k, \ell}(|\xi|) .
$$

The flow $\varphi_{G_{k, \ell}, t}$ is related to the normalized geodesic flow $\gamma_{t}$ by the formula

$$
\varphi_{G_{k, \ell}, t}=\gamma_{g_{k, \ell}^{\prime}(|\xi|) t}
$$


(recall $|\xi|$ is constant under $\gamma_{t}$ ). In particular, $\varphi_{G_{k, \ell}, t}$ is the identity when $|\xi| \leq k$, and is equal to $\gamma_{t}$ when $|\xi| \geq \ell$.

We have the following reformulation of the previous lemma.

Lemma 6.3.3. There is $k>0$ and $\delta>0$ such that for all $\ell^{\prime}>k^{\prime}>k$ and $\delta^{\prime} \in(0, \delta]$, we have

$$
\varphi_{G_{k^{\prime}, \ell^{\prime}, \delta^{\prime}}}(\bar{L}) \cap \bar{\Lambda}_{\mathcal{S}}=L_{|\xi|<k^{\prime}} \cap \Lambda_{\mathcal{S}} .
$$

Proof. Immediate from the previous lemma and Lemma 6.2.1

6.4. Perturbations. We are now ready to describe how to perturb a collection of standard Lagrangians. In what follows, let $i$ denote an element of the index set $\mathbb{Z} /(d+1) \mathbb{Z}$.

Let $U_{i} \subset X$ be an open submanifold with closure $\bar{U}_{i} \subset X$ and boundary $\partial U_{i}=$ $\bar{U}_{i} \backslash U_{i}$. Fix a defining function $m_{i}: X \rightarrow \mathbb{R}$ for the closed subset $X \backslash U_{i}$, and let $f_{i}: U_{i} \rightarrow \mathbb{R}$ be the function $f_{i}=\log m_{i}$. Let $L_{i} \subset T^{*} X$ be the standard Lagrangian given by the graph of the differential of $f_{i}$. Choose a stratification of $X$ which refines the boundary $\partial U_{i} \subset X$, and let $\Lambda_{i} \subset T^{*} X$ denote the associated conical Lagrangian.

We will apply a sequence of Hamiltonian perturbations to the Lagrangians $L_{i}$ to put them in a good position. In order to satisfy the definition of the Fukaya category, the perturbations must be a positive normalized geodesic flow near infinity. Furthermore, the amounts $\delta_{i}>0$ of a normalized geodesic flow with which we move the $L_{i}$ near infinity must satisfy

$$
\left(\delta_{0}, \ldots, \delta_{d}\right) \in R \subset \mathbb{R}^{d+1},
$$

where $R$ is some fringed set. Because of this requirement, we will work backwards through the collection perturbing the Lagrangians in the order $L_{d}, \ldots, L_{0}$.

At the $i$ th stage, each of our perturbations will consist of two steps. (1) We will first variably dilate $L_{i}$ so that it becomes arbitrarily close to its associated conical Lagrangian $\Lambda_{i}$. (2) We will then gently perturb it near infinity in the direction of a positive geodesic flow. The first step will have three effects: (a) all of the intersections of the resulting Lagrangian with the previously perturbed Lagrangians will be near the zero section; (b) the height of the resulting Lagrangian will be less than that of the previously perturbed Lagrangians along certain critical contours; (c) intersections of the resulting Lagrangian with the associated conical Lagrangian of yet-to-be perturbed Lagrangians will be near the zero section. The second step will ensure that the first step is effective.

For each Lagrangian $L_{i}$, we organize the discussion of its perturbation into four parts:

- (Intersections) We first collect the other Lagrangians whose intersections with $L_{i}$ must be either dilated close to the zero section or perturbed away near infinity.

- (Dilation) The variable dilation to the zero section.

- (Separation) The small perturbation near infinity.

- (Conclusion) We finally organize the result so that we may proceed to the next Lagrangian.

Throughout, we fix a positive number $h>0$. We begin with the last Lagrangian in the collection. 
$L_{d}$ : (Intersections) Since there are no previously perturbed Lagrangians, our aim here is simpler than in general. Let $\Lambda_{\leq d} \subset T^{*} X$ be the conical Lagrangian

$$
\Lambda_{\leq d}=\bigcup_{j \leq d} \Lambda_{j} .
$$

To guarantee that intersections with the yet-to-be perturbed Lagrangians $L_{j}$, for $j<d$, can be dilated close to the zero section $T_{X}^{*} X$, we must dilate the intersection $L_{d} \cap \Lambda_{\leq d}$ close to $T_{X}^{*} X$.

(Dilation) By Lemma 6.3.1, the intersection $L_{d} \cap \Lambda_{\leq d}$ is compact.

Therefore we may choose $\sigma_{d}>0$ such that the standard dilation satisfies

$$
\left(\sigma_{d} \cdot L_{d}\right) \cap \Lambda_{\leq d} \subset N_{h / 2}\left(T_{X}^{*} X\right) .
$$

By compactness of the intersection and Lemma 6.2.1, we may choose $\eta_{d}>0$ so that

$$
\left(\sigma_{d} \cdot L_{d}\right) \cap \Lambda_{\leq d} \subset T^{*} X_{m_{d}>\eta_{d}} .
$$

To truncate this dilation near infinity, choose positive numbers $a_{d}<b_{d} \in\left(0, \eta_{d}\right)$ and a Hamiltonian function $D_{a_{d}, b_{d}}$ as in Lemma 6.2.3. The resulting variably dilated Lagrangian satisfies

$$
\varphi_{D_{a_{d}, b_{d}}, \delta_{d}}\left(L_{d}\right) \cap \Lambda_{\leq d} \subset T^{*} X_{m_{d}>\eta_{d},|\xi|<h / 2} .
$$

(Separation) Next, apply Lemma 6.3 .3 to the dilated Lagrangian $\varphi_{D_{a_{d}, b_{d}}, \delta_{d}}\left(L_{d}\right)$ and the conical Lagrangian $\Lambda_{\leq d}$. Let $M_{d}$ be the maximum of the length $\left|\sigma_{d} \cdot L_{d}\right|$ in the region $m_{d} \geq \eta_{d}$. We may choose $k_{d}>\max \left\{h, M_{d}\right\}$ and a Hamiltonian function $G_{k_{d}, \ell_{d}}$ such that for some $\epsilon_{d}>0$ the corresponding perturbation satisfies

$$
\varphi_{G_{k_{d}, \ell_{d}}, \delta_{d}}\left(\varphi_{D_{a_{d}, b_{d}}, \delta_{d}}\left(\bar{L}_{d}\right)\right) \cap \bar{\Lambda}_{\leq d}=\left(\varphi_{D_{a_{d}, b_{d}}, \delta_{d}}\left(\bar{L}_{d}\right)\right)_{|\xi|<k_{d}} \cap \Lambda_{\leq d} .
$$

(Conclusion) We set

$$
\begin{aligned}
\tilde{L}_{d} & =\varphi_{G_{k_{d}, \ell_{d}}, \delta_{d}}\left(\varphi_{D_{a_{d}, b_{d}}, \delta_{d}}\left(L_{d}\right)\right) \subset T^{*} X, \\
\tilde{U}_{d} & =X_{m_{d}>\eta_{d}} \subset X, \\
\tilde{\Gamma}_{d} & =\left(\tilde{L}_{d}\right)_{m_{d}>\eta_{d},|\xi|<k_{d}} \subset \tilde{L}_{d} .
\end{aligned}
$$

Note that $\tilde{\Gamma}_{d}$ is a graph over $\tilde{U}_{d}$. By construction, we have

$$
\begin{gathered}
\tilde{\Gamma}_{d}=\sigma_{d} \cdot\left(L_{d}\right)_{m_{d}>\eta_{d}}, \\
\tilde{L}_{d} \cap \bar{\Lambda}_{\leq d}=\tilde{\Gamma}_{d} \cap \Lambda_{\leq d} \subset N_{h / 2}\left(T_{X}^{*} X\right) .
\end{gathered}
$$

At an arbitrary step, we proceed as follows.

$L_{i}$ : (Intersections) Let $\tilde{L}_{>i} \subset T^{*} X$ be the union of the previously perturbed Lagrangians

$$
\tilde{L}_{>i}=\bigcup_{j>i} \tilde{L}_{j}
$$

We would like to dilate the intersection with $\tilde{L}_{>i}$ close to the zero section $T_{X}^{*} X$. We will not be able to move this intersection closer than the intersection $\tilde{L}_{>i} \cap \Lambda_{i}$, but at least this intersection is already close by induction.

Let $\Lambda_{\leq i} \subset T^{*} X$ be the conical Lagrangian

$$
\Lambda_{\leq i}=\bigcup_{j \leq i} \Lambda_{j} .
$$


To guarantee that intersections with the yet-to-be perturbed Lagrangians $L_{j}$, for $j<i$, can be dilated close to $T_{X}^{*} X$, we must dilate the intersection $L_{i} \cap \Lambda_{\leq i}$ close to $T_{X}^{*} X$.

Let $\Lambda_{>i} \subset T^{*} X$ be the union of conormals

$$
\Lambda_{>i}=\bigcup_{j>i} T_{\partial \tilde{U}_{j}}^{*} X .
$$

To guarantee that there is not unmanageable behavior along the boundaries of the previously defined open sets $\tilde{U}_{j}$, for $j>i$, we must dilate the intersection $L_{i} \cap \Lambda_{>i}$ close to $T_{X}^{*} X$.

We set

$$
\Lambda_{[i]}=\Lambda_{\leq i} \cup \Lambda_{>i}
$$

(Dilation) By induction, we have

$$
\overline{\tilde{L}}_{>i} \cap \bar{\Lambda}_{i} \subset N_{h / 2}\left(T_{X}^{*} X\right) .
$$

Since $L_{i}^{\infty} \subset \Lambda_{i}^{\infty}$, it follows that

$$
L_{i}^{\infty} \cap \tilde{L}_{>i}^{\infty}=\emptyset,
$$

and so the intersection $L_{i} \cap \tilde{L}_{>i}$ is also bounded. In addition, by Lemma 6.3.1, the intersection $L_{i} \cap \Lambda_{[i]}$ is compact.

Therefore we may choose $\sigma_{i}>0$ such that the standard dilation satisfies

$$
\begin{aligned}
& \left(\sigma_{i} \cdot L_{i}\right) \cap \tilde{L}_{>i} \subset N_{h}\left(T_{X}^{*} X\right), \\
& \left(\sigma_{i} \cdot L_{i}\right) \cap \Lambda_{[i]} \subset N_{h / 2}\left(T_{X}^{*} X\right) .
\end{aligned}
$$

Furthermore, for $\sigma_{i}>0$ sufficently small, we may arrange for

$$
\left|\sigma_{i} \cdot L_{i}\right|<\min _{j>i} M_{j} \text {, above the compact set }\left(\sigma_{i} \cdot L_{i}\right) \cap \Lambda_{>i},
$$

where $M_{j}$ denotes the maximum of the length $\left|\sigma_{i} \cdot L_{i}\right|$ in the region $m_{i} \geq \eta_{i}$. By compactness of the intersections and Lemma 6.2.1, we may choose $\eta_{i}>0$ so that

$$
\left(\sigma_{i} \cdot L_{i}\right) \cap\left(\tilde{L}_{>i} \cup \Lambda_{[i]}\right) \subset T^{*} X_{m_{i}>\eta_{i}} .
$$

To truncate this dilation near infinity, choose positive numbers $a_{i}<b_{i} \in\left(0, \eta_{i}\right)$ and a Hamiltonian function $D_{a_{i}, b_{i}}$ as in Lemma 6.2.3. The resulting variably dilated Lagrangian $\varphi_{D_{a_{i}, b_{i}}, \delta_{i}}\left(L_{i}\right)$ satisfies all of the properties derived above for the standard dilated Lagrangian $\sigma_{i} \cdot L_{i}$.

(Separation) Recall that we have

$$
L_{i}^{\infty} \cap \tilde{L}_{>i}^{\infty}=\emptyset .
$$

We apply Lemma 6.3.3 to the dilated Lagrangian $\varphi_{D_{a_{i}, b_{i}}, \delta_{i}}\left(L_{i}\right)$ and the conical Lagrangian $\Lambda_{[i]}$. Let $M_{i}$ be as above. We may choose $k_{i}>\max \left\{h, M_{i}\right\}$ and a function $G_{k_{i}, \ell_{i}}$ and an $\epsilon_{i}>0$ such that

$$
\varphi_{G_{k_{i}, \ell_{i}}, \delta_{i}}\left(\varphi_{D_{a_{i}, b_{i}}, \delta_{i}}\left(\bar{L}_{i}\right)\right) \cap \bar{\Lambda}_{[i]}=\left(\varphi_{D_{a_{i}, b_{i}}, \delta_{i}}\left(L_{i}\right)\right)_{|\xi|<k_{i}} \cap \Lambda_{[i]} .
$$

(Conclusion) We set

$$
\begin{aligned}
\tilde{L}_{i} & =\varphi_{G_{k_{i}, \ell_{i}}, \delta_{i}}\left(\varphi_{D_{a_{i}, b_{i}}, \delta_{i}}\left(L_{i}\right)\right) \subset T^{*} X, \\
\tilde{U}_{i} & =X_{m_{i}>\eta_{i}} \subset X, \\
\tilde{\Gamma}_{i} & =\left(\tilde{L}_{i}\right)_{m_{i}>\eta_{i},|\xi|<k_{i}} \subset \tilde{L}_{i} .
\end{aligned}
$$


Note that $\tilde{\Gamma}_{i}$ is a graph over $\tilde{U}_{i}$. By construction,

$$
\begin{aligned}
\tilde{\Gamma}_{i} & =\sigma_{i} \cdot\left(L_{i}\right)_{m_{i}>\eta_{i}}, \\
\overline{\tilde{L}_{i}} \cap \overline{\tilde{L}_{j}} & =\tilde{\Gamma}_{i} \cap \tilde{\Gamma}_{j} \subset N_{h}\left(T_{X}^{*} X\right), \text { for all } j>i, \\
\tilde{L}_{i} \cap \bar{\Lambda}_{[i]} & =\tilde{\Gamma}_{i} \cap \Lambda_{[i]} \subset N_{h / 2}\left(T_{X}^{*} X\right), \\
\left|\tilde{\Gamma}_{i}\right| & <\left|\tilde{\Gamma}_{j}\right| \text { wherever } \tilde{\Gamma}_{i} \cap T_{\partial \tilde{U}_{j}}^{*} X, \text { for all } j>i .
\end{aligned}
$$

By following this procedure, we arrive at the following.

Proposition 6.4.1. The collection of Lagrangians $\tilde{L}_{i}$, graphs $\tilde{\Gamma}_{i}$, and open sets $\tilde{U}_{i}$ satisfies the following:

(1) $\overline{\tilde{L}}_{i} \cap \overline{\tilde{L}}_{j}=\tilde{\Gamma}_{i} \cap \tilde{\Gamma}_{j}$, for $i \neq j$.

(2) $|\xi|^{2}$ has no critical points on $\tilde{L}_{i} \backslash \tilde{\Gamma}_{i}$.

(3) $\left(\tilde{U}_{i}, \tilde{\Gamma}_{i}\right)$ form a transverse collection in $\operatorname{Mor}(X)$; see Definition 4.6.4.

Proof. The last assertion is the only part left to check. By construction, the collection of boundaries $\partial \tilde{U}_{i}$ are transverse. To see if $\left(\tilde{U}_{i}, \tilde{\Gamma}_{i}\right)$ is transverse, we need only check that there is a metric for which the corresponding difference vector fields point in the appropriate inward and outward directions. Such a metric may be constructed locally wherever the level sets of the defining functions are transverse. By construction, at any places where transversality fails the relative sizes of the vector fields have been arranged to allow for a metric to be constructed.

6.5. Relation to Morse theory. The PSS isomorphism refers to an equivalence between Floer homology and singular homology, and appears in both Hamiltonian and Lagrangian Floer theory; see Section 3 of [1] for a recent discussion. In the context of Lagrangian graphs in the cotangent bundle of a compact manifold, Fukaya and Oh 9 extended this to an identification of the Morse and Fukaya $A_{\infty}$-categories by establishing an oriented diffeomorphism of the moduli spaces of gradient trees and holomorphic polygons involved in the definition of the higher composition maps. In the local setting of graphs over open sets with transverse boundaries, Kasturirangan and $\mathrm{Oh}$ 21, 22] prove an equivalence of the Morse and Floer chain complexes. In this section, we adapt the approach of Fukaya and Oh to prove an $A_{\infty}$-equivalence of Morse and Fukaya $A_{\infty}$-categories which include all standard objects. To do this, we first recall the theorem of Fukaya and $\mathrm{Oh}$ in its original form (with notation modified to agree with ours), and then adapt our situation to be able to apply their constructions.

Fukaya-Oh Theorem (9]). Let $(X, g)$ be a Riemannian manifold, and let $J_{\text {Sas }}$ be the canonical (Sasaki) almost complex structure on $T^{*} X$. Let $f=\left(f_{0}, \ldots, f_{d}\right)$ be a generic collection of functions on $X$, and let $\Gamma=\left(\Gamma_{d f_{0}}, \ldots, \Gamma_{d f_{d}}\right)$ be the graphs of their differentials. For sufficiently small $\epsilon>0$, there is an oriented diffeomorphism between the Morse moduli space of gradient trees of $f$ and the Fukaya moduli space of pseudoholomorphic disks (with respect to $J_{\text {Sas }}$ ) bounding the Lagrangians $\epsilon \Gamma$.

Recall that Proposition 6.4.1 of the preceding section provides, starting from a collection $L=\left(L_{0}, \ldots, L_{d}\right)$ of standard objects of $F u k\left(T^{*} X\right)$, a perturbed collection $\tilde{L}=\left(\tilde{L}_{0}, \ldots, \tilde{L}_{d}\right)$. Above the open set $\tilde{U}_{i}=X_{m_{i}>\eta_{i}}$, the perturbed object $\tilde{L}_{i}$ results from dilating the original object $L_{i}$, and thus in particular remains a graph

$$
\tilde{\Gamma}_{i}=\left(\tilde{L}_{i}\right)_{m_{i}>\eta_{i}}=\sigma_{i} \cdot\left(L_{i}\right)_{m_{i}>\eta_{i}} .
$$


Furthermore, all intersection points of the compactifications $\overline{\tilde{L}_{i}}$ occur among the $\tilde{\Gamma}_{i}$, and $|\xi|^{2}$ has no critical points on the complements $\tilde{L}_{i} \backslash \tilde{\Gamma}_{i}$. Finally, in the category $\operatorname{Mor}(X)$, we have a transverse collection of objects

$$
\tilde{\mathfrak{U}}=\left(\left(\tilde{U}_{0}, \tilde{f}_{0}\right), \ldots,\left(\tilde{U}_{d}, \tilde{f}_{d}\right)\right)
$$

where the graph of the differential $d \tilde{f}_{i}$ is precisely $\tilde{\Gamma}_{i}$. In what follows, we write $\tilde{\Gamma}$ for the collection of partial graphs $\left(\tilde{\Gamma}_{0}, \ldots, \Gamma_{d}\right)$.

We cannot apply the Fukaya-Oh theorem directly to the above situation for several reasons. First, the perturbed Lagrangians $\tilde{L}_{i}$ are noncompact and are no longer graphs. Moreover, as described in the previous section, we need to consider the conical almost complex structure $J_{\text {con }}$ which is only equal to the Sasaki almost complex structure $J_{S a s}$ near the zero section. Finally, the functions $\tilde{f}_{i}$ and corresponding graphs $\tilde{\Gamma}_{i}$ are defined only over the open sets $\tilde{U}_{i}$. Instead, we pursue the following strategy. Let us restrict our attention to the collection of bounded but partial graphs $\tilde{\Gamma}$. For small enough $\epsilon>0$, the local nature of the Fukaya-Oh theorem will give an identification of Fukaya and Morse moduli spaces for the dilated collection $\epsilon \tilde{\Gamma}$. Here we are using the fact that the conical almost complex structure $J_{c o n}$ is equal to the Sasaki almost complex structure $J_{S a s}$ near to the zero section. With this understood, we need only show that the Fukaya moduli spaces for $\epsilon \tilde{\Gamma}$ continue to calculate an $A_{\infty}$-structure quasi-isomorphic to that of the original unbounded but complete collection $\tilde{L}$. This comes down to showing compactness of the moduli spaces for $\epsilon \tilde{\Gamma}$ (and similarly, compactness of the moduli spaces providing continuation maps) as we vary the dilation parameter $\epsilon$.

Details of this approach follow below. Throughout we work with the conical almost complex structure $J_{c o n}$ and corresponding conical metric $g_{c o n}$ associated to a Riemannian metric on $X$. Our constructions will take place near the zero section where these structures agree with the respective Sasaki structures.

(1) (Area bounds) First, choose a small $\eta_{i}^{\prime}>\eta_{i}$, and consider the level-set

$$
X_{m_{i}=\eta_{i}^{\prime}} \subset X \text {. }
$$

Choose a small $\delta_{i, h}>0$, and define the annulus-like open set

$$
S_{i} \subset X
$$

to consist of all points whose distance to the level-set $X_{m_{i}=\eta_{i}^{\prime}}$ is less than $\delta_{i, h}$. Consider the annulus-like partial graph

$$
G_{i}=\tilde{\Gamma}_{i} \cap \pi^{-1}\left(S_{i}\right)
$$

Finally, choose a very small $\delta_{i, v}>0$, and define the tube-like open set

$$
T_{i} \subset T^{*} X
$$

to be the union of the vertical balls $B_{\delta_{i, v}}^{v} \subset T^{*} X$ of radius $\delta_{i, v}$ centered at points of $G_{i}$. Here by the vertical ball $B_{\delta_{i, v}}^{v}$ around a covector $(x, \xi) \in T^{*} X$, we mean the ball in the fiber $T_{x}^{*} X$ centered at $\xi$.

By construction, for small enough $\delta_{i, h}>0$, the boundary of the partial graphs $G_{i}$ decomposes as a disjoint union of manfolds

$$
\partial G_{i}=H_{i}^{\text {in }} \cup H_{i}^{\text {out }}
$$


where $\left.m_{i}\right|_{H_{i}^{i n}}>\eta_{i}^{\prime}$ and $\left.m_{i}\right|_{H_{i}^{\text {out }}}<\eta_{i}^{\prime}$. Furthermore, for small enough $\delta_{i, v}>0$, there will be no interaction among the tubes:

$$
T_{i} \cap T_{j}=\emptyset, \text { for } i \neq j .
$$

Our aim is to use the relatively compact region $T_{i}$ to construct an area bound on holomorphic disks. An important wrinkle is that we would like the bound to behave well with respect to dilations of $\tilde{L}_{i}$ together with $T_{i}$ towards the zero section. The precise statement we need is contained in the following monotonicity bound from [35.

Lemma 6.5.1. There exist constants $R_{i}, a_{i}>0$ such that the following holds for any $0<\epsilon \leq 1$.

Consider a holomorphic map $u:(D, \partial D) \rightarrow\left(\epsilon T_{i}, \epsilon G_{i}\right)$. Then for any ball $B_{r} \subset$ $\epsilon T_{i}$ of radius $r<\epsilon R_{i}$ such that $u(D)$ contains the center of $B_{r}$, we have

$$
\operatorname{Area}\left(u(D) \cap B_{r}\right)>a_{i} r^{2} .
$$

Proof. For fixed $0<\epsilon \leq 1$, the assertion follows from Proposition 4.7.2 of [35] (note that Sikorav's proofs of 4.3.1(ii) and 4.7.2(ii) are entirely local - only the bounding constants are global in nature).

What remains is the assertion that the bound can be achieved uniformly with respect to dilation by $\epsilon$. But the family of graphs $\epsilon G_{i}$ extends to a compact family including $\epsilon=0$, where we simply take the zero section itself. Thus all of the controls on the geometric complexity required to apply the area bound of [35] can be achieved uniformly with respect to $\epsilon$.

The above bounds for $\epsilon=1$ allow us to control where disks with boundary along $\tilde{L}$ can go. More precisely, we can fix a small radius $0<\mathfrak{r}_{i}<R_{i}$ such that any ball $B_{\mathfrak{r}_{i}}$ of radius $\mathfrak{r}_{i}$ centered at a point of $\left(G_{i}\right)_{m_{i}=\eta^{\prime}}$ fits inside $T_{i}$. Then by variably dilating each $\tilde{L}_{i}$ while fixing $T_{i}$, we can arrange that any disk along the resulting collection with a fixed number $d$ of marked points has area less than the minimum of the above bounds $a_{i} \mathfrak{r}_{i}^{2}$. Thus the boundaries of the disks cannot pass through the regions $G_{i}$ and so must lie on the variably dilated $\tilde{\Gamma}_{i}$.

(2) (Uniform dilation) Next, we take advantage of the homogeneity of the above area bounds to see that we need not restrict ourselves to variable dilations of the $\tilde{L}_{i}$, but can in fact dilate the entire $\tilde{L}_{i}$ uniformly. The main point is that the argument of the preceeding paragraph, which applies to $\epsilon=1$, is robust enough to allow the $T_{i}$ to be dilated to $\epsilon T_{i}$ as well.

To begin, we refine some of the choices made in the previous step. First, fix any $0<\delta_{i, h}^{\prime}<\delta_{i, h}$, and consider the smaller annulus-like region

$$
S_{i}^{\prime} \subset X
$$

consisting of all points whose distance to the level-set $X_{m_{i}=\eta_{i}^{\prime}}$ is less than $\delta_{i, h}^{\prime}$. Similarly, consider the annulus-like partial graph

$$
G_{i}^{\prime}=\tilde{\Gamma}_{i} \cap \pi^{-1}\left(S_{i}^{\prime}\right) .
$$

For any such $\delta_{i, h}^{\prime}$, we can find $0<\mathfrak{r}_{i}<R_{i}$ such that for any ball $B_{\mathfrak{r}_{i}} \subset T^{*} X$ of radius $\mathfrak{r}_{i}$ centered at any point of $G_{i}^{\prime}$, we have

$$
B_{\mathfrak{r}_{i}} \subset T_{i} .
$$


Fix such a radius $\mathfrak{r}_{i}$ and also fix the area bound $a_{i}$ given by Lemma 6.5.1. As explained above, after variably dilating each $\tilde{L}_{i}$ while fixing the $T_{i}$, we may assume that the area of any disk along the resulting collection satisfies

$$
\operatorname{Area}(u(D))<a_{i} \mathfrak{r}_{i}^{2} .
$$

Now, consider the absolute dilation $\epsilon \tilde{L}$ for any $0<\epsilon \leq 1$. We claim that for any holomorphic map $u:(D, \partial D) \rightarrow\left(T^{*} X, \epsilon \tilde{L}\right)$ with a fixed number of marked points $d$, the boundary $u(\partial D)$ must in fact lie in $\epsilon \tilde{\Gamma}$. Suppose otherwise, and consider a boundary path $u(C)$ in $\epsilon G_{i}$ traversing from $\epsilon H_{i}^{i n}$ to $\epsilon H_{i}^{\text {out }}$. By construction, through subdividing $S_{i}^{\prime}$ and considering the induced subdivision of $\epsilon T_{i}$, we can find $1 / \epsilon$ disjoint balls $B_{\epsilon \mathfrak{r}_{i}} \subset \epsilon T_{i}$ of radius $\epsilon \mathfrak{r}_{i}$ such that the path contains the centers of the $B_{\epsilon \mathfrak{r}_{i}}$. Thus the lemma gives the area bound

$$
\operatorname{Area}\left(u(D) \cap \epsilon T_{i}\right)>\epsilon a_{i} \mathfrak{r}_{i}^{2} .
$$

Since the possible area of disks with boundary on the collection also scales linearly with the dilation paramter $\epsilon$ ( $c f$. Lemma 5.2.6), we conclude that the initial area bound for $\epsilon=1$ implies that the boundary $u(\partial D)$ must lie in $\epsilon \tilde{\Gamma}$.

(3) (Application of the Fukaya-Oh theorem) Finally, we apply the Fukaya-Oh theorem. The key observation to make is that the proof of Fukaya-Oh given in 9 is local in the following sense. Given a gradient tree in $X$ for the functions $f$, the corresponding holomorphic disk in $T^{*} X$ for the graphs $\epsilon \Gamma$ will be in a neighborhood of the gradient tree such that the size of the neighborhood goes to zero as $\epsilon$ goes to zero. Conversely, any such holomorphic disk will arise in this way.

More precisely, starting from a gradient tree, Fukaya and Oh first construct an approximate holomorphic disk $w_{\epsilon}$. The distance between the gradient tree and $w_{\epsilon}$ goes to zero as $\epsilon \rightarrow 0$. Next, an actual solution is proven to exist nearby in the $L^{\infty}$-topology. The actual solution (in the notation of [9]) has the form $\exp _{w_{\epsilon}}(Q \eta)$. The point is that by Theorem 9.1 of [9], for all $\delta>0$ there exists an $\epsilon>0$ such that for all $0<\epsilon^{\prime}<\epsilon$, one has $\|Q \eta\|_{L^{\infty}}<\delta$. In other words, the distance between the actual solution and the gradient tree can be made arbitrarily small as long as $\epsilon$ goes to zero.

We conclude that by dilating our partial graphs $\tilde{\Gamma}_{i}$ over the open sets $\tilde{U}_{i}$ uniformly close to the zero section, any gradient tree for the collection $\tilde{f}$ will correspond to a holomorphic disk with boundary on the $\tilde{\Gamma}_{i}$. Finally, the area bounds from the previous step implies that such holomorphic disks are the only ones to be considered. We thus have arrived at our desired result.

Theorem 6.5.2. There is an $A_{\infty}$-quasi-equivalence between $\operatorname{Mor}(X)$ and the full subcategory of $F u k\left(T^{*} X\right)$ generated by the standard objects $L=\Gamma_{d f}$ over open sets $U \subset X$, where $f: U \rightarrow \mathbb{R}$ is given by $f=\log m$, and $m: X \rightarrow \mathbb{R}_{\geq 0}$ is a defining function for the complement $X \backslash U$.

\section{ARBitRARY STANDARD OBJECTS}

For future applications, it is useful to know where the embedding takes other objects and morphisms. In particular, we would like to know not only where it takes standard sheaves on open submanifolds, but also standard sheaves on arbitrary submanifolds. As discussed in the Introduction, one approach to this problem is to express standard sheaves on arbitrary submanifolds in terms of standard sheaves on open submanifolds, and then to check what the relevant distinguished triangles 
of constructible sheaves look like under the embedding. This requires identifying certain cones in the Fukaya category with symplectic surgeries. Rather than taking this route, we will instead show in this section that we may explicitly extend the domain of the embedding to include standard sheaves on arbitrary submanifolds and morphisms between them.

We will follow very closely the steps used to define the embedding in the preceding sections. First, we will interpret the dg category $S h(X)$ of constructible sheaves in terms of a category $S u b(X)$ whose objects are submanifolds (equipped with certain defining functions) and whose morphisms are complexes of relative de Rham forms (on certain open submanifolds with hypercorners). Next, we will interpret the category $\operatorname{Sub}(X)$ in terms of an extended version of the category $\operatorname{Mor}(X)$ built out of Morse theory. Finally, we will explain how the work of Fukaya-Oh may be adapted to identify $\operatorname{Mor}(X)$ with a full subcategory of the Fukaya category $F u k\left(T^{*} X\right)$. Because of the amount of overlap with the preceding sections, we will only explain the new wrinkles which arise and not repeat all details.

Before continuing, we state where the embedding takes the standard sheaf $i_{*} \mathcal{L}_{Y}$ associated to a local system $\mathcal{L}_{Y}$ on an arbitrary submanifold $i: Y \hookrightarrow X$. Suppose that we are given a defining function $m: X \rightarrow \mathbb{R}_{\geq 0}$ for the boundary $\partial Y \subset X$. Recall that we define the standard Lagrangian $L_{Y, m} \subset T^{*} X$ to be the fiberwise sum

$$
L_{Y, m}=T_{Y}^{*} X+\Gamma_{d \log m}
$$

where $T_{Y}^{*} X \subset T^{*} X$ is the conormal bundle to $Y$ and $\Gamma_{d \log m} \subset T^{*} X$ is the graph of the differential of $\log m$. As explained in Section [5.3, it comes equipped with a canonical brane structure $b$, along with a flat bundle $\mathcal{E}=\pi^{*}\left(\mathcal{L}_{Y} \otimes \operatorname{or}_{X} \otimes o r_{Y}^{-1}\right)$. We write $L_{Y, m, \mathcal{L}_{Y}}$ for the corresponding object of $F u k\left(T^{*} X\right)$. The main consequence of this section is the following.

Theorem 7.0.3. Under the quasi-embedding $S h(X) \hookrightarrow T w F u k\left(T^{*} X\right)$, the image of the standard sheaf $i_{*} \mathcal{L}_{Y}$ is canonically isomorphic to the standard brane $L_{Y, m, \mathcal{L}_{Y}}$.

In what follows, we limit the discussion to the case of trivial local systems since the arbitrary case is no more difficult. This will help streamline the exposition for example, we write $L_{Y, m}$ for the object $L_{Y, m, \mathcal{L}_{Y}}$ when $\mathcal{L}_{Y}$ is trivial.

7.1. Submanifold category. In Sections 4.4 and 4.5. we introduced the dg category $\operatorname{Open}(X)$. The results of this section generalize that discussion.

We define a dg category $\operatorname{Sub}(X)$ as follows. The objects of $S u b(X)$ are triples $(Y, m, n)$, where $Y \subset X$ is a submanifold, $m: X \rightarrow \mathbb{R}_{\geq 0}$ is a defining function for its boundary $\partial Y \subset X$, and $n: X \rightarrow \mathbb{R}_{\geq 0}$ is a defining function for its closure $\bar{Y} \subset X$. To define the complex of morphisms from an object $\mathfrak{Y}_{0}=\left(Y_{0}, m_{0}, n_{0}\right)$ to an object $\mathfrak{Y}_{1}=\left(Y_{1}, m_{1}, n_{1}\right)$, we introduce some perturbations. It will be clear that the choices range over a contractible set, and that they can be made compatible for any finite collection of objects. We will use the following general statement repeatedly.

Lemma 7.1.1. Let $\mathfrak{Y}=(Y, m, n)$ be an object of $S u b(X)$, and let $\Lambda \subset T^{*} X$ be an arbitrary conical Lagrangian. There is a fringed set $R \subset \mathbb{R}^{2}$ such that for all $(\eta, \kappa) \in R$, we have

(1) $\eta$ is not a $\Lambda$-critical value of $m$,

(2) $\kappa$ is not a $\Lambda$-critical value of $n$, 
(3) $(\eta, \kappa)$ is not a $\Lambda$-critical value of $m \times n$.

Proof. Critical values form a closed $\mathcal{C}$-subset, and their complement is dense.

First, fix a Whitney stratification $\mathcal{S}_{0}$ of $X$ compatible with $Y_{0} \subset X$, and let $\Lambda_{\mathcal{S}_{0}} \subset T^{*} X$ be the conical conormal set associated to $\mathcal{S}_{0}$. Apply the preceding lemma to $\mathfrak{Y}_{1}=\left(Y_{1}, m_{1}, n_{1}\right)$ and $\Lambda_{\mathcal{S}_{0}}$ to obtain a fringed set $R_{1} \subset \mathbb{R}^{2}$. For any $\left(\eta_{1}, \kappa_{1}\right) \in R_{1}$, let $T_{1} \subset X$ be the open submanifold with corners

$$
T_{1}=\left\{x \in X \mid m_{1}(x)>\eta_{1}, n_{1}(x)<\kappa_{1}\right\} .
$$

We think of $T_{1}$ as a tube around $Y_{1}$. We refer to the codimension one boundary piece

$$
E_{1}=\left\{x \in X \mid m_{1}(x)=\eta_{1}, n_{1}(x)<\kappa_{1}\right\}
$$

as the end of $T_{1}$, and the codimension one boundary piece

$$
S_{1}=\left\{x \in X \mid m_{1}(x)>\eta_{1}, n_{1}(x)=\kappa_{1}\right\}
$$

as the side of $T_{1}$.

Next, for any $\left(\eta_{1}, \kappa_{1}\right) \in R_{1}$, fix the Whitney stratification $\mathcal{S}_{\left(\eta_{1}, \kappa_{1}\right)}$ of $X$ given by $T_{1}$, the codimension one pieces of its boundary, the corners of its boundary, and the complement of its closure. Now let $\Lambda_{\mathcal{S}_{\left(\eta_{1}, \kappa_{1}\right)}}$ be the conical conormal set associated to $\mathcal{S}_{\left(\eta_{1}, \kappa_{1}\right)}$. Apply the lemma to $\mathfrak{Y}_{0}=\left(Y_{0}, m_{0}, n_{0}\right)$ and $\Lambda_{\mathcal{S}_{1}}$ to obtain a fringed set $R_{0} \subset \mathbb{R}^{2}$. For any $\left(\eta_{0}, \kappa_{0}\right) \in R_{0}$, let $T_{0} \subset X$ be the open tube

$$
T_{0}=\left\{x \in X \mid m_{0}(x)>\eta_{0}, n_{0}(x)<\kappa_{0}\right\},
$$

with end

$$
E_{0}=\left\{x \in X \mid m_{0}(x)=\eta_{0}, n_{0}(x)<\kappa_{0}\right\}
$$

and side

$$
S_{0}=\left\{x \in X \mid m_{0}(x)>\eta_{0}, n_{0}(x)=\kappa_{0}\right\} .
$$

We will also need the relative dualizing objects $\omega_{T_{0} / Y_{0}}, \omega_{T_{1} / Y_{1}}$. To construct these, choose retracting fibrations of pairs

$$
\begin{aligned}
& \bar{\pi}_{0}:\left(T_{0} \cup E_{0}, E_{0}\right) \rightarrow\left(\left(T_{0} \cup E_{0}\right) \cap Y_{0}, E_{0} \cap Y_{0}\right), \\
& \bar{\pi}_{1}:\left(T_{1} \cup E_{1}, E_{1}\right) \rightarrow\left(\left(T_{1} \cup E_{1}\right) \cap Y_{1}, E_{1} \cap Y_{1}\right),
\end{aligned}
$$

consider the restrictions $\pi_{0}=\left.\bar{\pi}_{0}\right|_{T_{0}}, \pi_{1}=\left.\bar{\pi}_{1}\right|_{T_{1}}$, and define

$$
\omega_{T_{0} / Y_{0}}=\pi_{0}^{!} \mathbb{C}_{Y_{0}}, \quad \omega_{T_{1} / Y_{1}}=\pi_{1}^{!} \mathbb{C}_{Y_{1}} .
$$

Concretely, $\omega_{T_{0} / Y_{0}}, \omega_{T_{1} / Y_{1}}$ are canonically isomorphic to the local systems (placed in degrees - codim $\left.Y_{0},-\operatorname{codim} Y_{1}\right)$ on $T_{0}, T_{1}$ of relative orientations along the fibers of $\pi_{0}, \pi_{1}$ respectively.

Finally, we define the morphisms in the dg category $S u b(X)$ to be given by the relative de Rham complex

$$
\operatorname{hom}_{\operatorname{Sub}(X)}\left(\mathfrak{Y}_{0}, \mathfrak{Y}_{1}\right)=\left(\Omega\left(T_{0} \cap T_{1}, E_{0} \cup S_{1} ; \omega_{T_{0} / Y_{0}}^{-1} \otimes \omega_{T_{1} / Y_{1}}\right), d\right) .
$$

Given a finite collection of objects of $S u b(X)$, we may generalize the above perturbation procedure in order to define the composition of morphisms as the wedge product of differential forms. 
Proposition 7.1.2. For submanifolds $i_{0}: Y_{0} \hookrightarrow X, i_{1}: Y_{1} \hookrightarrow X$, we have a canonical quasi-isomorphism

$$
\operatorname{hom}_{S h(X)}\left(i_{0 *} \mathbb{C}_{Y_{0}}, i_{1 *} \mathbb{C}_{Y_{1}}\right) \simeq\left(\Omega\left(T_{0} \cap T_{1}, E_{0} \cup S_{1} ; \omega_{T_{0} / Y_{0}}^{-1} \otimes \omega_{T_{1} / Y_{1}}\right), d\right) .
$$

The composition of morphisms coincides with the wedge product of differential forms.

Proof. The proof is similar to the proofs of Sections 4.4 and 4.5

Consider the inclusions

$$
T_{0} \stackrel{s_{0}}{\hookrightarrow} T_{0} \cup S_{0} \stackrel{e_{0}}{\hookrightarrow} X, \quad T_{1} \stackrel{s_{1}}{\hookrightarrow} T_{1} \cup S_{1} \stackrel{e_{1}}{\hookrightarrow} X .
$$

Then by de Rham's theorem, we have a quasi-isomorphism

$$
\operatorname{hom}_{S h(X)}\left(e_{0 *} s_{0 !} \omega_{T_{0} / Y_{0}}, e_{1 *} s_{1} ! \omega_{T_{1} / Y_{1}}\right) \simeq\left(\Omega\left(T_{0} \cap T_{1}, E_{0} \cup S_{1} ; \omega_{T_{0} / Y_{0}}^{-1} \otimes \omega_{T_{1} / Y_{1}}\right), d\right) .
$$

One may identify the left hand side of this quasi-isomorphism with that of the proposition using standard identities as in Lemma 4.4.1, and repeated applications of the Thom isotopy lemma as in Lemmas 4.5.1 and 4.5.2. We leave the details including the last assertion to the interested reader.

By the preceding proposition, we may define a dg functor

$$
P: S u b(X) \rightarrow S h(X)
$$

by sending an object $\mathfrak{Y}=(Y, m, n)$ to the standard sheaf $i_{*} \mathbb{C}_{Y}$, where $i: Y \hookrightarrow X$ is the inclusion. The induced dg functor on twisted complexes $\operatorname{TwP}: \operatorname{Tw} \operatorname{Sub}(X) \rightarrow$ $\operatorname{Sh}(X)$ is a quasi-equivalence.

7.2. Morse theory interpretation. In Section 4.6, we introduced the $A_{\infty}$-category $\operatorname{Mor}(X)$ and showed it is quasi-equivalent to $\operatorname{Open}(X)$. The results of this section generalize that discussion.

We extend the definition of $\operatorname{Mor}(X)$ as follows. As with $\operatorname{Sub}(X)$, we take the objects of $\operatorname{Mor}(X)$ to be triples $(Y, m, n)$, where $Y \subset X$ is a submanifold, $m: X \rightarrow$ $\mathbb{R}_{\geq 0}$ is a defining function for its boundary $\partial Y \subset X$, and $n: X \rightarrow \mathbb{R}_{\geq 0}$ is a defining function for its closure $\bar{Y} \subset X$. To define the complex of morphisms from an object $\mathfrak{Y}_{0}=\left(Y_{0}, m_{0}, n_{0}\right)$ to an object $\mathfrak{Y}_{1}=\left(Y_{1}, m_{1}, n_{1}\right)$, we introduce some constructions refining those of the previous section.

To refine the procedure of the preceding section, we first fix $\left(\eta_{1}, \bar{\kappa}_{1}\right) \in R_{1}$ and consider the function

$$
f_{1}=\log m_{1}-\log \left(\bar{\kappa}_{1}-n_{1}\right) .
$$

For any positive $\kappa_{1}<\bar{\kappa}_{1}$, we have the open tube

$$
T_{1}=\left\{x \in X \mid m_{1}(x)>\eta_{1}, n_{1}(x)<\kappa_{1}\right\} .
$$

If $\kappa_{1}$ is sufficiently close to $\bar{\kappa}_{1}$, then there is a convex open set of Riemannian metrics on $X$ for which the gradient $\nabla f_{1}$ is inward pointing along the end $E_{1}$ and outward pointing along the side $S_{1}$.

We next proceed similarly and choose $\left(\eta_{0}, \bar{\kappa}_{0}\right) \in R_{0}$ and consider the function

$$
f_{0}=\log m_{0}-\log \left(\bar{\kappa}_{0}-n_{0}\right) .
$$

For any positive $\kappa_{0}<\bar{\kappa}_{0}$, we have the open tube

$$
T_{0}=\left\{x \in X \mid m_{0}(x)>\eta_{0}, n_{0}(x)<\kappa_{0}\right\} .
$$

For sufficiently small positive $\eta_{0}$, and $\kappa_{0}$ sufficiently close to $\bar{\kappa}_{0}$, there is a convex open set of Riemannian metrics on $X$ for which the gradient $\nabla f_{1}-\nabla f_{0}$ is inward 
pointing along the end $E_{0} \cap T_{1}$ and outward pointing along the side $S_{0} \cap T_{1}$. To ensure analogous but opposite behavior along the end $T_{0} \cap E_{1}$ and the side $T_{0} \cap S_{1}$, we proceed as follows. By moving $f_{0}$ to a new function $\widetilde{f}_{0}$, we may arrange it so that there is an open convex set of Riemannian metrics on $X$ for which the gradient $\nabla f_{1}-\nabla \widetilde{f}_{0}$ is outward pointing along the end $T_{0} \cap E_{1}$ and inward pointing along the side $T_{0} \cap S_{1}$. Furthermore, for an open convex set of Riemannian metrics on $X$, we continue to have that $\nabla f_{1}-\nabla \tilde{f}_{0}$ is inward pointing along the end $E_{0} \cap T_{1}$ and outward pointing along the side $S_{0} \cap T_{1}$.

Finally, we choose small perturbations of our functions and metric, and define the morphisms of $\operatorname{Mor}(X)$ to be the Morse complex

$$
\operatorname{hom}_{M o r(X)}\left(\mathfrak{Y}_{0}, \mathfrak{Y}_{1}\right)=\left(\bigoplus_{p \in C r\left(T_{0} \cap T_{1}, f_{1}-\widetilde{f}_{0}\right)} \mathcal{H o m}\left(\left.\omega_{T_{0} / Y_{0}}\right|_{p},\left.\omega_{T_{1} / Y_{1}}\right|_{p}\right), m_{M o r(X)}^{1}\right) .
$$

The verification that this is a well-defined complex is similar to Lemma 4.6.3. As usual, to define the higher compositions, one generalizes the above procedure sequentially for a finite collection of objects. The details of this are no more complicated than in other contexts considered earlier. Similarly, the fact that we obtain an $A_{\infty}$-category follows from homological perturbation theory along the same lines as the arguments of Section 4.6.4. In addition, as in Section 4.6.4, homological perturbation theory also provides an $A_{\infty}$-quasi-equivalence

$$
M: \operatorname{Sub}(X) \rightarrow \operatorname{Mor}(X) .
$$

7.3. Identification with standard branes. In Sections 6.4 and 6.5 we explained how to calculate morphisms in the Fukaya category among standard branes associated to open submanifolds. In this section, we adapt that discussion to the case of standard branes associated to arbitrary submanifolds.

Recall that given a defining function $m: X \rightarrow \mathbb{R}_{>0}$ for the boundary $\partial Y \subset X$, we define the standard Lagrangian $L_{Y, m} \subset T^{*} X$ to be the fiberwise sum

$$
L_{Y, m}=T_{Y}^{*} X+\Gamma_{d \log m},
$$

where $T^{*} X_{Y} \subset T^{*} X$ is the conormal bundle to $Y$ and $\Gamma_{d \log m} \subset T^{*} X$ is the graph of the differential of $\log m$. It comes equipped with a canonical flat bundle and brane structure and thus may be considered as an object of $F u k\left(T^{*} X\right)$.

7.3.1. Perturbations. We first explain the necessary modifications to the perturbation procedure of Section 6.4. Recall that our perturbations were made up of two steps: a variable dilation followed by a separation at infinity. In our current setting, this may not be enough to guarantee that the height of our Lagrangians will be small enough along certain critical contours. Namely, it may not hold that the pairwise differences of our Lagrangians provide vector fields with prescribed inward and outward behavior along the codimension one boundary components of the intersections of certain open tubes. Thus we will add a third independent step: a final variable dilation. Before explaining this, we first comment on the only substantive change in the first two steps.

The first step involving a variable dilation remains the same. But we will change the second step as follows. Rather than using it solely to separate Lagrangians near infinity, we will also use it to tilt our standard branes so that they become very close to being graphs over open subsets. We will explain this in the setting of a 
single standard brane $L_{Y, m}$ and leave it to the reader to repeat the arguments of Section 6.4 using this version of the separation step.

We reinterpret the separation flow as follows. Fix large positive numbers $k<$ $\ell \in(0, \infty)$, and choose a decreasing function $b_{k, \ell}: \mathbb{R} \rightarrow \mathbb{R}$ satisfying the following:

$$
b_{k, \ell}(r)= \begin{cases}1, & \text { for } r \leq k, \\ 0, & \text { for } r \geq \ell\end{cases}
$$

It will be convenient to consider the translation of the cotangent bundle $T^{*} X$ where the zero section is given by the scaled Lagrangian

$$
Z=b_{k, \ell}(|\xi|) \cdot \Gamma_{d \log m} .
$$

Note that though $\Gamma_{d \log m}$ is a singular graph, the function $b_{k, \ell}(|\xi|)$ is constructed to be zero near the singularities. Thus the section $Z$ is a well-defined graph over all of $X$. Observe that fiberwise addition by $Z$ is a symplectomorphism.

Fix $\bar{\kappa}>0$, and consider the function $h: \mathbb{R} \rightarrow \mathbb{R}$ defined by

$$
h(r)=\int_{0}^{r} \frac{-1+\sqrt{1+4 r^{2} \bar{\kappa}}}{2 r}
$$

for $r \neq 0$, and $h(0)=0$. Note that $h$ is differentiable. Consider the Hamiltonian

$$
H(x, \xi)=h(|\xi-Z|),
$$

where we take the length of the fiberwise difference. The associated vector field $v_{H}$ is in the direction $v_{\theta}$ with length $h^{\prime}(|\xi-Z|)$. Thus the flow $\varphi_{H, t}$ associated to $v_{H}$ is nothing more than a rescaled version of the geodesic flow.

The point of choosing $h$ as we have is the following. Applying $\varphi_{H, t}$ to the standard Lagrangian $\Gamma_{d \log m}$ leads to a perturbation with similar characteristics as that considered in Section 6.3. Applying $\varphi_{H, t}$ to the standard Lagrangian $L_{Y, m}$ produces a perturbed Lagrangian which is a graph over an open tube $T$ around $Y$. To be more precise, recall from the preceding sections the construction of the tube $T$ associated to an object $\mathfrak{Y}=(Y, m, n)$. Namely, we fix $(\eta, \bar{\kappa})$ in the fringed set $R$, and then for any positive $\kappa<\bar{\kappa}$, we form the open tube

$$
T=\{x \in X \mid m(x)>\eta, n(x)<\kappa\} .
$$

Now assume that within the region $m \geq \eta$ the defining function $n$ is equal to half the squared-distance from $Y$. (Note that this is not a significant constraint, since we may choose $m$ and $\eta$ independently beforehand.) Then over the tube $T$, the unit time perturbation $\varphi_{H, 1}\left(L_{Y, m}\right)$ will be the graph of the differential of the function

$$
f=\log m-\log (\bar{\kappa}-n) .
$$

In the next section, we will use this compatibility with the previously defined Morse category $\operatorname{Mor}(X)$ in order to see that calculations in $\operatorname{Mor}(X)$ agree with those in the Fukaya category $F u k\left(T^{*} X\right)$. Note that although the above perturbation is only an asymptotically normalized geodesic flow, it may easily be modified to be precisely normalized geodesic flow near infinity without changing any of its other properties.

Finally, we add a third step to our perturbation procedure to ensure that the height of our Lagrangians will be small enough along certain critical contours. Namely, we must further move our perturbed Lagrangian to guarantee that in analogy with Proposition 6.4.1, when we consider multiple standard Lagrangians, their 
pairwise differences will have the correct behavior (inward and outward pointing) along the boundaries of the intersections of the corresponding tubes.

7.3.2. Relation to Morse theory. The arguments of Section 6.5 extend directly to this setting. To simplify things, we may work with objects $\mathfrak{Y}=(Y, m, n)$ such that $n$ is equal to half the squared-distance from the closure $\bar{Y} \subset X$ away from the boundary $\partial Y \subset \bar{Y}$. More precisely, we may assume $n$ is equal to half the squared-distance from $Y$ within the region $m \geq \eta$, where $(\eta, \kappa)$ is in the fringed set $R$ for small enough $\kappa$. Then as we have seen, our Morse perturbations and Fukaya perturbations are compatible. To control the possible holomorphic polygons, we proceed similarly as in Section 6.5. The only amendment is that here for each object $\mathfrak{Y}=(Y, m, n)$, when we show that polygons do not escape as in Lemma 6.5.1. we begin the arguments with a region $S$ which is a small neighborhood of the hypersurface with corners

$$
X_{m=\eta^{\prime}, n \leq \kappa^{\prime}} \cup X_{m \geq \eta^{\prime}, n=\kappa^{\prime}} .
$$

Then as before, applying the theorem of Fukaya and Oh provides the desired identification of moduli spaces. In conclusion, we obtain an $A_{\infty}$-quasi-embedding $\operatorname{Mor}(X) \hookrightarrow F u k\left(T^{*} X\right)$ extending that of Section 6.5. and that takes the standard object $\mathfrak{Y}=(Y, m, n)$ to the standard brane $L_{Y, m}$.

7.4. Other objects. Here we informally mention another class of objects of $S h(X)$ which also go to Lagrangians under our quasi-embedding: the so-called tilting perverse sheaves. These may be thought of as extensions of flat vector bundles on submanifolds with boundary conditions somewhere between the standard and costandard extensions. While the intersection cohomology or intermediate extension is cohomologically between the standard and costandard extensions, the tilting extension (if it exists) is geometrically between the two. To understand this, note that one can view the standard Lagrangian $L_{Y, m}$ as giving a vector field on $Y$ which is everywhere inward pointing along $\partial Y$, and similarly, the costandard Lagrangian $-L_{Y, m}$ as giving a vector field which is everywhere outward pointing. The Lagrangians associated to tilting perverse sheaves give vector fields which are sometimes inward and sometimes outward pointing over prescribed parts of the boundary.

Rather than further developing this picture here, we content ourselves with giving an example and picking up the discussion elsewhere. Consider the complex line $\mathbb{C} \simeq \mathbb{R}^{2}$ with coordinate $z$, and let $i: U \hookrightarrow X$ be the open subset $U=\{z \in \mathbb{C} \mid z \neq$ $0\}$. Given the defining function $m(z)=|z|^{2} / 2$ for the point $0 \in \mathbb{C}$, the standard Lagrangian corresponding to the standard sheaf $i_{*} \mathbb{C}_{U}$ is given by the graph of the real part of $d z / z$. Similarly, the costandard Lagrangian corresponding to the costandard sheaf $i_{1} \mathbb{C}_{U}$ is given by the graph of the real part of $-d z / z$. The graph of the real part of $d z / z^{n}$, for $n \geq 2$, is a Lagrangian corresponding to a tilting perverse sheaf. In particular, for $n=2$, it corresponds to the indecomposable tilting extension of the constant sheaf on $U$.

\section{ACKNOWLEDGMENTS}

We have benefited greatly from discussions with Melissa Liu, Paul Seidel, and Chris Woodward. We would also like to thank Yong-Geun Oh for comments about 
the context of our work and its exposition. Finally, we are grateful to an anonymous referee whose comments have led to significant improvements in the paper.

The work of the first author was supported in part by NSF grant DMS-0600909 and DARPA. The work of the second author was supported in part by NSF grant DMS-0405859. Any opinions, findings and conclusions or recommendations expressed in this material are those of the authors and do not necessarily reflect the views of the National Science Foundation (NSF).

\section{REFERENCES}

[1] P. Albers, "A Lagrangian Piunikhin-Salamon-Schwarz Morphism and Two Comparison Homomorphisms in Floer Homology," math.SG/0512037.

[2] M. Audin, F. Lalonde, L. Polterovich, "Symplectic Rigidity: Lagrangian Submanifolds," in Holomorphic Curves in Symplectic Geometry, Progr. Math 117, Birkhäuser, Basel (1994) 271-321. MR:1274934

[3] E. Bierstone and P. Milman, "Semianalytic and subanalytic sets," Inst. Hautes Études Sci. Publ. Math. 67 (1988), 5-42. MR.972342 (89k:32011)

[4] A. Bondal, "Integrable Systems Related to Triangulated Categories," talk at MSRI workshop on Generalized McKay Correspondences and Representation Theory, 3/21/2006, and private communication regarding collaboration with W.-D. Ruan.

[5] V. Drinfeld, "DG quotients of DG categories," J. Algebra 272 (2004), no. 2, 643-691. MR2028075 (2006e:18018)

[6] A. S. Dubson, "Formule pour l'indice des complexes constructibles et des Modules holonomes," C. R. Acad. Sci. Paris Sér. I Math. 298 (1984), no. 6, 113-116. MR741073 (86e:32016a)

[7] Y. Eliashberg, A. Givental, and H. Hofer, "Introduction to Symplectic Field Theory," GAFA Special Volume, "Vision 2000"; math.SG/0010059. MR1826267 (2002e:53136)

[8] K. Fukaya, "Morse homotopy and its quantization," Geometric topology (Athens, GA, 1993), 409-440, AMS/IP Stud. Adv. Math., 2.1, Amer. Math. Soc., Providence, RI, 1997. MR.1470740 (98i:57061)

[9] K. Fukaya and Y.-G. Oh, "Zero-loop open strings in the cotangent bundle and Morse homotopy," Asian. J. Math. 1 (1997), 96-180. MR1480992 (99e:58038)

[10] K. Fukaya, Y.-G. Oh, H. Ohta, and K. Ono, "Lagrangian Intersection Floer Theory - Anomaly and Obstruction," Kyoto preprint Math 00-17, 2000.

[11] V. Ginsburg, "Characteristic varieties and vanishing cycles," Invent. Math. 84 (1986), 327402. MR833194 (87j:32030)

[12] M. Goresky and R. MacPherson, Stratified Morse Theory. Ergebnisse der Mathematik und ihrer Grenzgebiete (3) 14, Springer-Verlag (1988). MR932724 (90d:57039)

[13] M. Grinberg and R. MacPherson, "Euler characteristics and Lagrangian intersections," in Symplectic geometry and topology (Park City, UT, 1997), 265-293, Amer. Math. Soc., Providence, RI, 1999. MR.1702946 (2000h:32044)

[14] M. Gross and B. Siebert, "Affine manifolds log structures, and mirror symmetry," Turkish J. Math. 27 (2003), 33-60. MR1975331 (2004g:14041)

[15] F. R. Harvey and H. B. Lawson, Jr., "Finite volume flows and Morse theory," Annals of Math. 153, no. 1 (2001), 1-25. MR.1826410 (2002c:58018)

[16] A. Kapustin and E. Witten, "Electric-Magnetic Duality And The Geometric Langlands Program," Commun. Number Theory Phys. 1 (2007), 1-236. MR2306566 (2008g:14018)

[17] M. Kashiwara, "Index theorem for constructible sheaves," Astérisque No. 130 (1985), 193209. MR 804053 (87f:58160)

[18] M. Kashiwara, "Quantization of contact manifolds," Publ. Res. Inst. Math. Sci. 32 (1996), no. 1, 1-7. MR1384750 (96m:58237)

[19] M. Kashiwara and P. Schapira, "Microlocal study of sheaves," Astérisque No. 128 (1985), 235 pp. MR794557(87f:58159)

[20] M. Kashiwara and P. Schapira, Sheaves on manifolds. Grundlehren der Mathematischen Wissenschaften 292, Springer-Verlag (1994). MR 1299726 (95g:58222)

[21] R. Kasturirangan and Y.-G. Oh, "Floer homology of open sets and a refinement of Arnol'd's conjecture," Math. Z. 236 (2001), 151-189. MR1812454 (2001m:53162) 
[22] R. Kasturirangan and Y.-G. Oh, "Quantization of Eilenberg-Steenrod Axioms via Fary Functors," RIMS preprint (1999).

[23] B. Keller, "On the cyclic homology of exact categories," J. Pure Appl. Algebra 136 (1999), no. 1, 1-56. MR.1667558 (99m:18012)

[24] M. Kontsevich, "Deformation quantization of algebraic varieties," Lett. Math. Phys. 56 (2001), no. 3, 271-294. MR 1855264 (2002j:53117)

[25] M. Kontsevich and Y. Soibelman, "Homological Mirror Symmetry and Torus Fibrations," math.SG/0011041.

[26] M. Kontsevich and Y. Soibelman, "Affine Structures and non-Archimedian Analytic Spaces," Progr. Math., vol. 244, Birkhäuser Boston, Boston, MA, 2006, pp. 321-385. MR2181810 (2006j:14054)

[27] V. Lyubashenko, O. Manzyuk, "Quotients of unital $A_{\infty}$-categories," math.CT/0306018.

[28] D. Nadler, "Microlocal branes are constructible sheaves," math.SG/0612399.

[29] S. Piunikhin, D. Salamon, and M. Schwarz, "Symplectic-Floer Donaldson Theory and Quantum Cohomology," Contact and Symplectic Cohomology (Cambridge, 1994), 171-200. Publ. Newton Inst. 8, Cambridge University Press, Cambridge, 1996. MR.1432464 (97m:57053)

[30] P. Polesello and P. Schapira, "Stacks of quantization-deformation modules on complex symplectic manifolds," Int. Math. Res. Not. 2004, no. 49, 2637-2664. MR2077680(2005e:32018)

[31] R. Rouquier, "Categorification of the braid groups," math.RT/0409593.

[32] W. Schmid and K. Vilonen, "Characteristic cycles of constructible sheaves," Invent. Math. 124 (1996), 451-502. MR1369425 (96k:32016)

[33] P. Seidel, Fukaya Categories and Picard-Lefschetz Theory, preprint of book in progress available at math.uchicago.edu/ seidel.

[34] P. Seidel and R. Thomas, "Braid group actions on derived categories of coherent sheaves," Duke Math. J. 108 (2001), no. 1, 37-108. MR.1831820(2002e:14030)

[35] J.-C. Sikorav, "Some properties of holomorphic curves in almost complex manifolds," in Holomorphic Curves in Symplectic Geometry, Birkhäuser (1994), 165-189. MR1274929

[36] L. van den Dries and C. Miller, "Geometric categories and o-minimal structures," Duke Math. J. 84, no. 2 (1996), 497-539. MR1404337 (97i:32008)

Department of Mathematics, Northwestern University, 2033 Sheridan Road, EvansTON, ILLINOIS 60208

E-mail address: nadler@math.northwestern.edu

Department of Mathematics, Northwestern University, 2033 Sheridan Road, EvansTON, ILLINOIS 60208

E-mail address: zaslow@math.northwestern.edu 